

\section{MATHEMATICS AND MECHANICS OF COMPLEX SYSTEMS}

\author{
EDITORIAL BOARD \\ ANTONIO CARCATERRA \\ ERIC A. CARLEN \\ FRANCESCO DELL'ISOLA \\ RAFFAELE ESPOSITO \\ ALbERT FANNJIANG \\ GILLES A. FRANCFORT \\ Pierangelo Marcati \\ Peter A. Markowich \\ MARTIN OSTOJA-STARZEWSKI \\ PiERRE SEPPECHER \\ David J. STEIGMANN \\ Paul Steinmann \\ PIERRE M. SuQueT \\ MANAGING EDITORS \\ MICOL AMAR \\ EMILIO BARCHIESI \\ MARTIN OsTOJA-STARZEWSKI \\ HONORARY EDITORS \\ TEODOR ATANACKOVIĆ \\ VICTOR BERDICHEVSKY \\ GuY BouchitTÉ \\ FELIX DARVE \\ CARlo Marchioro \\ ERRICO PRESUTTI \\ MARIO PULVIRENTI \\ LuCIO Russo \\ ADVISORY BOARD \\ Holm ALTENBACH \\ HARM ASKES \\ ANDREA BRAIDES \\ MAURO CARFORA \\ ERIC DARVE \\ FABRIZIO DAVÌ \\ ANNA DE MASI \\ Emmanuele DiBenedetto \\ VICTOR A. EREMEYEV \\ BERNOLD FIEDLER \\ IRENE M. GAMBA \\ PIERRE GERMAIN \\ SERgey GaVRiLyuk \\ Timothy J. Healey \\ ROBERT P. LIPTON \\ ANGELO LUONGO \\ JUAN J. MANFREDI \\ JEAN-JACQUES MARIGO \\ ANIL MISRA \\ ROBERTO NATALINI \\ Thomas J. Pence \\ ANDREY PIATNITSKI \\ Miguel A. F. SANJUAN \\ A. P. S. Selvadurai \\ MirosLaV ŠILHAVÝ \\ GEORG STADLER \\ Guido SwEERS \\ LEV TRUSKINOVSKY \\ JUAN J. L. VELÁZQUEZ \\ VINCENZO VESPRI \\ VITALY VOLPERT
}

\section{msp.org/memocs}

Università di Roma "La Sapienza", Italia

Rutgers University, USA

(CO-CHAIR) Università di Roma "La Sapienza", Italia

(TREASURER) Università dell'Aquila, Italia

University of California at Davis, USA

(CO-CHAIR) Université Paris-Nord, France

Università dell'Aquila, Italy

DAMTP Cambridge, UK, and University of Vienna, Austria

(CHAIR MANAGING EDITOR) Univ. of Illinois at Urbana-Champaign, USA

Université du Sud Toulon-Var, France

University of California at Berkeley, USA

Universität Erlangen-Nürnberg, Germany

LMA CNRS Marseille, France

Università di Roma "La Sapienza", Italia

Università degli Studi dell'Aquila, Italy

(CHAIR MANAGING EDITOR) Univ. of Illinois at Urbana-Champaign, USA

University of Novi Sad, Serbia

Wayne State University, USA

Université du Sud Toulon-Var, France

Institut Polytechnique de Grenoble, France

Università di Roma "La Sapienza", Italia

Università di Roma Tor Vergata, Italy

Università di Roma "La Sapienza", Italia

Università di Roma "Tor Vergata", Italia

Otto-von-Guericke-Universität Magdeburg, Germany

University of Sheffield, UK

Università di Roma Tor Vergata, Italia

Università di Pavia, Italia

Stanford University, USA

Università Politecnica delle Marche, Ancona (I), Italy

Università dell'Aquila, Italia

Vanderbilt University, USA

Gdansk University of Technology, Poland

Freie Universität Berlin, Germany

University of Texas at Austin, USA

Courant Institute, New York University, USA

Université Aix-Marseille, France

Cornell University, USA

Louisiana State University, USA

Università dell'Aquila, Italia

University of Pittsburgh, USA

École Polytechnique, France

University of Kansas, USA

Istituto per le Applicazioni del Calcolo "M. Picone", Italy

Michigan State University, USA

Narvik University College, Norway, Russia

Universidad Rey Juan Carlos, Madrid, Spain

McGill University, Canada

Academy of Sciences of the Czech Republic

Courant Institute, New York University, United States

Universität zu Köln, Germany

École Polytechnique, France

Bonn University, Germany

Università di Firenze, Italia

CNRS \& Université Lyon 1, France Angelo Vulpiani \& Università di Roma La Sapienza, Italia

MEMOCS (ISSN 2325-3444 electronic, 2326-7186 printed) is a journal of the International Research Center for the Mathematics and Mechanics of Complex Systems at the Università dell'Aquila, Italy.

Cover image: "Tangle" by @ John Horigan; produced using the Context Free program (contextfreeart.org).

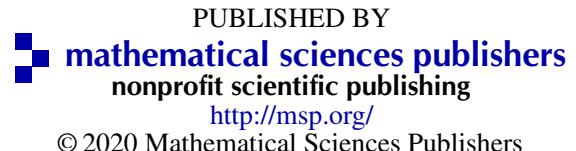

(C) 2020 Mathematical Sciences Publishers 


\title{
ON A STOCHASTIC APPROACH TO MODEL THE DOUBLE PHOSPHORYLATION/DEPHOSPHORYLATION CYCLE
}

\author{
Alberto Maria Bersani, Alessandro Borri, \\ Francesco Carravetta, Gabriella Mavelli and Pasquale Palumbo
}

\begin{abstract}
Because of the unavoidable intrinsic noise affecting biochemical processes, a stochastic approach is usually preferred whenever a deterministic model gives too rough information or, worse, may lead to erroneous qualitative behaviors and/or quantitatively wrong results. In this work we focus on the chemical master equation (CME)-based method which provides an accurate stochastic description of complex biochemical reaction networks in terms of the probability distribution of the underlying chemical populations. Indeed, deterministic models can be dealt with as first-order approximations of the average-value dynamics coming from the stochastic CME approach. Here we investigate the double phosphorylation/dephosphorylation cycle, a well-studied enzymatic reaction network where the inherent double time scale requires one to exploit quasisteady state approximation (QSSA) approaches to infer qualitative and quantitative information. Within the deterministic realm, several researchers have deeply investigated the use of the proper QSSA, agreeing to highlight that only one type of QSSA (the total QSSA) is able to faithfully replicate the qualitative behavior of bistability occurrences, as well as the correct assessment of the equilibrium points, accordingly to the not approximated (full) model. Based on recent results providing CME solutions that do not resort to Monte Carlo simulations, the proposed stochastic approach shows some counterintuitive facts arising when trying to straightforwardly transfer bistability deterministic results into the stochastic realm, and suggests how to handle such cases according to both theoretical and numerical results.
\end{abstract}

\section{Introduction}

One of the main contributions of mathematicians to the biological field is one of the best-known models of enzyme kinetics put forth during the beginning of the twentieth century by Henri [1901a; 1901b; 1902], and Michaelis and Menten [1913],

\section{Communicated by Victor A. Eremeyev.}

MSC2010: primary 34F05, 37L55, 60H10, 60H35; secondary 92C42, 92C45.

Keywords: Michaelis-Menten kinetics, quasisteady state approximation, deterministic and stochastic processes, phosphorylation, chemical master equation, Markov processes. 
and later continued by Briggs and Haldane [1925]. This formulation considers a reaction where a substrate $S$ binds an enzyme $E$ reversibly to form a complex $C$. The complex can then decay irreversibly to a product $P$ and the enzyme, which is then free to bind another molecule of the substrate. These reactions are summarized in the scheme

$$
E+S \underset{k_{-1}}{\stackrel{k_{1}}{\rightleftarrows}} C \stackrel{k_{2}}{\longrightarrow} E+P
$$

where $k_{1}, k_{-1}$, and $k_{2}$ are kinetic parameters associated with the reaction rates (i.e., rate constants).

A very common approximation in the deterministic setting consists of assuming that, after a transient phase, the complex concentration can be considered approximately constant with respect to the substrate dynamics: standard quasisteady state approximation (sQSSA) [Lin and Segel 1988]. The sQSSA utilizes timescale separation to project models of biochemical networks onto lower-dimensional slow manifolds; thus, rapidly fluctuating species are not simulated explicitly (see, among others, [Segel 1988; Segel and Slemrod 1989], and the review paper [Bersani et al. 2015]). In recent decades, many researchers highlighted limits and malfunctioning of the SQSSA, thereby introducing and exploring a new approximation, called total quasisteady state approximation (tQSSA). Under suitable and biologically consistent hypotheses, the tQSSA-based methods were revealed to be very effective in handling the full system of equations, considerably unburdening the computational effort and providing a good approximation at the same time (see, among others, [Laidler 1955; Borghans et al. 1996; Tzafriri 2003; Dell'Acqua and Bersani 2012; Bersani and Dell'Acqua 2012; Bersani et al. 2015]). In the case of reactions that involve only a small number of key regulatory molecules, intrinsic noise is not negligible [Blake et al. 2003; Fedoroff and Fontana 2002], and the enzymatic reaction scheme is more appropriately modeled in a stochastic discrete framework by means of CMEs [van Kampen 2007]. CME-based modeling is a promising approach in systems biology due to its capability of well-fitting experimental data in single-cell experiments, also describing diffusion effects derived from fluctuations and chemical fluxes capable of driving switching from one equilibrium to another. In more detail, the CME provides an accurate stochastic description of complex biochemical networks in terms of the probability distribution of the underlying chemical populations, in contrast to deterministic methods which handle biochemical processes in terms of evolution of the average concentrations for each involved species. Indeed, deterministic models can be dealt with as first-order approximations of the averagevalue dynamics coming from the stochastic CME approach [van Kampen 2007]. Within the framework of enzymatic reaction networks, many authors investigated the QSSAs via the CME approach, with the aim of providing a good approximation of the full model also in this setting [Cao and Petzold 2005; Gillespie 2001; 2009a; 
2009b; Cao et al. 2005; Mastny et al. 2007; Rao and Arkin 2003; Székely and Burrage 2014; Thomas et al. 2011]. In [Barik et al. 2008; MacNamara et al. 2008] the authors studied independently the application of the tQSSA to some well-known biochemical mechanisms providing bistability according to deterministic models.

The work proposed here investigates a CME-based stochastic model of the double phosphorylation/dephosphorylation (PDP) mechanism. This kind of activation/deactivation reaction might be the key to explaining the interactions occurring among the intracellular enzyme networks and several intercellular and macroscopic phenomena, as could be the case highlighted in [George et al. 2019], in the framework of mechanobiology and bone remodeling [Bednarczyk and Lekszycki 2016; Giorgio et al. 2016; 2019; George et al. 2018].

The double PDP cycle is a paradigmatic case of how the application of the sQSSA may provide qualitatively wrong results. With regards to the deterministic approach, several authors (see for example [Ortega et al. 2006; Chickarmane et al. 2007; Kholodenko 2000; Bersani et al. 2011]) studied the appearance of bistable states in the double PDP mechanism, for both the full system and the QSSA settings. In [Dell'Acqua and Bersani 2013; 2011], it is shown that the tQSSA reproduces the behavior of the solutions of the full system for a very wide range of parameters and different initial conditions. On the contrary, the sQSSA can provide misleading results, mainly in the asymptotic concentration values, predicting bistability for large value ranges, whereas the full system (and the tQSSA) shows monostability.

Bistability of several biochemical mechanisms usually in the stochastic framework results in a bimodal stationary probability distribution with randomness allowing for fluctuation around both modes of the distribution, preventing the evolution to stick around just one of the two equilibrium points [Hwang and Velázquez 2013a; 2013b; Bruna et al. 2014; Bazzani et al. 2012; Samoilov et al. 2005]. Within this framework, QSSA still plays an important role to unburden the computational load, though sQSSA may often lead to large errors (both quantitative in matching the wrong modes, and qualitative in failing to catch the bimodal fashion) even when timescale separation holds (see [Kim et al. 2014; 2015] where the stochastic tQSSA is shown to be more accurate than the sQSSA).

Let us underline that references [Kim et al. 2014; 2015] provide very interesting insights into the investigation of stochastic QSS approximations. The common denominator with our work is the way of associating propensities to CME from an ODE initial framework. In more detail, they investigated how different QSS approximations (especially in [Kim et al. 2014], where they consider standard QSSA, total QSSA, and prefactor QSSA) may provide similar results in the deterministic field, but completely different results in the stochastic field. Indeed, the authors showed that, according to a specific setting of the model parameters under investigation, deterministic tQSSA and pQSSA provided the same ODE system 
(therefore leading to the same results), while stochastic tQSSA and pQSSA provided a completely different stochastic approximation. In other words, [Kim et al. 2014] provided some caveat concerning different QSSAs and provided a criterion to understand in a specific framework whether a stochastic QSSA is reliable or not. Unfortunately these results are not straightforwardly applicable to our case: in [Kim et al. 2015] a unique double time scale enzymatic reaction is considered, while in our manuscript we consider four double time scale enzymatic reactions.

Our study investigates the applicability of both standard and total QSSA to a CME-based stochastic model of the double PDP cycle, showing the preeminent role of the tQSSA. The methodology exploited is the one proposed in [Borri et al. 2013; 2016; Bersani et al. 2014] managing to cope with the computational burden, which usually arises for CMEs, by means of a proper organization of the probabilities in the CME entries. This enables us to characterize the CME dynamics according to a recursive block-tridiagonal structure. In this way, explicit solutions of the CME are achieved for both standard and total QSSA, according to a smart application of the Gauss elimination method [Borri et al. 2016]. This allows us not to resort to Monte Carlo simulation, which may be computationally demanding as well as lead to misleading results unless allowing for enough stochastic realizations (whose number is not known a priori).

Preliminary results have been presented in [Bersani et al. 2014], by introducing the CME-based stochastic model for the double PDP cycle. In the present work we get in deeper details, proposing solutions to the CME according to a wider set that goes beyond the toy-setting of [Bersani et al. 2014], facing real numerical problems arising when dealing with the double time scale. Besides the larger variety of cases here reported, we aim at answering the questions arisen in [Bersani et al. 2014] and to conciliate some counterintuitive behaviors occurring when trying to straightforwardly apply deterministic results to stochastic models. To this end, some new theoretical results on the CME-based stochastic model have been assessed, showing the uniqueness of the stationary probability distribution.

The result of the work is twofold. On the one hand, it shows the preeminent role of the tQSSA, also in the stochastic CME-based model of the double PDP cycle. Its ability to faithfully replicate in the stochastic framework the qualitative behavior of bistability occurrences is shown, as well as the correct assessment of the equilibrium points, in conformity with the nonapproximated model. On the other hand, according to a given setting in the parameter space, and to chosen initial conditions, bistability provided by the deterministic model may be lost in a unimodal distribution when dealing with the stochastic CME. Such a mode coincides with one of the two deterministic equilibria. This counterintuitive result is obtained by means of Monte Carlo simulation for the full system, and is confirmed by the exact CME solution provided by the tQSSA. 
The paper is organized as follows. In Section 2 we briefly recall the most important background concerning the sQSSA and the tQSSA for enzymatic reactions. Section 3 proposes the double PDP reactions in detail, dealing with the CME-based stochastic model. Section 4 reports the standard and total QSSA of the double PDP cycle CME-based stochastic model, by first providing them in the deterministic framework. A novel result on the uniqueness of the stationary probability distribution is also provided in this section. In Section 5 we discuss the appearance (or the absence) of stationary bimodality in comparison to bistability arising in deterministic models. Section 6 contains the concluding remarks and perspectives.

\section{Introductory notions on SQSSA and tQSSA}

The Michaelis-Menten (MM) kinetics give a very good description of (1), in terms of ordinary differential equations (ODEs). For notational convenience we will use the same symbol to denote both a chemical species and its concentration (i.e., the variables of the ODEs), omitting its dependence on time. We can mathematically describe reaction (1) using the mass action principle - where the growth rates of each reactant are proportional to the instantaneous concentrations of the reactants themselves - and conservation laws. This approach leads to the (full) system

$$
\left\{\begin{array}{l}
\frac{d S}{d t}=-k_{1}\left(E_{T}-C\right) S+k_{-1} C, \\
\frac{d C}{d t}=k_{1}\left(E_{T}-C\right) S-\left(k_{-1}+k_{2}\right) C,
\end{array}\right.
$$

with the initial conditions

$$
S(0)=S_{T}, \quad C(0)=0, \quad E(0)=E_{T}
$$

and the conservation laws

$$
E+C=E_{T}, \quad S+C+P=S_{T},
$$

where $E_{T}$ and $S_{T}$ are the total enzyme and substrate concentrations, respectively.

The MM reaction is characterized by two phases: a short transient phase of rapid increase of the complex $C$ and a second, slower, phase, called the quasisteady state phase, where the complex is considered substantially in equilibrium.

The hypothesis of quasisteady state simplifies the reaction, leading to an ODE for the substrate, with initial condition $S(0)=S_{T}$, while the complex is assumed to be in a quasisteady state, i.e., $\frac{d C}{d t} \approx 0$. The standard QSSA (sQSSA) of system (2) is thus achieved:

$$
\left\{\begin{aligned}
C & \approx E_{T} \cdot S /\left(K_{M}+S\right), \\
\frac{d S}{d t} & \approx-k_{2} C \approx-V_{\max } S /\left(K_{M}+S\right), \\
S(0) & =S_{T},
\end{aligned}\right.
$$


where $K_{M}=\left(k_{2}+k_{-1}\right) / k_{1}$ is called the MM constant or affinity constant, and $V_{\max }=k_{2} E_{T}$.

Let us consider again the classical MM kinetics (2). Introducing the total substrate at generic time instant $t, \bar{S}(t)=S(t)+C(t)$, (2) then becomes

$$
\left\{\begin{aligned}
\frac{d \bar{S}}{d t} & =-k_{2} C, \\
\frac{d C}{d t} & =k_{1}\left[C^{2}-\left(E_{T}+\bar{S}+K_{M}\right) C+E_{T} \bar{S}\right], \\
\bar{S}(0) & =S_{T}, C(0)=0,
\end{aligned}\right.
$$

with conservation laws

$$
E+C=E_{T}, \quad \bar{S}+P=S_{T} .
$$

Assuming that the complex is in a quasisteady state $\left(\frac{d C}{d t} \approx 0\right)$ yields the total QSSA (tQSSA) [Borghans et al. 1996], which is valid for a broader range of parameters, with respect to sQSSA, covering both high and low enzyme concentrations:

$$
\frac{d \bar{S}}{d t} \approx-k_{2} C_{-}(\bar{S}), \quad \bar{S}(0)=S_{T},
$$

where

$$
C_{-}(\bar{S})=\frac{\left(E_{T}+K_{M}+\bar{S}\right)-\sqrt{\left(E_{T}+K_{M}+\bar{S}\right)^{2}-4 E_{T} \bar{S}}}{2}
$$

is the only biologically allowed solution of $\frac{d C}{d t}=0$ in the second equation of (6).

\section{CME-based stochastic model of the double PDP cycle}

The double PDP cycle is one of the most important biochemical mechanisms in intracellular reaction networks. The scheme here investigated is a generalization of the enzymatic reaction network (1) and refers to [Ortega et al. 2006], where both phosphorylation and dephosphorylation are supposed to happen in only one step. Reactions are reported in (10), where $M, M_{p}$, and $M_{p p}$ represent the inactive, the mono-phosphorylated, and the double-phosphorylated substrate, respectively, $K$ and $P$ are the kinase (the phosphorylating enzyme) and the phosphatase (the dephosphorylating enzyme), respectively, and $C_{i}$ are the intermediate complexes:

$$
\begin{array}{cc}
M+K \underset{k_{-11}}{\stackrel{k_{11}}{\longrightarrow}} C_{1} \stackrel{k_{12}}{\longrightarrow} M_{p}+K, & M_{p}+K \underset{k_{-21}}{\stackrel{k_{21}}{\longrightarrow}} C_{2} \stackrel{k_{22}}{\longrightarrow} M_{p p}+K, \\
M_{p p}+P \underset{k_{-31}}{\stackrel{k_{31}}{\longrightarrow}} C_{3} \stackrel{k_{32}}{\longrightarrow} M_{p}+P, & M_{p}+P \underset{k_{-41}}{\stackrel{k_{41}}{\longrightarrow}} C_{4} \stackrel{k_{42}}{\longrightarrow} M+P .
\end{array}
$$

Before building the stochastic model, we write the deterministic full system by exploiting the mass conservation law to reduce the system complexity. Indeed, the 
conservation law involves the total substrate $M_{T}$,

$$
M+M_{p}+M_{p p}+C_{1}+C_{2}+C_{3}+C_{4}=M_{T},
$$

and the total enzymes $K_{T}$ and $P_{T}$,

$$
K+C_{1}+C_{2}=K_{T}, \quad P+C_{3}+C_{4}=P_{T},
$$

so that it is possible to reduce the number of deterministic variables (species concentrations) to six independent ones. By taking, for example, $\left\{M, M_{p p}, C_{1}, C_{2}, C_{3}, C_{4}\right\}$ as the set of the independent variables, using the law of mass action, the full system of equations governing the dynamics of the system is therefore

$$
\begin{aligned}
\frac{d M}{d t} & =-k_{11} M K+k_{-11} C_{1}+k_{42} C_{4}, \\
\frac{d M_{p p}}{d t} & =-k_{31} M_{p p} P+k_{-31} C_{3}+k_{22} C_{2}, \\
\frac{d C_{1}}{d t} & =k_{11} M K-\left(k_{-11}+k_{12}\right) C_{1}, \\
\frac{d C_{2}}{d t} & =k_{21} M_{p} K-\left(k_{-21}+k_{22}\right) C_{2}, \\
\frac{d C_{3}}{d t} & =k_{31} M_{p p} P-\left(k_{-31}+k_{32}\right) C_{3}, \\
\frac{d C_{4}}{d t} & =k_{41} M_{p} P-\left(k_{-41}+k_{42}\right) C_{4},
\end{aligned}
$$

with initial conditions

$$
M(0)=M_{T}, \quad M_{p p}(0)=0, \quad C_{i}(0)=0,
$$

where $i=1, \ldots, 4$. Let us observe that, for the sake of brevity, we left in (13) the terms $M_{p}, K$, and $P$, which are related to the six independent variables by (11) and (12):

$$
\begin{gathered}
M_{p}=M_{T}-\left(M_{p p}+C_{1}+C_{2}+C_{3}+C_{4}+M\right), \\
K=K_{T}-\left(C_{1}+C_{2}\right), \quad P=P_{T}-\left(C_{3}+C_{4}\right) .
\end{gathered}
$$

According to a large variety of literature that manages to reformulate the dynamics of a system from an ODE into a CME (chemical master equation) framework (see, e.g., [Bazzani et al. 2012] or [Bersani et al. 2014]), we treat the state variables as discrete copy numbers. In this context, we reinterpret the deterministic reaction rates as probabilities per unit time (or propensities) of a properly defined continuous-time Markov chain (CTMC), i.e., a stochastic process whose 


\begin{tabular}{|l|cc|}
\hline \multicolumn{1}{|c|}{ event } & reset & propensity \\
\hline$M, K$ binding & $x_{1} \mapsto x_{1}-1$ & $k_{11} x_{1}\left(K_{T}-x_{3}-x_{4}\right)$ \\
$M, K$ unbinding & $x_{3} \mapsto x_{3}+1$ & \\
& $x_{1} \mapsto x_{1}+1$ & $k_{-11} x_{3}$ \\
$M_{p}$ release & $x_{3} \mapsto x_{3}-1$ & $k_{12} x_{3}$ \\
$M_{p}, K$ binding & $x_{3} \mapsto x_{3}-1$ & $\left.x_{4}^{6} x_{i}\right)\left(K_{T}-x_{3}-x_{4}\right)$ \\
$M_{p}, K$ unbinding & $x_{4} \mapsto x_{4}+1$ & $k_{21}\left(M_{T}-\sum_{4}-1\right.$ \\
$M_{p p}$ release & $x_{2} \mapsto x_{2}+1$ & $k_{-21} x_{4}$ \\
& $x_{4} \mapsto x_{4}-1$ & $k_{22} x_{4}$ \\
$M_{p p}, P$ binding & $x_{2} \mapsto x_{2}-1$ & $k_{31} x_{2}\left(P_{T}-x_{5}-x_{6}\right)$ \\
& $x_{5} \mapsto x_{5}+1$ & \\
$M_{p p}, P$ unbinding & $x_{2} \mapsto x_{2}+1$ & $k_{-31} x_{5}$ \\
$M_{p}$ release & $x_{5} \mapsto x_{5}-1$ & \\
$M_{p}, P$ binding & $x_{5} \mapsto x_{5}-1$ & $k_{32} x_{5}$ \\
$M_{p}, P$ unbinding & $x_{6} \mapsto x_{6}+1$ & $x_{41}\left(M_{T}-\sum_{i=1}^{6} x_{i}\right)\left(P_{T}-x_{5}-x_{6}\right)$ \\
$M$ release & $x_{1} \mapsto x_{1}+1$ & $k_{-41} x_{6}$ \\
& $x_{6} \mapsto x_{6}-1$ & $k_{42} x_{6}$ \\
& & \\
\hline
\end{tabular}

Table 1. Chemical reactions, full system.

trajectories evolve on an $n$-dimensional lattice, and whose dynamics (in terms of probability of being in a specific state of the CTMC) is described by the CME.

Renaming the independent state variables in the CME-based stochasting setting as $x_{1}=M, x_{2}=M_{p p}, x_{3}=C_{1}, x_{4}=C_{2}, x_{5}=C_{3}$, and $x_{6}=C_{4}$, the reset map associated with the chemical reaction network in (10) is reported in Table 1.

The CME dynamics is $\dot{P}=G \mathscr{P}$, where $G$ is called the infinitesimal generator (or transition matrix) of the CTMC, which is built according to the propensities in Table 1 (see [Borri et al. 2016] for further details), and $\mathscr{P}$ is the vector collecting the time-varying probabilities of all the states of the process. When the dimension of $G$ is large enough to make computationally too demanding the exact solution of the equilibrium equation, $G \mathscr{P}=0$, one can still employ the Gillespie stochastic simulation algorithm (SSA) [Gillespie 1977; 2001; 2009a; 2009b; Cao et al. 2005], returning one or more statistically correct trajectories of the process, which can be used in a Monte Carlo simulation or in an ergodic setting to obtain a sampled (approximate) equilibrium distribution of the process. 


\section{QSSA of the double PDP cycle stochastic model}

Similarly to the deterministic approach, where the complexes behave as fast variables, while the substrates are the slow variables, the double time scale affects as well the CMEs associated with the reset map detailed in Table 1, slowing down the computational efficiency. Therefore, a need exists to introduce a QSSA also for the stochastic case. Differently from other reactions, as for example the so-called auxiliary (or coupled) reactions [Eilertsen and Schnell 2018], where multiple timescales are present, the four reactions involved in the PDP cycle are characterized by a double timescale. In [Bersani et al. 2014] a way to obtain both standard and total QSSA for the double PDP cycle was shown. In both cases, we start from the ODE version of the QSSA and stochastify it by introducing suitably defined one-step processes. Note that, as already described at the end of Section 3, the stochastic approach considered in [Bersani et al. 2014] and here does not consist of perturbing the ODE setting by means of noise terms, which is typical of the stochastic differential equation (SDE)/Langevin approach, but of reinterpreting the deterministic reaction rates as probabilities per unit time (or propensities) of a properly defined CTMC.

With respect to the ODE systems reported below, the continuous state variables actually represent a first-order approximation of the expected value of the copy numbers, which are indeed stochastic variables [van Kampen 2007]. Section 4.1 treats the QSSAs of the ODE system, whereas Section 4.2 concerns the CME-based stochastic version of the QSSAs. Finally, in Section 4.3 a sufficient condition for the uniqueness of the stationary solution for the CME has been provided.

4.1. QSSA of the deterministic model of the double PDP cycle. With regard to the SQSSA, its ODE version is written by setting the complex dynamics at steady state. In this way complexes are related to substrate and enzyme concentrations by means of algebraic constraints and, after computations (see [Bersani et al. 2011] and references therein for the details), the $M$ and $M_{p p}$ dynamics become

$$
\begin{aligned}
\frac{d M}{d t} & =-\frac{k_{12}}{K_{1}} M K+\frac{k_{42}}{K_{4}} M_{p} P, \\
\frac{d M_{p p}}{d t} & =\frac{k_{22}}{K_{2}} M_{p} K-\frac{k_{32}}{K_{3}} M_{p p} P,
\end{aligned}
$$

where $K_{i}=\left(k_{-i 1}+k_{i 2}\right) / k_{i 1}, i=1, \ldots, 4$, with

$$
K=\frac{K_{T}}{1+M / K_{1}+M_{p} / K_{2}}, \quad P=\frac{P_{T}}{1+M_{p p} / K_{3}+M_{p} / K_{4}} .
$$

Concerning $M_{p}$, the sQSSA constrains it to other substrates according to

$$
M_{p}=M_{T}-M-M_{p p},
$$

thus neglecting the complexes' contribution to the mass conservation law. 
Remark. The comparison of the mass conservation law of the full system (11) with (17) leads to the so-called complex depletion paradox [Dell'Acqua and Bersani 2013]: the application of the sQSSA implies that, even if the complexes are related to the substrates by their algebraic equations, they are implicitly set equal to zero, because of (17). The consequences are that the sQSSA predicts asymptotic values for the different substrate species which are higher than those predicted by the full system.

For what concerns the tQSSA, as in [Bersani et al. 2011; Dell'Acqua and Bersani $2013 ; 2011]$, we set the total substrates at a generic time instant $t$ :

$$
\bar{M}=M+C_{1}, \quad \bar{M}_{p}=M_{p}+C_{2}+C_{4}, \quad \bar{M}_{p p}=M_{p p}+C_{3} .
$$

In terms of these new variables, the dynamics of the total substrates are given by

$$
\begin{gathered}
\frac{d \bar{M}}{d t}=k_{42} C_{4}-k_{12} C_{1}, \\
\frac{d \bar{M}_{p p}}{d t}=-k_{32} C_{3}+k_{22} C_{2},
\end{gathered}
$$

with conservation law

$$
\bar{M}+\bar{M}_{p}+\bar{M}_{p p}=M_{T}
$$

Moreover, by properly accounting for the QSSA, i.e., complexes' dynamics at steady state, we have the constraints

$$
\begin{aligned}
\left(\bar{M}-C_{1}\right)\left(K_{T}-C_{1}-C_{2}\right)-K_{1} C_{1} & =0, \\
\left(\bar{M}_{p}-C_{2}-C_{4}\right)\left(K_{T}-C_{1}-C_{2}\right)-K_{2} C_{2} & =0, \\
\left(\bar{M}_{p p}-C_{3}\right)\left(P_{T}-C_{3}-C_{4}\right)-K_{3} C_{3} & =0, \\
\left(\bar{M}_{p}-C_{2}-C_{4}\right)\left(P_{T}-C_{3}-C_{4}\right)-K_{4} C_{4} & =0 .
\end{aligned}
$$

Remark. It is worth noting that the complex depletion paradox emerging for the sQSSA is not present for the total, since no violation of the mass conservation law occurs. Thus, the tQSSA yields the same asymptotic values for all the reactants (complexes included) as the full system.

4.2. QSSA of the CME-based stochastic model of the PDP cycle. In agreement with [Bazzani et al. 2012] or [Bersani et al. 2014], we treat the state variables in (15) and (19) as discrete copy numbers that increase by one or decrease by one (according to a one-step process approach [van Kampen 2007]), with propensity provided by the sum of the production or clearance rates of the ODE dynamics for the one-step increase or decrease reaction, respectively. In this way, the reset maps associated with the standard and total QSSA are reported in Tables 2 and 3, 


\begin{tabular}{|l|cc|}
\hline \multicolumn{1}{|c|}{ event } & reset & propensity \\
\hline$M$ production & $M \mapsto M+1$ & $w_{1}^{(s)}$ \\
$M$ clearance & $M \mapsto M-1$ & $w_{2}^{(s)}$ \\
$M_{p p}$ production & $M_{p p} \mapsto M_{p p}+1$ & $w_{3}^{(s)}$ \\
$M_{p p}$ clearance & $M_{p p} \mapsto M_{p p}-1$ & $w_{4}^{(s)}$ \\
\hline
\end{tabular}

Table 2. Chemical reactions, sQSSA.

\begin{tabular}{|l|cc|}
\hline \multicolumn{1}{|c|}{ event } & reset & propensity \\
\hline $\bar{M}$ production & $\bar{M} \mapsto \bar{M}+1$ & $w_{1}^{(t)}$ \\
$\bar{M}$ clearance & $\bar{M} \mapsto \bar{M}-1$ & $w_{2}^{(t)}$ \\
$\bar{M}_{p p}$ production & $\bar{M}_{p p} \mapsto \bar{M}_{p p}+1$ & $w_{3}^{(t)}$ \\
$\bar{M}_{p p}$ clearance & $\bar{M}_{p p} \mapsto \bar{M}_{p p}-1$ & $w_{4}^{(t)}$ \\
\hline
\end{tabular}

Table 3. Chemical reactions, tQSSA.

respectively. Regarding the propensities, the ones of the sQSSA, achieved from (15), by exploiting constraints (16) and (17), are

$$
w_{j}^{(s)}\left(x_{1}, x_{2}\right)= \begin{cases}\frac{k_{42} P_{T} K_{3}\left(M_{T}-x_{1}-x_{2}\right)}{K_{3} K_{4}+K_{4} x_{2}+\left(M_{T}-x_{1}-x_{2}\right) K_{3}}, & j=1, \\ \frac{k_{12} K_{T} K_{2} x_{1}}{K_{1} K_{2}+K_{2} x_{1}+K_{1}\left(M_{T}-x_{1}-x_{2}\right)}, & j=2, \\ \frac{k_{22} K_{T} K_{1}\left(M_{T}-x_{1}-x_{2}\right)}{K_{1} K_{2}+K_{2} x_{1}+K_{1}\left(M_{T}-x_{1}-x_{2}\right)}, & j=3, \\ \frac{k_{32} P_{T} K_{4} x_{2}}{K_{3} K_{4}+K_{4} x_{2}+K_{3}\left(M_{T}-x_{1}-x_{2}\right)}, & j=4 .\end{cases}
$$

With regards to the tQSSA, one needs to solve the system of equations (21) with respect to the complexes $C_{1}, \ldots, C_{4}$ (see, e.g., [Pedersen et al. 2008]), which are functions of the state, and then define

$$
w_{j}^{(t)}\left(x_{1}, x_{2}\right)= \begin{cases}k_{42} C_{4}\left(x_{1}, x_{2}\right), & j=1, \\ k_{12} C_{1}\left(x_{1}, x_{2}\right), & j=2, \\ k_{22} C_{2}\left(x_{1}, x_{2}\right), & j=3, \\ k_{32} C_{3}\left(x_{1}, x_{2}\right), & j=4 .\end{cases}
$$

4.3. Uniqueness of the stationary distribution. An important feature to be investigated when dealing with stochastic models coming from CME is whether the stationary distribution is unique, whatever the CME initial conditions. Besides the qualitative behavior properties, the uniqueness of the stationary distribution is 
invoked also when dealing with Monte Carlo numerical issues, since it implies the process is ergodic, thus allowing one to resort to a unique very long run of the stochastic sampling algorithm (SSA), by inferring the statistical distribution from the computation of the average recurrence time in each state of the process. With respect to the PDP cycle investigated in this paper, such an issue is of paramount importance: in case of bistability, what one is expected to find from the CME stochastic approach is to have a bimodal stationary distribution, with the modes close to the ODE stable stationary equilibria. However, supposing that bimodality actually occurs, it may happen that any single stochastic realization is able to exhibit only one of the two modes, according to the chosen initial conditions. This is because the model parameters are such that too much time would be required in order to completely explore the space of the admissible states. To know a priori whether the stationary distribution is unique or not allows one to understand if different stochastic realizations provide different stationary distributions because of different initial conditions or just because of the too long time required to obtain a stochastically exhaustive trajectory. To properly address the issue concerning the uniqueness of the stationary distribution, we consider the graph associated with the CTMC of the CME under investigation.

\section{Theorem 1. The graph associated with the full system is strongly connected.}

Proof. The graph associated with the full system consists of as many nodes as feasible 6-tuples provided by the copy numbers of the independent species $M, M_{p p}$, $C_{1}, C_{2}, C_{3}, C_{4}$, with node $A$ connected to node $B$ if there exists a reaction that updates the species' copy numbers from $A$ to $B$. Differently from a one-step process, here we have reactions that simultaneously vary couples of state variables, namely $M, K$ binding and unbinding, $M_{p p}$ release, $M_{p p}, P$ binding and unbinding, and $M$ release (see Table 1). The proof consists of showing that, starting from any 6-tuple, there exists a combination of feasible reactions providing any possible onestep update. This fact allows the system to inherit the strong connectivity property associated with one-step processes. The one-step updates we consider, clearly, disregard the ones already provided by the chemical reaction network. Below, for any such one-step update we report the sequence of reactions required to obtain it:

- $M \mapsto M+1$ : provided by the combination of $M_{p}, P$ binding and $M$ release.

- $M \mapsto M-1$ : provided by the combination of $M, K$ binding and $M_{p}$ release.

- $M_{p p} \mapsto M_{p p}+1$ : provided by the combination of $M_{p}, K$ binding and $M_{p p}$ release.

- $M_{p p} \mapsto M_{p p}-1$ : provided by the combination of $M_{p p}, P$ binding and $M_{p}$ release. 
- $C_{1} \mapsto C_{1}+1$ : provided by the combination of $M, K$ binding, $M_{p}, P$ binding, and $M$ release.

- $C_{3} \mapsto C_{3}+1$ : provided by the combination of $M_{p}, K$ binding, $M_{p p}, P$ binding, and $M_{p p}$ release.

Theorem 1 proves that the graph associated with the CME here considered is strongly connected, i.e., for any two nodes of the graph there exists a path that connects one node to the other, and vice versa. This is sufficient to prove that there exists a unique terminal strongly connected component associated with any of the three graphs (the graph itself, actually), and this proves the uniqueness of the stationary solution for the CME [Bang-Jensen and Gutin 2009].

Remark. To prove the strong connectivity of both the QSSAs is trivial. Indeed, they are built as one-step processes; therefore, the graph associated with them is a complete 2D lattice where any point is reachable from any other by means of one-step independent movement. Therefore, results provided in Section 3 for the full system, dealing with the uniqueness of the stationary probability distribution, hold true also for both standard and total QSSA.

\section{ODE bistability versus CME bimodality: a word of caution}

This section is devoted to investigating and discussing whether bistability behavior arising from deterministic models of the double PDP cycle transforms into a bimodal distribution of the stationary probability distribution coming from the CME-based stochastic model. To this end, Monte Carlo simulations are carried out when dealing with the original full system, while numerical tools providing the CME analytical solution are exploited for both standard and total QSSAs.

In the following four illustrated cases, the values of the chosen parameters in (10) are $k_{11}=0.02, k_{-11}=1, k_{12}=0.01 ; k_{21}=0.032, k_{-21}=1, k_{22}=15 ; k_{31}=0.045$, $k_{-31}=1, k_{32}=0.092$; and $k_{41}=0.01, k_{-41}=1, k_{42}=0.5$.

We set $M_{T}=500$ and four different pairs of mass-balance constraints for the kinase and phosphatase:

(a) $K_{T}=200, P_{T}=200$,

(b) $K_{T}=600, P_{T}=400$,

(c) $K_{T}=292, P_{T}=300$,

(d) $K_{T}=293, P_{T}=300$,

with initial condition $M(0)=M_{T}$ in the deterministic case. Following [Dell'Acqua and Bersani 2011] (see Figures 3 and 4 therein), when we plot the initial value of the kinase MAPKK, i.e., $K_{T}$ (on the horizontal axis) and the corresponding asymptotic value of $M_{p p}$ (on the vertical axis), we obtain: 
(a) The deterministic full system is bistable, with equilibrium points for $M_{p p}$ equal to

$$
M_{p p}^{1}=4.30, \quad M_{p p}^{2}=290.97 ;
$$

the deterministic sQSSA and tQSSA are able to recover the bistable behavior, but with completely different levels of accuracy. In fact, the equilibria are

$$
M_{p p}^{s, 1}=2.52, \quad M_{p p}^{s, 2}=494.35
$$

for the sQSSA, and

$$
M_{p p}^{t, 1}=4.31, \quad M_{p p}^{t, 2}=290.86
$$

for the tQSSA. This implies that also in this case the tQSSA is much more reliable than the sQSSA, which in fact suffers from the so-called complex depletion paradox, as discussed in [Dell'Acqua and Bersani 2011].

(b) The deterministic full system is monostable, with equilibrium point for $M_{p p}$ equal to

$$
M_{p p}=118.89 ;
$$

the deterministic tQSSA is able to reproduce this feature:

$$
M_{p p}^{t}=118.17 .
$$

As discussed in [Dell'Acqua and Bersani 2013; 2011], the sQSSA always gives bistability in a larger set of values of $K_{T}$ than the full system and the tQSSA; in fact, in this case the sQSSA has two stable states

$$
M_{p p}^{s, 1}=9.08, \quad M_{p p}^{s, 2}=496.94,
$$

which are very far from the real equilibrium.

(c) The full system is bistable, with equilibrium points for $M_{p p}$ equal to

$$
M_{p p}^{1}=5.70, \quad M_{p p}^{2}=185 ;
$$

the deterministic sQSSA and tQSSA are able to reproduce the bistable behavior, but with completely different levels of accuracy. In fact, the equilibria are

$$
M_{p p}^{s, 1}=2.30, \quad M_{p p}^{s, 2}=494
$$

for the sQSSA, and

$$
M_{p p}^{t, 1}=5.70, \quad M_{p p}^{t, 2}=185
$$

for the tQSSA. This case also confirms the superiority of the tQSSA with respect to the sQSSA. 
(d) This case presents the same features as case (c), but even if it slightly differs in the amount of the mass-balance constraint $K_{T}$ for the kinase, it is of interest because of the peculiar phenomenon shown by its stochastic counterpart, as described below.

We now compare the behavior of the stochastic representations of full system as well as the sQSSA and tQSSA. With regards to the full system, the state evolves on a 6-dimensional lattice (copy numbers refer to $M, M_{p p}$ and the 4 complexes), formally bounded by the substrate upper bound $\left(M_{T}=500\right)$ and the complexes' upper bounds provided by $\min \left\{K_{T}, M_{T}\right\}$ for $C_{1}, C_{2}$ and $\min \left\{P_{T}, M_{T}\right\}$ for $C_{3}, C_{4}$. For instance, concerning case (a), the lattice is inside a 6-dimensional box lattice including $500^{2} \times 200^{4} \simeq 400$ trillion states. Clearly, not all such states are admissible (e.g., because they can violate the mass conservation laws) but, in any case, there still remain too many states (billions) that prevent any reliable numerical approach to straightforwardly solve the underlining CME. For this reason, the full system is simulated by means of statistical methods, such as the Gillespie stochastic simulation algorithm (SSA) [Gillespie 1977; 2001; 2009a; 2009b; Cao et al. 2005], where Theorem 1 ensures the uniqueness of the stationary probability distribution and the ergodicity of the CTMC associated with the CME.

On the other hand, both the standard and total QSSA evolve on a (lower) 2dimensional lattice (copy numbers refer to substrates $M, M_{p p}$ or total substrates $\bar{M}, \bar{M}_{p p}$, respectively), formally bounded by the 2 -dimensional box lattice including $500 \times 500=250000$ states, that reduce to about 125000 when accounting for mass conservation laws. These numbers allow one to compute explicitly, and in a computationally very efficient way, the exact theoretical distribution by applying Gaussian elimination, or block-based efficient solvers [Borri et al. 2016], to the CME equilibrium problem. The numerical simulations were performed in the Matlab suite on an Apple MacBook Pro laptop with $2.5 \mathrm{GHz}$ Intel Core i5 CPU and 16 GB RAM. The computation time of the equilibrium distribution is just 3 seconds for the SQSSA and for the tQSSA.

Figure 1 shows the plot of the steady-state marginal distribution of species $M_{p p}$ for case (a). It is apparent that both the standard and total QSSA resemble the full system, although the stochastic modes are not able to catch both equilibrium points coming from the deterministic approach, since apparent unimodal distributions come out. Indeed, the modes of the three distributions substantially reproduce the lower equilibrium point, with the full system and the total QSSA providing a slightly better match than the standard QSSA.

Figure 2 reports the steady-state marginal distribution of species $M_{p p}$ for case (b). Again, we have a very good match between full system and tQSSA, both providing a unimodal distribution, with the mode resembling the unique equilibrium point of 


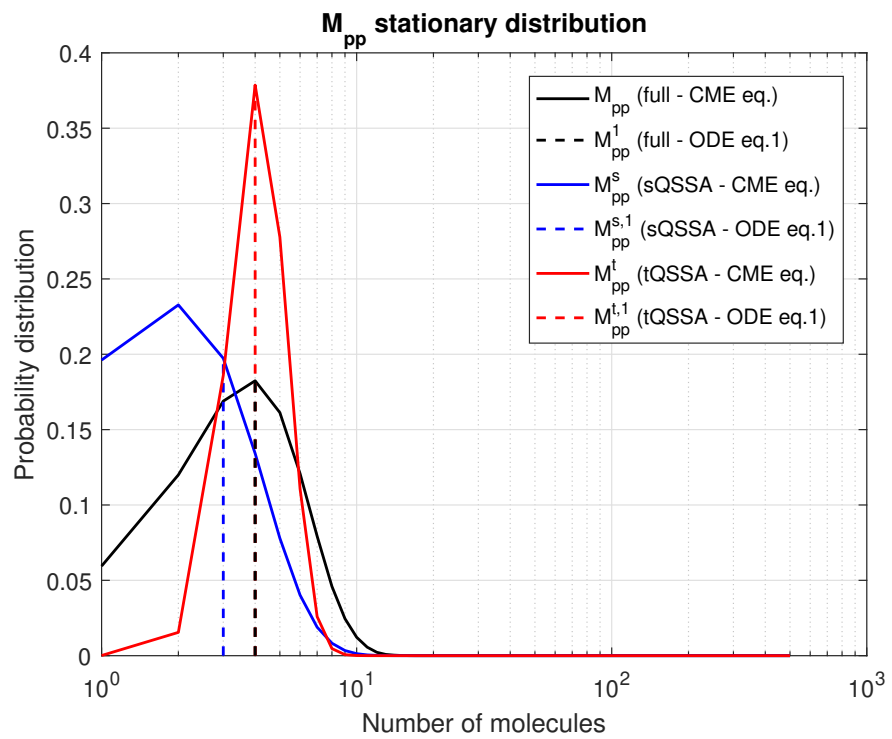

Figure 1. Stochastic setting of the case (a). Steady-state marginal distribution of species $M_{p p}$. The blue solid line represents the steady-state distribution computed by means of the Gillespie algorithm for the full model of reactions, while the black and red solid lines represent the sQSSA and tQSSA distributions, respectively. The deterministic equilibria are reported in dashed lines. The plot shows that the second modes, which are present in the deterministic counterpart, are not detected. The system appears to be monostable.

the deterministic model; the stochastic sQSSA also exhibits one mode, corresponding to the higher equilibrium of its deterministic counterpart and is hence very far from the modes of full system and tQSSA.

Finally, Figures 3 and 4 show the steady-state marginal distribution of species $M_{p p}$ for cases (c) and (d). Again it is apparent that the Monte Carlo simulation of the full system confirms that only the tQSSA provides a very good approximation. Case (c), for instance, shows that the tQSSA (as well as the full system) provides a bimodal distribution, with the modes corresponding to the equilibrium points of the deterministic model, while the sQSSA provides a unimodal distribution. Moreover, by slightly varying the parameter setting of just 1 copy number, case (d) shows a completely different qualitative behavior, with full and tQSSA providing a unimodal distribution (with the mode resembling the highest of the 2 equilibrium points of the deterministic model), while the sQSSA provides a unimodal distribution completely different from the one coming from the full system. 


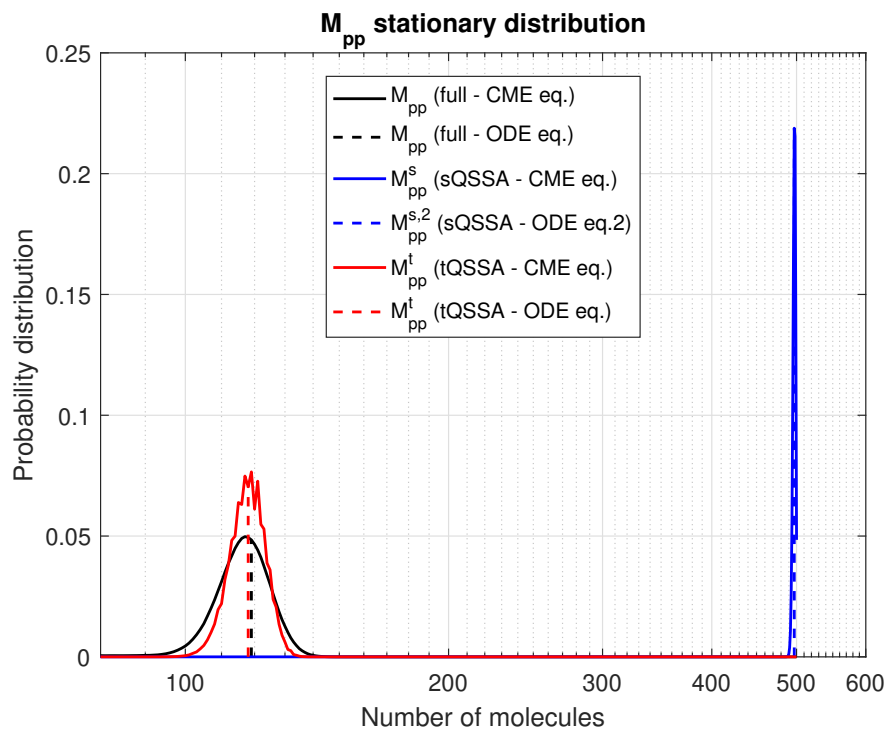

Figure 2. Stochastic setting of the case (b). Steady-state marginal distribution of species $M_{p p}$. The blue solid line represents the steady-state distribution computed by means of the Gillespie algorithm for the full system of reactions, while the black and red solid lines represent the sQSSA and tQSSA distributions, respectively. The deterministic equilibria are reported in dashed lines. Differently from sQSSA, the tQSSA reproduces with very good approximation the mode of the full system.

One important consideration that comes out from these results is that the stochastic tQSSA seems to be a promising tool for the approximation of the distribution of the full system, when the computation of the exact equilibrium distribution of this system is intractable. Note also that the dimensions of resulting CME matrices are exactly the same in SQSSA and tQSSA, so there is no loss in computational performance in exploiting the latter, which is far more accurate than SQSSA in capturing the position of the modes. Indeed, results from Theorem 1 allow one to trust the stationary distribution as coming from different initial conditions as the unique one, and the correctness of the full system stationary distribution allows one to trust the tQSSA (instead of the SQSSA) as the golden standard to approximate (at least) the steady-state behavior. This result somehow mimics what is already known from the deterministic viewpoint.

Another important consideration concerns the topic of mono/bistability. It is thus important to note that a bistable behavior in the deterministic approach (i.e., in the ODEs) is not (necessarily) associated with a bimodal behavior in the stochastic 


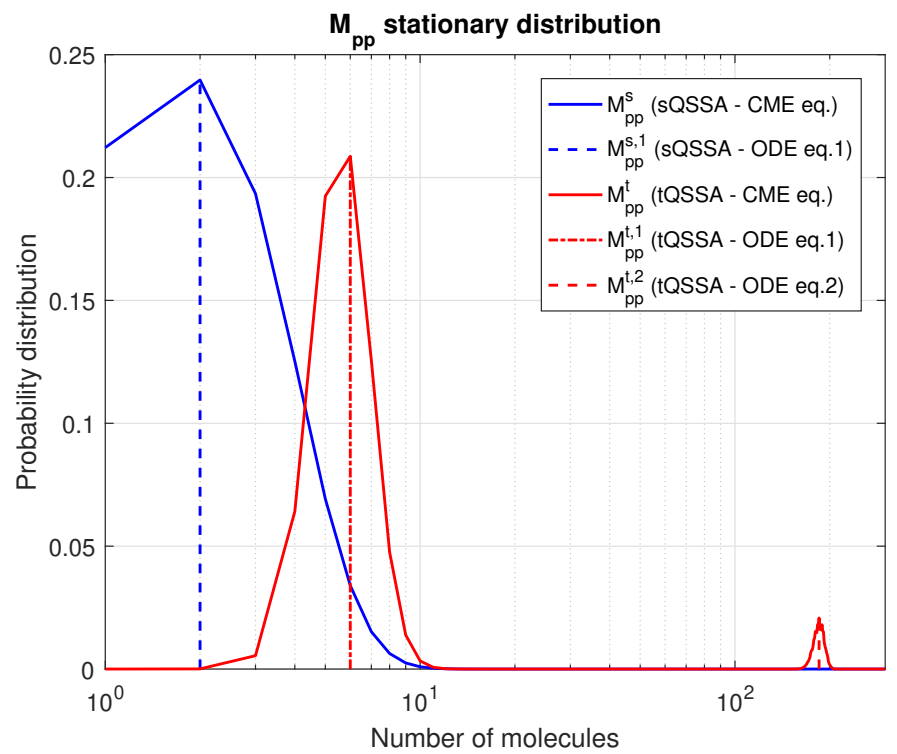

Figure 3. Stochastic setting of the case (c). Steady-state marginal distribution of species $M_{p p}$. The blue and red solid lines represent the sQSSA and tQSSA distributions, respectively. The deterministic equilibria are reported in dashed lines. The tQSSA reproduces with very good approximation the bistable behavior of the full system, whereas the sQSSA shows a monostable behavior, contrary to what happens in the deterministic setting.

approach (i.e., in the CMEs). Indeed, among the analyzed cases, in cases (a) and (d) numerical simulations show that the stochastic setting presents just one mode, matching one of the two deterministic equilibria. A possible explanation for this phenomenon is that when we consider a deterministic system, we can study its basins of attraction, from which the trajectories flow necessarily towards the same equilibrium point. On the other hand, in the stochastic framework, a trajectory (realization) can always go from any state to any other one during the evolution of the system, in that the graph of reactions is connected and the Markov model is positively recurrent (see [Borri et al. 2016] for further details). So, it is reasonable that, depending on the propensity values, one of the two deterministic equilibrium points can be visited much more often than the other one and that the trajectories (almost) never leave a neighborhood of the dominating point, which is stochastically a kind of black hole. As a consequence, the other equilibrium point disappears from the plots of the probability distribution and the stochastic system behavior is qualitatively monomodal. Indeed, there is still a way to recover (at least numerically) the bimodal behavior which is not present at a stochastic macroscopic 


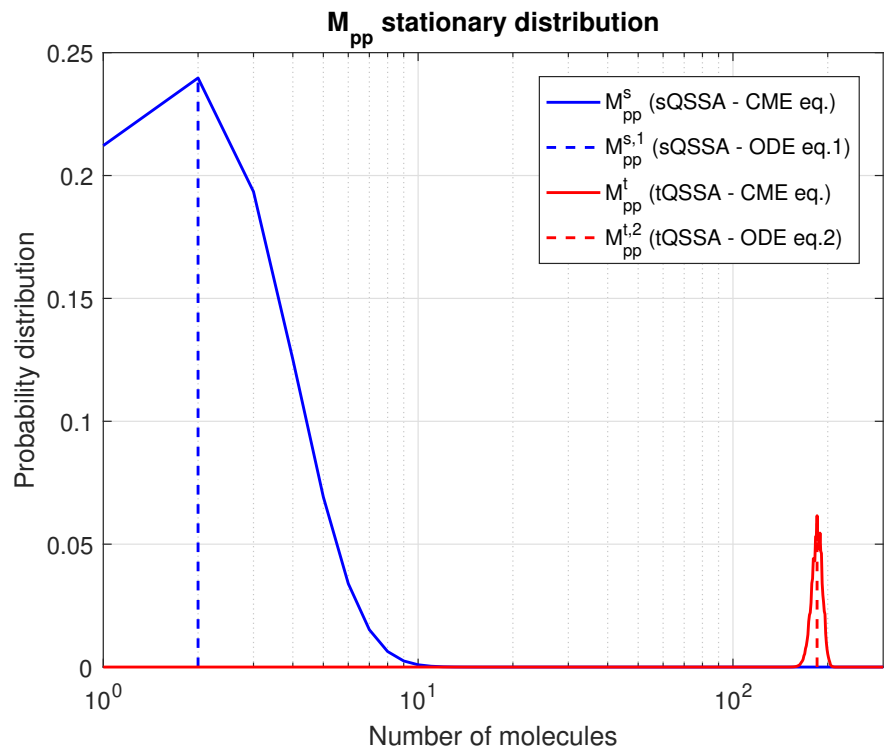

Figure 4. Stochastic setting of the case (d). Steady-state marginal distribution of species $M_{p p}$. The blue and red solid lines represent the SQSSA and tQSSA distributions, respectively. The deterministic equilibria are reported in dashed lines. Differently from the deterministic setting, both the stochastic QSSAs present only one mode, but while the tQSSA exactly reproduces the second mode of the full system, the sQSSA wrongly reproduces the mode.

level. For the case (a), a plot of the discrete derivative of the steady-state marginal distribution of the species $M_{p p}$, for sQSSA and tQSSA, is shown in Figure 5, and it shows that both SQSSA and tQSSA exhibit some zero crossings of the derivative corresponding to the second deterministic equilibrium point (around $M_{p p}=495$ in sQSSA and $M_{p p}=290$ in tQSSA), which are necessary conditions for the existence of second modes. Anyway, such modes are not detected in Figure 1.

In conclusion, the simulations show the deeper insight of the stochastic approach into the understanding of the qualitative behavior of reaction networks; in particular, stochastic simulations are able to provide information about the actual probability of reaching different equilibrium conditions. This information is lost in the deterministic approach which constitutes just a first-order approximation of the mean value of the CME [van Kampen 2007]. Based on this statement, we can assert that a mode of the stochastic approach always has an equilibrium point as its deterministic counterpart, but that the converse is not always true. Furthermore, in both settings, the superiority of the tQSSA approach compared to the sQSSA is confirmed, in that the former shows a much greater numerical accuracy than 

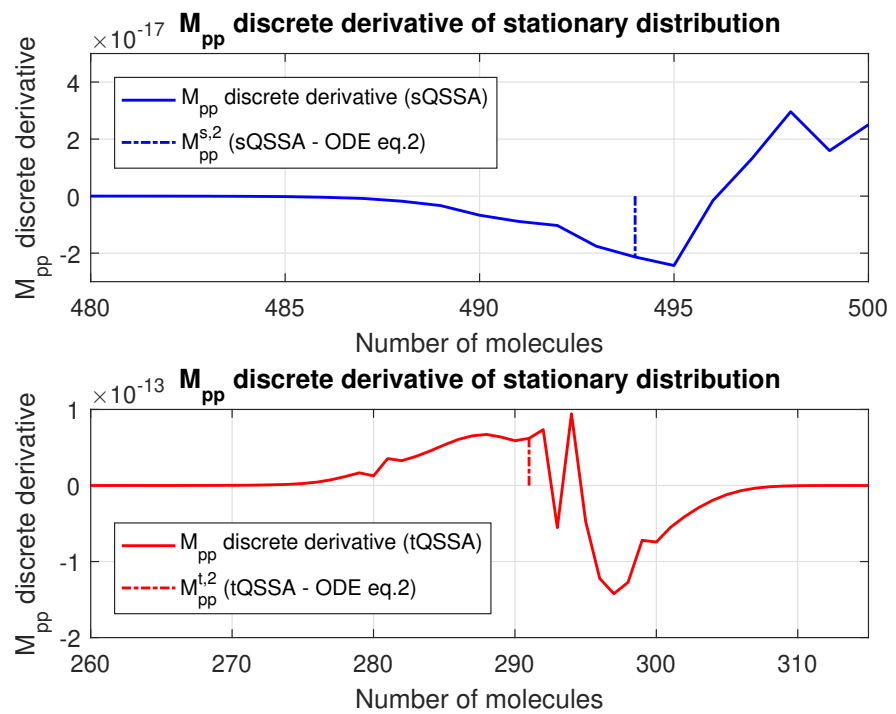

Figure 5. Discrete derivative of the steady-state marginal distribution of species $M_{p p}$ (stochastic case), in case (a), for the sQSSA (top) and tQSSA (bottom) distributions, respectively. The plots show the existence of the second mode (zero crossing of the discrete derivative) in the zone of the second deterministic equilibrium point (reported in dashed line), both in SQSSA and in tQSSA.

the latter in matching the equilibrium points/modes. In the stochastic approach, in addition, the better performance of tQSSA is obtained at the same computational cost as in sQSSA, since the obtained CME dynamical matrices have the same dimension in the two cases.

\section{Conclusions}

In this work we investigated a stochastic approach for modeling the biochemical reaction cycle of double phosphorylation/dephosphorylation (PDP), which is one of the most important biochemical mechanisms in intracellular reaction networks. The goal of the work is twofold. On the one hand, we aimed to understand whether the QSSA approach could be transferred to the investigation of the qualitative behavior of the double PDP cycle also in the stochastic scheme, usually assessed as the best approach whenever dealing with biochemical processes which are intrinsically noisy and for which the average copy number dynamics is the only (and little informative) result available from a deterministic approach. Our results have somehow extended to the stochastic realm results already established from the deterministic approach: the tQSSA is a superior tool (with respect to sQSSA) to 
deal with affordable approximations, since it is able to faithfully replicate the full system results also in those cases where sQSSA fails. On the other hand, proposed results show how deterministic models could produce misleading results if not properly accounted for in the wider setting of the stochastic approach, according to which ODE models can be thought of as a first-order approximation of the average dynamics. Indeed, what emerges is that, in some cases, deterministic bistability does not provide a stationary bimodal distribution.

It is reasonable to state that the apparently paradoxical phenomenon described above may be explained by the following considerations. Continuous-time Markov chains (CTMCs) describe the probabilities for the state of a discrete-event system to stay in a specific point on the state space. According to a given reaction, the dynamics of these probabilities are described by the CME, with reaction rates providing the propensities for the state to jump from one point of the space to another one. We therefore conjecture that the disappearance of one mode in the stationary bimodal distribution could occur when the probability of leaving one basin of attraction is so low that it would require a (quite) infinite time to occur. In this case, we can expect a switching phenomenon from one state to the other one after a very long time, in contrast with what occurs in the deterministic case, as observed, in a different context, in [Székely and Burrage 2014]. Under the hypothesis that the ergodicity property of CTMC holds, it is well-known that the stationary probability distribution is unique whatever the initial condition, and that statistical properties can be deduced from a single, sufficiently long realization (stochastic realization) of the stochastic process. The fact that this feature of Markov chains obviously cannot be captured by the deterministic approach could be the reason for the discrepancy between the two different approaches. Waiting for one single (long enough) Gillespie stochastic simulation, instead of running a (large enough) number of them, could be a way to capture the second stable state of a bistable system. The aim of our future work will be to give further explanations of this phenomenon which is still a subject of our studies.

Finally, as already observed in the introduction, in [Kim et al. 2014] it was investigated how different QSS approximations (standard QSSA, total QSSA, and prefactor QSSA) may provide similar results in the deterministic field, while providing completely different results in the stochastic field. In other words, [Kim et al. 2014] provided some caveat concerning different QSSAs and provided a criterion to understand in a specific framework whether a stochastic QSSA is reliable or not. Unfortunately these results are not straightforwardly applicable to our case: in [Kim et al. 2015] a unique double time scale enzymatic reaction is considered, while in our manuscript we consider four double time scale enzymatic reactions. However, we plan to extend such results to our more general framework in the continuation of our research. 


\section{References}

[Bang-Jensen and Gutin 2009] J. Bang-Jensen and G. Gutin, Digraphs: theory, algorithms and applications, 2nd ed., Springer, 2009.

[Barik et al. 2008] D. Barik, M. R. Paul, W. T. Baumann, Y. Cao, and J. J. Tyson, "Stochastic simulation of enzyme-catalyzed reactions with disparate timescales", Biophys. J. 95:8 (2008), 35633574 .

[Bazzani et al. 2012] A. Bazzani, G. C. Castellani, E. Giampieri, D. Remondini, and L. N. Cooper, "Bistability in the chemical master equation for dual phosphorylation cycles", J. Chem. Phys. 136:23 (2012), art. id. 235102.

[Bednarczyk and Lekszycki 2016] E. Bednarczyk and T. Lekszycki, "A novel mathematical model for growth of capillaries and nutrient supply with application to prediction of osteophyte onset", $Z$. Angew. Math. Phys. 67:4 (2016), art. id. 94.

[Bersani and Dell'Acqua 2012] A. M. Bersani and G. Dell'Acqua, "Is there anything left to say on enzyme kinetic constants and quasi-steady state approximation?", J. Math. Chem. 50:2 (2012), $335-344$.

[Bersani et al. 2011] A. M. Bersani, G. Dell'Acqua, and G. Tomassetti, "On stationary states in the double phosphorylation-dephosphorylation cycle", pp. 1208-1211 in Proceedings of the International Conference on Numerical Analysis and Applied Mathematics 2011 (Halkidiki, Greece, 2011), AIP Conference Proceedings 1389, American Institute of Physics, College Park, MD, 2011.

[Bersani et al. 2014] A. M. Bersani, A. Borri, F. Carravetta, G. Mavelli, and P. Palumbo, "Quasisteady-state approximations of the chemical master equation in enzyme kinetics: application to the double phosphorylation/dephosphorylation cycle", pp. 3053-3058 in 53rd IEEE Conference on Decision and Control (Los Angeles, 2014), IEEE, Piscataway, NJ, 2014.

[Bersani et al. 2015] A. M. Bersani, E. Bersani, G. Dell'Acqua, and M. G. Pedersen, "New trends and perspectives in nonlinear intracellular dynamics: one century from Michaelis-Menten paper", Contin. Mech. Thermodyn. 27:4-5 (2015), 659-684.

[Blake et al. 2003] W. J. Blake, M. Kærn, C. R. Cantor, and J. J. Collins, "Noise in eukaryotic gene expression”, Nature 422 (2003), 633-637.

[Borghans et al. 1996] J. A. M. Borghans, R. J. de Boer, and L. A. Segel, "Extending the quasi-steady state approximation by changing variables”, B. Math. Biol. 58:1 (1996), 43-63.

[Borri et al. 2013] A. Borri, F. Carravetta, G. Mavelli, and P. Palumbo, "Some results on the structural properties and the solution of the chemical master equation", pp. 3771-3776 in 2013 American Control Conference (Washington, DC, 2013), IEEE, Piscataway, NJ, 2013.

[Borri et al. 2016] A. Borri, F. Carravetta, G. Mavelli, and P. Palumbo, "Block-tridiagonal statespace realization of chemical master equations: a tool to compute explicit solutions", J. Comput. Appl. Math. 296 (2016), 410-426.

[Briggs and Haldane 1925] G. E. Briggs and J. B. S. Haldane, "A note on the kinetics of enzyme action", Biochem. J. 19:2 (1925), 338-339.

[Bruna et al. 2014] M. Bruna, S. J. Chapman, and M. J. Smith, "Model reduction for slow-fast stochastic systems with metastable behaviour", J. Chem. Phys. 140:17 (2014), art. id. 174107.

[Cao and Petzold 2005] D. T. Cao, Yang Gillespie and L. R. Petzold, "The slow-scale stochastic simulation algorithm", J. Chem. Phys. 122:1 (2005), art. id. 014116.

[Cao et al. 2005] Y. Cao, D. Gillespie, and L. Petzold, "Multiscale stochastic simulation algorithm with stochastic partial equilibrium assumption for chemically reacting systems", J. Comput. Phys. 206:2 (2005), 395-411. 
[Chickarmane et al. 2007] V. Chickarmane, B. N. Kholodenko, and H. M. Sauro, "Oscillatory dynamics arising from competitive inhibition and multisite phosphorylation", J. Theoret. Biol. 244:1 (2007), 68-76.

[Dell'Acqua and Bersani 2011] G. Dell'Acqua and A. Bersani, "Bistability and the complex depletion paradox in the double phosphorylation-dephosphorylation cycle", pp. 55-56 in Proceedings of the International Conference on Bioinformatics Models, Methods and Algorithms (Rome, 2011), vol. 1: Bioinformatics, SciTePress, Setúbal, Portugal, 2011.

[Dell'Acqua and Bersani 2012] G. Dell'Acqua and A. M. Bersani, "A perturbation solution of Michaelis-Menten kinetics in a "total" framework", J. Math. Chem. 50:5 (2012), 1136-1148.

[Dell'Acqua and Bersani 2013] G. Dell'Acqua and A. M. Bersani, "Quasi-steady state approximations and multistability in the double phosphorylation-dephosphorylation cycle", pp. 155-172 in Biomedical Engineering Systems and Technologies, Communications in Computer and Information Science 273, Springer, 2013.

[Eilertsen and Schnell 2018] J. Eilertsen and S. Schnell, "A kinetic analysis of coupled (or auxiliary) enzyme reactions", B. Math. Biol. 80:12 (2018), 3154-3183.

[Fedoroff and Fontana 2002] N. Fedoroff and W. Fontana, "Small numbers of big molecules", Science 297:5584 (2002), 1129-1131.

[George et al. 2018] D. George, R. Allena, and Y. Rémond, "Cell nutriments and motility for mechanobiological bone remodeling in the context of orthodontic periodontal ligament deformation", J. Cell. Immunoth. 4:1 (2018), 26-29.

[George et al. 2019] D. George, R. Allena, and Y. Rémond, "Integrating molecular and cellular kinetics into a coupled continuum mechanobiological stimulus for bone reconstruction", Contin. Mech. Thermodyn. 31:3 (2019), 725-740.

[Gillespie 1977] D. T. Gillespie, "Exact stochastic simulation of coupled chemical reactions", $J$. Chem. Phys. 81:25 (1977), 2340-2361.

[Gillespie 2001] D. T. Gillespie, "Approximate accelerated stochastic simulation of chemically reacting systems”, J. Chem. Phys. 115:4 (2001), 1716-1733.

[Gillespie 2009a] D. T. Gillespie, "Deterministic limit of stochastic chemical kinetics", J. Chem. Phys. B 113:6 (2009), 1640-1644.

[Gillespie 2009b] D. T. Gillespie, "A diffusional bimolecular propensity function", J. Chem. Phys. 131:16 (2009), art. id. 164109.

[Giorgio et al. 2016] I. Giorgio, U. Andreaus, D. Scerrato, and F. dell'Isola, "A visco-poroelastic model of functional adaptation in bones reconstructed with bio-resorbable materials", Biomech. Model. Mechan. 15:5 (2016), 1325-1343.

[Giorgio et al. 2019] I. Giorgio, F. dell'Isola, U. Andreaus, F. Alzahrani, T. Hayat, and T. Lekszycki, "On mechanically driven biological stimulus for bone remodeling as a diffusive phenomenon", Biomech. Model. Mechan. 18:6 (2019), 1639-1663.

[Henri 1901a] V. Henri, "Recherches sur la loi de l'action de la sucrase", C. R. Hebd. Acad. Sci. 133 (1901), 891-899.

[Henri 1901b] V. Henri, “Über das Gesetz der Wirkung des Invertins”, Z. Phys. Chem. 39 (1901), 194-196.

[Henri 1902] V. Henri, “Théorie générale de l'action de quelques diastases", C. R. Hebd. Acad. Sci. 135 (1902), 916-919.

[Hwang and Velázquez 2013a] H. J. Hwang and J. J. L. Velázquez, "Bistable stochastic biochemical networks: highly specific systems with few chemicals", J. Math. Chem. 51:5 (2013), 1343-1375. 
[Hwang and Velázquez 2013b] H. J. Hwang and J. J. L. Velázquez, "Bistable stochastic biochemical networks: large chemical networks and systems with many molecules", J. Math. Chem. 51:8 (2013), 2074-2103.

[van Kampen 2007] N. G. van Kampen, Stochastic processes in physics and chemistry, 3rd ed., Lecture Notes in Mathematics 888, North-Holland, Amsterdam, 2007.

[Kholodenko 2000] B. N. Kholodenko, "Negative feedback and ultrasensitivity can bring about oscillations in the mitogen-activated protein kinase cascades", Eur. J. Biochem. 267:6 (2000), 15831588 .

[Kim et al. 2014] J. K. Kim, K. Josić, and M. R. Bennett, "The validity of quasi-steady-state approximations in discrete stochastic simulations", Biophys. J. 107:3 (2014), 783-793.

[Kim et al. 2015] J. K. Kim, K. Josić, and M. R. Bennett, "The relationship between stochastic and deterministic quasi-steady state approximations”, BMC Syst. Biol. 9 (2015), art. id. 87.

[Laidler 1955] K. J. Laidler, "Theory of the transient phase in kinetics, with special reference to enzyme systems", Can. J. Chem. 33:10 (1955), 1614-1624.

[Lin and Segel 1988] C. C. Lin and L. A. Segel, Mathematics applied to deterministic problems in the natural sciences, 2nd ed., Classics in Applied Mathematics 1, Society for Industrial and Applied Mathematics, Philadelphia, 1988.

[MacNamara et al. 2008] S. MacNamara, A. M. Bersani, K. Burrage, and R. B. Sidje, "Stochastic chemical kinetics and the total quasi-steady-state assumption: application to the stochastic simulation algorithm and chemical master equation”, J. Chem. Phys. 129:9 (2008), art. id. 095105.

[Mastny et al. 2007] E. A. Mastny, E. L. Haseltine, and J. B. Rawlings, "Two classes of quasi-steadystate model reductions for stochastic kinetics", J. Chem. Phys. 127:9 (2007), art. id. 094106.

[Michaelis and Menten 1913] L. Michaelis and M. L. Menten, "Die Kinetik der Invertinwirkung”, Biochem. Z. 49 (1913), 333-369.

[Ortega et al. 2006] F. Ortega, J. L. Garcés, F. Mas, B. N. Kholodenko, and M. Cascante, "Bistability from double phosphorylation in signal transduction: kinetic and structural requirements", $F E B S J$. 273:17 (2006), 3915-3926.

[Pedersen et al. 2008] M. G. Pedersen, A. M. Bersani, E. Bersani, and G. Cortese, "The total quasisteady-state approximation for complex enzyme reactions", Math. Comput. Simulation 79:4 (2008), 1010-1019.

[Rao and Arkin 2003] C. V. Rao and A. P. Arkin, "Stochastic chemical kinetics and the quasi-steadystate assumption: application to the Gillespie algorithm", J. Chem. Phys. 118:11 (2003), 49995010.

[Samoilov et al. 2005] M. Samoilov, S. Plyasunov, and A. P. Arkin, "Stochastic amplification and signaling in enzymatic futile cycles through noise-induced bistability with oscillations", $P$. Natl. Acad. Sci. USA 102:7 (2005), 2310-2315.

[Segel 1988] L. A. Segel, "On the validity of the steady state assumption of enzyme kinetics", $B$. Math. Biol. 50:6 (1988), 579-593.

[Segel and Slemrod 1989] L. A. Segel and M. Slemrod, "The quasi-steady-state assumption: a case study in perturbation”, SIAM Rev. 31:3 (1989), 446-477.

[Székely and Burrage 2014] T. Székely, Jr. and K. Burrage, "Stochastic simulation in systems biology", Comput. Struct. Biotech. J. 12:20-21 (2014), 14-25.

[Thomas et al. 2011] P. Thomas, A. V. Straube, and R. Grima, "Communication: limitations of the stochastic quasi-steady-state approximation in open biochemical reaction networks", J. Chem. Phys. 135: 18 (2011), art. id. 181103.

[Tzafriri 2003] A. R. Tzafriri, "Michaelis-Menten kinetics at high enzyme concentrations", B. Math. Biol. 65:6 (2003), 1111-1129. 
Received 10 Nov 2019. Revised 24 Jun 2020. Accepted 28 Jul 2020.

ALBERTO MARIA BERSANI: alberto.bersani@uniroma1.it

Dipartimento di Ingegneria Meccanica e Aerospaziale, Università degli Studi di Roma "La Sapienza", Rome, Italy

ALESSANDRO BORRI: alessandro.borri@iasi.cnr.it

Istituto di Analisi dei Sistemi et Informatica "Antonio Ruberti”, Consiglio Nazionale delle Ricerche, Rome, Italy

FRANCESCO CARRAVETTA: francesco.carravetta@iasi.cnr.it

Istituto di Analisi dei Sistemi et Informatica "Antonio Ruberti”, Consiglio Nazionale delle Ricerche, Rome, Italy

GABRIELLA MAVELLI: gabriella.mavelli@iasi.cnr.it

Istituto di Analisi dei Sistemi et Informatica "Antonio Ruberti”, Consiglio Nazionale delle Ricerche, Rome, Italy

PASQUale PALUmbo: pasquale.palumbo@unimib.it

Dipartimento di Biotecnologie e Bioscienze, Università degli Studi Milano-Bicocca, Milano, Italy

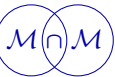





\title{
A NEW COMPREHENSIVE APPROACH FOR BONE REMODELING UNDER MEDIUM AND HIGH MECHANICAL LOAD BASED ON CELLULAR ACTIVITY
}

\author{
Daniel George, Rachele Allena, Céline Bourzac, Stéphane Pallu, \\ Morad Bensidhoum, Hugues Portier AND Yves RÉmond
}

\begin{abstract}
Most of the last century, bone remodeling models have been proposed based on the observation that bone density is dependent on the intensity of the applied mechanical loads. Most of these cortical or trabecular bone remodeling models are related to the osteocyte mechanosensitivity, and they all have a direct correlation between the bone mineral density and the mechanical strain energy. However, experiments on human athletes show that high-intensity sport activity tends not to increase bone mineral density but rather has a negative impact. Therefore, it appears that the optimum bone mineral density would develop for "medium"-intensity activity (or medium mechanical loads) and not for the highest-intensity one.

In this work, we propose a new continuum approach based on bone cell activity being either positive or negative as a function of the intensity of the applied mechanical load. At standard earth gravity without exercise, bone homeostasis is observed with cell activity being at equilibrium. When "medium loads" such as "low-intensity" or "optimized" sport activity are applied, cells are activated and an increase of bone density occurs. On the other hand, "high-intensity loads" such as over-training lead to bone density decrease or bone degradation. Our results are in agreement with the literature and enable us to foresee applications such as optimal sport training for best physical conditions.
\end{abstract}

\section{Introduction}

The last hundred years or so have seen many bone remodeling models being developed under the hypothesis that the mechanical energy is the main driving parameter of this complex phenomenon. According to the first law of bone remodeling defined in [Wolff 1986] and reprinted many times, bone mineral density is directly

\section{Communicated by Emilio Barchiesi.}

George and Allena share first-authorship.

MSC2010: 65Z05, 92C05.

Keywords: bone remodeling, cellular activity, high and medium mechanical loads, osteoblasts, osteoclasts. 
dependent on the intensity of the applied mechanical loads. Many strain-related models for bone remodeling have followed since. To name a few, see for example [Carter 1984], [Frost 1987] and its "mechanostat" proposal, [Cowin 1986], [Beaupré et al. 1990], [Turner 1998], or more recently [Lekszycki 2002] where the highly heterogeneous bone microstructure is seen as an optimum structural response to given external mechanical boundary conditions. Many theoretical frameworks of bone remodeling were proposed for cortical [Pivonka et al. 2008] and trabecular [Ruimerman et al. 2005] bone accounting for different bone cells at the origin of this remodeling [Pivonka and Komarova 2010; Klein-Nulend et al. 2013]. More recently, further theoretical models were proposed [Madeo et al. 2011; 2012; Lekszycki and dell' Isola 2012; Scala et al. 2017], some of them also taking into account the complex viscous mechanical behavior of the bone [Andreaus et al. 2014b; Giorgio et al. 2016; 2017].

Nowadays, it is generally accepted that without considering the specific effects of the bone cells, whatever the theoretical model, the prediction of bone remodeling remains at best phenomenologically driven. Although at the continuous level (scale of the bone) the continuum mechanics is "manageable", the integration of continuum biology is highly risky since, for the time being, there are no experimental measurements available in the literature able to link the local cell phenomena to the bone continuum. The full understanding of the bone mechanobiology is still unknown, but some literature exists on its basic principles (see for example [Burr and Allen 2014, pp. 85-86]). It is therefore possible to start developing more precise mechanobiological models, but at the local scale (scale of the cells), and to try understanding what the main biological parameters driving this evolution are [Lemaire et al. 2011]. Nonetheless, bridging the local and the global scales through multiscale or homogenization models remains a challenging task [Lemaire et al. 2005; 2010; 2015]. Multiscale theories on biological materials have been developed recently (see, e.g., [Rémond et al. 2016; George et al. 2017; Spingarn et al. 2017]), but the uncertainties remain about the multiscale models themselves [Sansalone et al. 2015], the scale growth response [Louna et al. 2016], or even the influence of the microstructure on the overall behavior [Sheidaei et al. 2019].

The obvious next step in bone remodeling is to try integrating more biological actions that are at the origin of the bone tissue evolution such as the capillary growth [Bednarczyk and Lekszycki 2016], the nutrient supply [Lu and Lekszycki 2016], or the cell migration [Allena and Maini 2014; Schmitt et al. 2015; Frame et al. 2019; 2018]. However, the homogenization of such local effects at more macroscopic scales [George et al. 2018a; 2018b; 2019] is complex to transpose and interpret. This is even without accounting for thermodynamically consistent models [Martin et al. 2017] or the influence of the bone microstructure distribution over its macroscopic behavior [Bagherian et al. 2019]. 
At this stage, it is clear that bone mechanobiology is a highly complex phenomenon and, even with the major progress made in the past fifty years, we still know very little to properly describe the bone remodeling scenario. In this work, we want to address the bone remodeling phenomenon at a macroscopic scale based on the direct relationship between the mechanical strain and the bone cell responses (see, e.g., [Ehrlich and Lanyon 2002]). Our approach is evidently dependent on the activation of osteoblasts for the bone creation and of the osteoclasts for the bone resorption triggered by specific signals that are transmitted by the osteocytes, as proposed in [Ignatius et al. 2005; Andreaus et al. 2014a; Rochefort et al. 2010]. In agreement with the experimental literature [Herman et al. 2010; Hao et al. 2017], we find that bone mineral density does not continually increase with the developed mechanical strain, contrarily to the proposed "mechanostat" model by Frost [1987], and that reverse effects can be observed.

\section{Model development}

2.1. Model construction. We propose a strain energy density (SED) based approach accounting for external mechanical loads driving the cell activity and leading to a macroscopic bone mineral density change at the continuous scale.

It has been observed that practicing a regular physical activity is healthy not only for the heart, but also to reinforce bone structure and stiffness. However, it was also acknowledged that over-training could lead to a worse health condition than the moderate training scenario [Forwood and Parker 1989; Grimston et al. 1991; Herman et al. 2010; Hao et al. 2017]. Thus, for "medium" mechanical loads (i.e., intermittent sport activity under a certain threshold), the bone mineral density increases, whereas for "high" mechanical loads (i.e., critical above the given threshold), the bone mineral density decreases.

We want to keep a simplified approach in order to be able to identify the model's parameters and to validate it. Our main assumption is that the cell activity is directly proportional to the intensity of the mechanical load, but with a predefined scenario. We define such a cell activity as the quantity of bone formation/resorption (or bone mineral density change) as a function of time and mechanical energy. Hence, for a given mechanical energy level, the cell activity will change the bone mineral density in a given time. The cell activity increases with the mechanical load up to a certain value that is proportional to the amount of available space within the structure (related to the porosity and defined in Figure 1 by the maximum osteoblast activity per unit volume). The cell activity cannot exceed this value and a maximum cellular density exists. The maximum cellular activity (of osteoblasts or osteoclasts) is dependent on the cell density at a given location and at a given time. It is assumed that this activity linearly increases (with the mechanical load) 


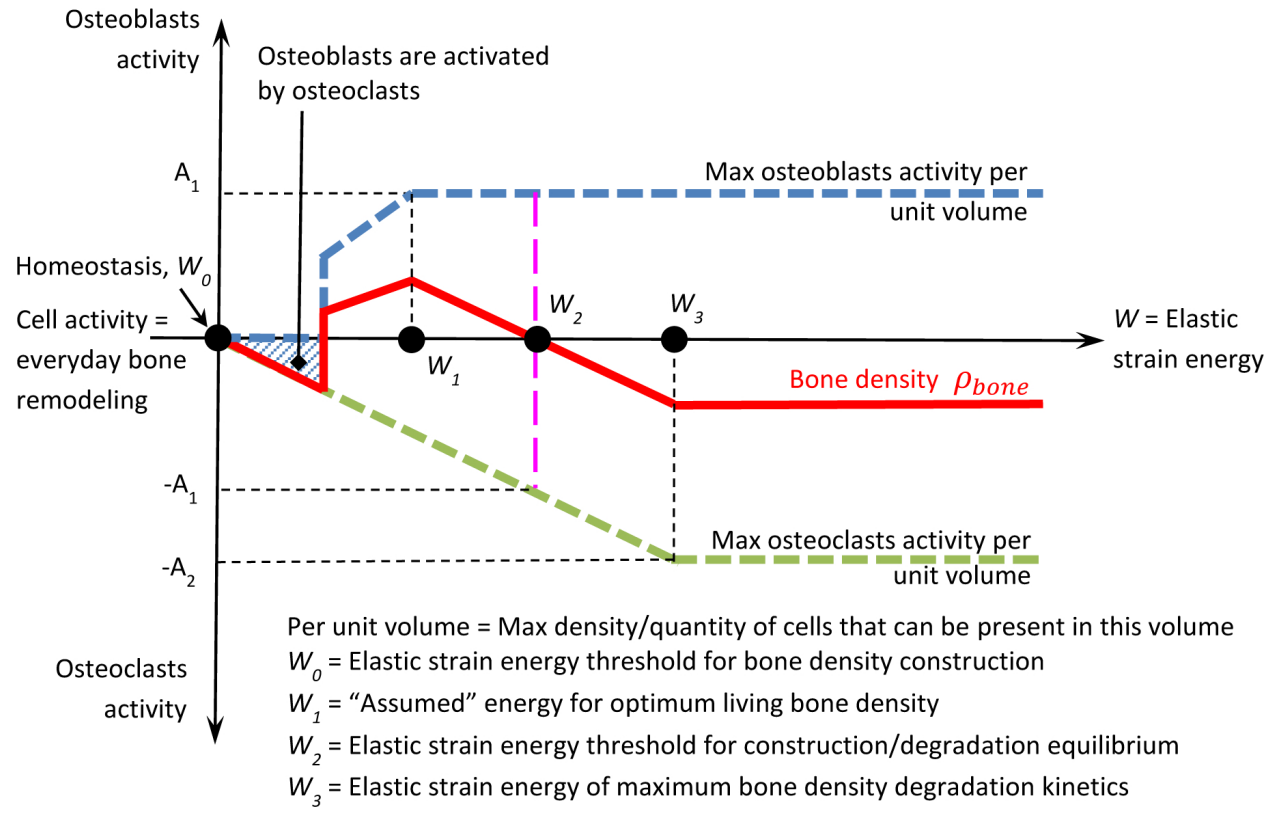

Figure 1. Schematic of the mechanoregulatory model of cellular activity as a function of the developed mechanical energy $W$ within the structure. Blue dashed line: osteoblast activity $A_{\mathrm{ob}}$. Green dashed line: osteoclast activity $A_{\mathrm{oc}}$. Red line: bone density $\rho_{\text {bone }}$ obtained by the combined blue and green cellular activities.

up to the maximum value (defined by the porosity). Finally, as we are working at the continuous macroscopic scale, we define a continuous representative volume element (RVE) that is large enough so that both osteoblasts and osteoclasts can be active within it at the same time (as a function of the given bone microstructure distribution and the given mechanical strain field) depending on the signals transmitted by the osteocytes. It is therefore assumed that the total bone mineral density change is the sum of the positive (through osteoblasts) and the negative (through osteoclasts) effects within this given RVE. Therefore, bone mineral density is directly proportional to the cell activity and defined by the corresponding units (i.e., the cellular activity is given in $\mathrm{kg} \cdot \mathrm{m}^{-3} \cdot$ unit time $^{-1}$ of fabricated (or degraded) bone per cell density).

A schematic of the cell activities as a function of the intensity of the developed mechanical energy $W$ within the structure is presented in Figure 1. The homeostasis condition $\left(W_{0}\right)$ corresponds to the equilibrium state where it is acknowledged that bone remodeling and cellular activity are nonzero, but they are not integrated within the model. The modeled variations (i.e., bone density change) are dependent on cell activity differences away from the equilibrium state. 
The osteoblast activity is shown as positive whereas the osteoclast activity is shown as negative. Both activities increase linearly up to a maximum level as the mechanical energy increases. Two conditions are required here:

(1) for a bone density increase for "medium low" mechanical energy ( $W_{0}<W<$ $W_{2}$ ), the osteoblast activity must be higher than the osteoclast one, and

(2) for a bone density decrease with "higher" mechanical energy $\left(W>W_{2}\right)$, the osteoclast activity must be higher than the osteoblast one.

On the graph in Figure 1, the initial osteoblast activity is triggered by osteoclasts (osteoblasts will start being active only after osteoclasts have "cleaned up" the bone surface so bone remodeling can initiate). At $W_{0}$, we are at homeostasis state where neither bone formation nor resorption occurs at a constant living load condition. Only continuous bone remodeling of living is present. This means that Figure 1 is correct at a given time $t$ and corresponds to an equilibrium state once bone formation or degradation has finished. In the current work, this condition corresponds to the start of the analysis (initial zero condition). From $W_{0}$ to $W_{1}$, both osteoblast and osteoclast activity increase at the same time leading to an increase of the bone mineral density since the sum of both cell activities is positive. When reaching the maximum osteoblast activity, i.e., $W \geq W_{1}$, the bone mineral density increase is impacted by the osteoclast activity taking over the osteoblast one. Above a given energy threshold, from $W_{2}$ to $W_{3}$, the bone mineral density decreases as the combined cell effect is negative. For a mechanical energy between $W_{0}$ and $W_{2}$, we have a bone density increase, while for a mechanical energy above $W_{2}$, we have a bone density decrease. $W_{2}$ corresponds to the energy threshold not to overtake to keep a good bone health. The following equations interpret the scheme in Figure 1:

$\begin{array}{lllll}\text { if } W_{0}<W<W_{1}, & \text { then } & A_{\mathrm{ob}}=k_{1} \cdot W+\rho_{\mathrm{bone}}^{\mathrm{ini}} & \text { and } & A_{\mathrm{oc}}=-k_{2} \cdot W+\rho_{\mathrm{bone}}^{\mathrm{ini}}, \\ \text { if } W_{1}<W<W_{2}, & \text { then } & A_{\mathrm{ob}}=A_{1}+\rho_{\mathrm{bone}}^{\mathrm{ini}} & \text { and } & A_{\mathrm{oc}}=-k_{2} \cdot W+\rho_{\mathrm{bone}}^{\mathrm{ini}}, \\ \text { if } W_{2}<W<W_{3}, & \text { then } & A_{\mathrm{ob}}=A_{1}+\rho_{\mathrm{bone}}^{\mathrm{ini}} & \text { and } & A_{\mathrm{oc}}=-k_{2} \cdot W+\rho_{\mathrm{bone}}^{\mathrm{ini}}, \\ \text { if } W>W_{3}, & \text { then } & A_{\mathrm{ob}}=A_{1}+\rho_{\mathrm{bone}}^{\mathrm{ini}} & \text { and } & A_{\mathrm{oc}}=-A_{2}+\rho_{\mathrm{bone}}^{\mathrm{ini}}\end{array}$

where $k_{1}$ and $k_{2}$ are the coefficients of osteoblast and osteoclast activity increase, $A_{1}$ and $A_{2}$ are their corresponding maximum values, and $\rho_{\mathrm{bone}}^{\mathrm{ini}}$ is the initial bone density, constant over time, in $\mathrm{kg} \cdot \mathrm{m}^{-3} \cdot$ unit time ${ }^{-1}$. Thus, we obtain a model with only four parameters $\left(k_{1}, k_{2}, A_{1}\right.$, and $\left.A_{2}\right)$ defined as

$$
\begin{aligned}
& A_{\mathrm{ob}}=k_{1} \cdot W+\rho_{\mathrm{bone}}^{\mathrm{ini}} \quad \text { for } W<W_{1}, \quad A_{\mathrm{oc}}=-k_{2} \cdot W+\rho_{\mathrm{bone}}^{\mathrm{ini}} \quad \text { for } W<W_{3}, \\
& A_{\mathrm{ob}}^{\mathrm{max}}=A_{1}+\rho_{\mathrm{bone}}^{\mathrm{ini}} \quad \text { for } W>W_{1}, \quad A_{\mathrm{oc}}^{\mathrm{max}}=-A_{2}+\rho_{\mathrm{bone}}^{\mathrm{ini}} \quad \text { for } W>W_{3} .
\end{aligned}
$$

The units of these four parameters are given in $\mathrm{kg} \cdot \mathrm{m}^{-3} \cdot$ unit time ${ }^{-1}$ for $A_{1}$ and $A_{2}$, and in $\mathrm{kg} \cdot \mathrm{mJ}^{-1} \cdot \mathrm{m}^{-3} \cdot$ unit time ${ }^{-1}$ for $k_{1}$ and $k_{2}$. Such parameters can be quantified 


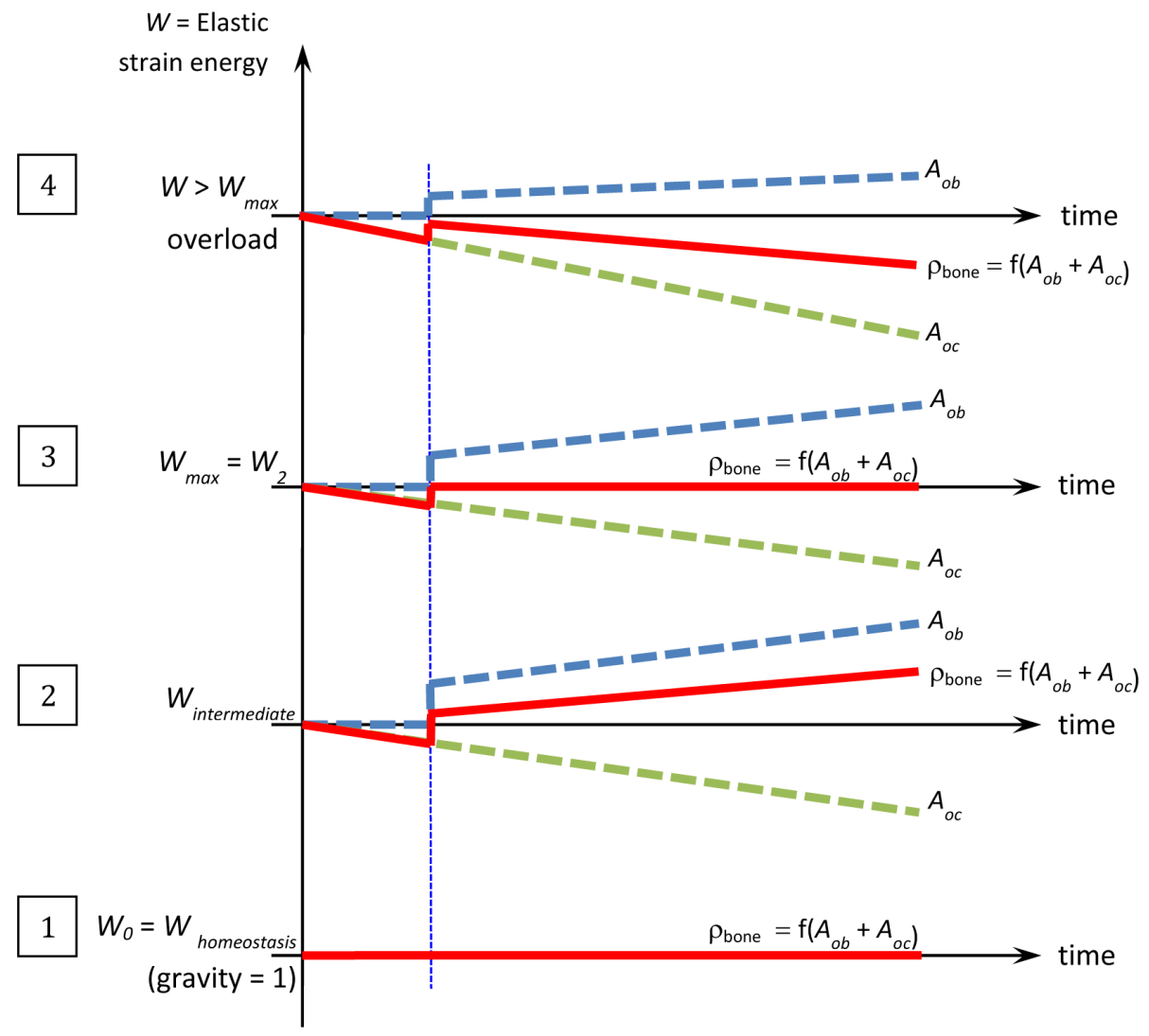

Figure 2. Schematic of the mechanoregulatory model or cellular activity as a function of time $t$ for different levels of applied mechanical load (energy) within the structure. Blue dashed line: osteoblast activity $A_{\mathrm{ob}}$. Green dashed line: osteoclasts activity $A_{\mathrm{oc}}$. Red line: bone density $\rho_{\text {bone }}$.

experimentally by histochemistry through the measurement of the different cell density activities as a function of the applied mechanical load at any given time and point of the structure. For instance, osteocyte viability and osteoclast activity can be determined through the ratio between empty (i.e., without cell) and full (i.e., with cell) lacunae. More specifically, osteocyte viability can be determined by cell apoptosis through cleaved caspase-3 activation [Lavrik 2005; Nicholson et al. 1995; Maurel et al. 2013], and osteoclast activity through the measure of the resorption area surrounding the cells (TRAP activity) [Kodama et al. 2009].

Once the cell activity has been defined and quantified as a function of the mechanical energy $W$, we can describe it as a function of time for different levels of mechanical loads (as presented in Figure 2). 
Four different cases are considered:

(1) When forces correspond to everyday life conditions, we are at equilibrium state ( $\left.W_{\text {homeostasis }}\right)$ where the bone density is in the homeostasis condition. In this case, the "average" osteoblastic and osteoclastic cell activities are equal and opposite. Their sum is equal to zero (corresponding to homeostatic state) and bone mineral density remains constant over time.

(2) When forces increase ( $\left.W_{\text {intermediate }}\right)$, corresponding to a "sport training"-like activity at a moderate level, osteoblastic cells become predominant and the sum of these effects is positive leading to an increase in the bone mineral density.

(3) If the "exercise" level increases (over-training-like) leading to an increase of the averaged developed mechanical energy, the energy level reaches a threshold $\left(W_{\max }=W_{2}\right)$ corresponding to the maximum energy under which bone density evolution remains at best positive or null. Here again, and similarly to the homeostasis case, but not for the same reason, the combined effect of osteoblasts and osteoclasts provides equilibrium. However, contrary to the homeostasis case, this equilibrium is unstable, depending on the biology that is patient-dependent.

(4) Finally, when the average mechanical energy goes beyond the threshold ( $W=$ $W_{2}$ ), bone density decreases due to an over-expression of the osteoclastic cells reacting to a solicitation that needs to be biologically quantified.

In essence, an average person's life is the result of the combined effect of all these different cases (being dependent on both the mechanical energy level and time) and bone mineral density evolves accordingly in space and time.

An interpretation of the schematic bone mineral density evolution in time of Figure 2 is given by the four energy intervals

$$
\begin{aligned}
& \text { if } W_{0}<W<W_{1} \text {, then } \frac{\partial \rho_{\text {bone }}}{\partial t}>0 \text { and } \rho_{\text {bone }}>0 \text {, } \\
& \text { if } W_{1}<W<W_{2} \text {, then } \frac{\partial \rho_{\text {bone }}}{\partial t}<0 \text { and } \rho_{\text {bone }}>0 \text {, } \\
& \text { if } W_{2}<W<W_{3} \text {, then } \frac{\partial \rho_{\text {bone }}}{\partial t}<0 \text { and } \rho_{\text {bone }}<0 \text {, } \\
& \text { if } W>W_{3}, \quad \text { then } \frac{\partial \rho_{\text {bone }}}{\partial t}<0 \text { and } \rho_{\text {bone }}<0 \text {, }
\end{aligned}
$$

where $\rho_{\text {bone }}$ is the bone density.

When combining the two schemes of Figures 1 and 2 on the same graph, we obtain the corresponding Figure 3 where the bone mineral density is a function 


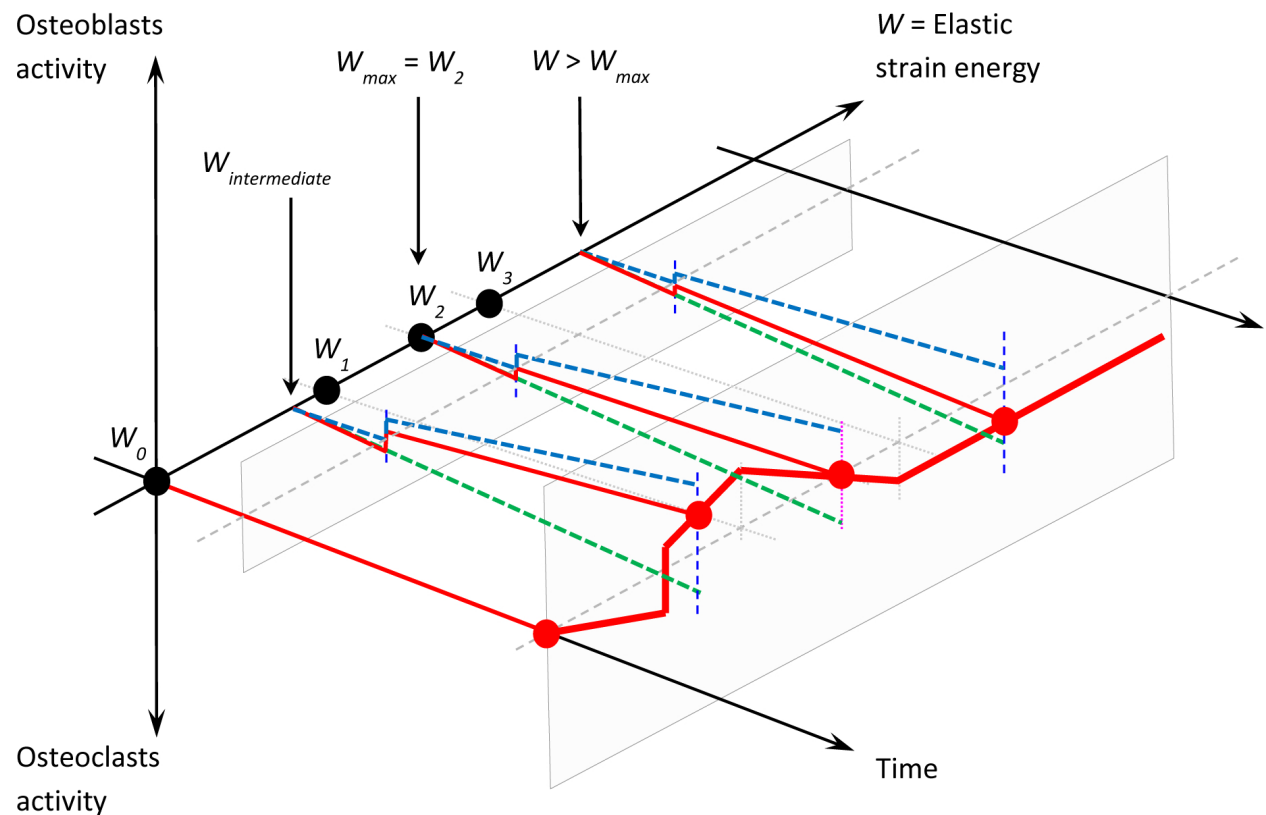

Figure 3. Global schematic of the mechanoregulatory model or cell activity as a function of time and energy. Blue dashed line: osteoblast activity $A_{\mathrm{ob}}$. Green dashed line: osteoclast activity $A_{\mathrm{oc}}$. Red line: bone density $\rho_{\text {bone. }}$

of time $t$ and the elastic strain energy $W$ (when we suppose that the mechanical behavior of bone is linear elastic).

As the cell activity is defined as an amount of fabricated (or degraded) bone as a function of its density, the amount of mechanical energy developed, and the time, the variation of bone mineral density can be computed directly. It remains only to calculate the corresponding Young's modulus (as a function of the bone density), given by the relation $E=E_{0} \cdot \rho_{\text {bone }}^{2}$ [Rho et al. 1995], that is classically accepted in the literature, where $E_{0}$ is the cortical bone Young's modulus.

2.2. Model application. A numerical application of the above proposed model is made on a simplified geometry accounting for simple load conditions. We consider a femur diaphysis loaded under compression (i.e., body weight) as presented in Figure 4, left. Since we assume a constant distribution of the stresses through the thickness of the femur diaphysis, we propose to study a simplified 2D rectangular beam of length $\mathrm{L}=50 \mathrm{~mm}$ and height $\mathrm{H}=20 \mathrm{~mm}$ (Figure 4, right).

Of course, it is acknowledged that the exact quantification of the model parameters will depend on the given geometry and structure of the exact experimental model to represent. In this case, we will quantify them only for validation purposes. 


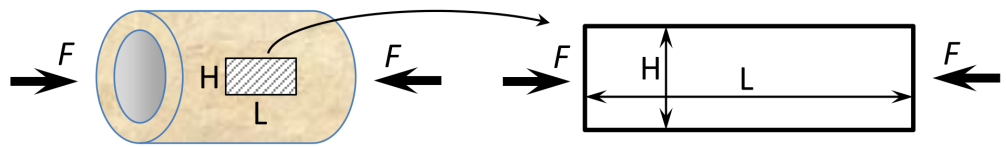

Figure 4. Geometry for the numerical application. Left: real geometry of a femoral diaphysis under simple tension. Right: simplified geometry of a rectangular beam under simple tension.

The other material parameters are defined by the Young's modulus of the cortical bone $\mathrm{E}_{0}=20.3 \mathrm{GPa}$ and Poisson ratio $v=0.3$ [Bernard et al. 2013]. The applied mechanical load is equivalent to a human body weight $F=400 \mathrm{~N}$ on one leg and an initial normalized bone density $\rho_{\text {bone }}^{\text {ini }}=0.5$ is taken for validation purposes. We suppose a closed system with no external input.

The model parameters were identified without a priori knowledge of the corresponding biological quantifications of the in vivo conditions and serve here only for validation. The homeostatic energy $W_{0}=1 \times 10^{-5} \mathrm{~mJ}$ was determined based on an average femur cross-sectional area and its corresponding bone density and body weight load conditions. The other two energies $W_{1}=1.456 \times 10^{-5} \mathrm{~mJ}$ and $W_{3}=3 \times 10^{-5} \mathrm{~mJ}$ were defined based on identification of the four parameters of the model by $k_{1}=1 \times 10^{5} \mathrm{~kg} \cdot \mathrm{mJ}^{-1} \cdot \mathrm{m}^{-3} \cdot$ unit time ${ }^{-1}, k_{2}=0.7 \times 10^{5} \mathrm{~kg} \cdot \mathrm{mJ}^{-1} \cdot \mathrm{m}^{-3}$. unit time ${ }^{-1}, A_{1}=1.456 \mathrm{~kg} \cdot \mathrm{m}^{-3} \cdot$ unit time $^{-1}$, and $A_{2}=2 \mathrm{~kg} \cdot \mathrm{mJ}^{-1} \cdot \mathrm{m}^{-3} \cdot$ unit time $^{-1}$, where $k_{1}>k_{2}$ and $A_{2}>A_{1}$. The intermediate energy $W_{2}$ is the linear interpolation between $W_{1}$ and $W_{3}$. The model was implemented within the software COMSOL Multiphysics, and the results are presented in the following section.

\section{Results and discussion}

3.1. Validation. The model was subjected to a constant force $F=400 \mathrm{~N}$, corresponding to the body weight, for an arbitrary length of time, leading to a constant energy distribution throughout the structure and hence a bone density evolution as defined by Figure 3. An initial numerical validation of the model was made by testing three simplified cases: (i) osteoblasts and osteoclasts have the same activity (i.e., the models parameters are equal and opposite: $k_{1}=-k_{2}$ and $A_{1}=-A_{2}$ ), (ii) only osteoblasts are active (i.e., $k_{2}=A_{2}=0$ ), and (iii) only osteoclasts are active (i.e., $k_{1}=A_{1}=0$ ). The results are presented in Figure 5.

As expected, an equal intensity of osteoblast and osteoclast activity (Figure 5, left) leads to a constant bone density as a function of time. This is similar to homeostasis conditions when homogenized to long periods of time (everyday life activity). In Figure 5, center, when only osteoblasts are considered (i.e., $k_{2}=A_{2}=0$ ), there is no osteoclast activity; therefore, if the energy level is high enough to trigger 


\begin{tabular}{|c|c|c|c|c|c|}
\hline & \multirow{3}{*}{$\rho_{\text {bone }}(\% / 100)$} & 0.6 & & 0.67 & 0.6 \\
\hline & & 0.5 & & 0.5 & 0.5 \\
\hline & & $\begin{array}{l}0.4 \\
0.3\end{array}$ & & $\begin{array}{l}0.4 \\
0.3\end{array}$ & 0.4 \\
\hline & $\mathrm{W}^{*} 2 \mathrm{E}-5(\mathrm{~mJ})$ & 0.2 & & $0.2 \cdots$ & 0.2 \\
\hline - & $A_{0}\left(k g \cdot m^{-3} \cdot\right.$ unit-time $\left.{ }^{-1}\right)$ & $\begin{array}{r}0.1 \\
0\end{array}$ & Artificial time & Artificial time & Artificial time \\
\hline & $A_{o c}\left(\mathrm{~kg} \cdot \mathrm{m}^{-3} \cdot\right.$ unit-time $\left.^{-1}\right)$ & & 0 & $\begin{array}{llll}-0.1 & 0 & 1 & 2 \\
-0.2 & & -3\end{array}$ & $\begin{array}{llll}-0.1 & 0 & 1 & 2 \\
-0.2 & \end{array}$ \\
\hline & & & $k_{1}=-k_{2}$ and $A_{1}=-A_{2}$ & $k_{2}=A_{2}=0$ & $k_{1}=A_{1}=0$ \\
\hline
\end{tabular}

Figure 5. Evolution of the model parameters, bone density, and mechanical energy as a function of time for the three simplified cases.

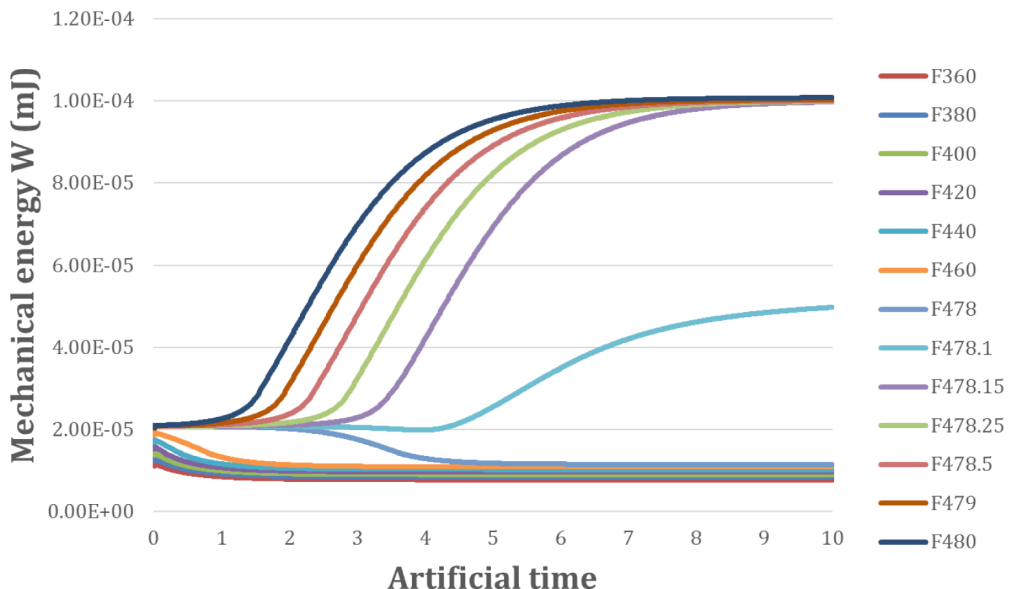

Figure 6. Mechanical energy $W$ variation as function of time for different values of the applied force $F$.

osteoblasts, bone density increases and since the bone stiffness increases correspondingly, then the developed mechanical energy (for a constant applied force) decreases, and so does the osteoblast activity. Finally, when only osteoclasts are considered (i.e., $k_{1}=A_{1}=0$, in Figure 5, right), there is no osteoblast activity, bone density decreases and so does its stiffness, and hence the developed mechanical energy increases (for a constant applied force) together with the osteoclast activity.

3.2. Results and sensitivity study for different load cases. Different intensities of $F$ were applied from $360 \mathrm{~N}$ to $480 \mathrm{~N}$. The results are presented in Figures 6, 7, and 8 . For constant-load cases below $478 \mathrm{~N}$, we observe a convergence towards an increase of bone density (Figure 7) and a corresponding decrease of mechanical energy (Figure 6). For load cases above 478 N, the opposite occurs with a decrease 


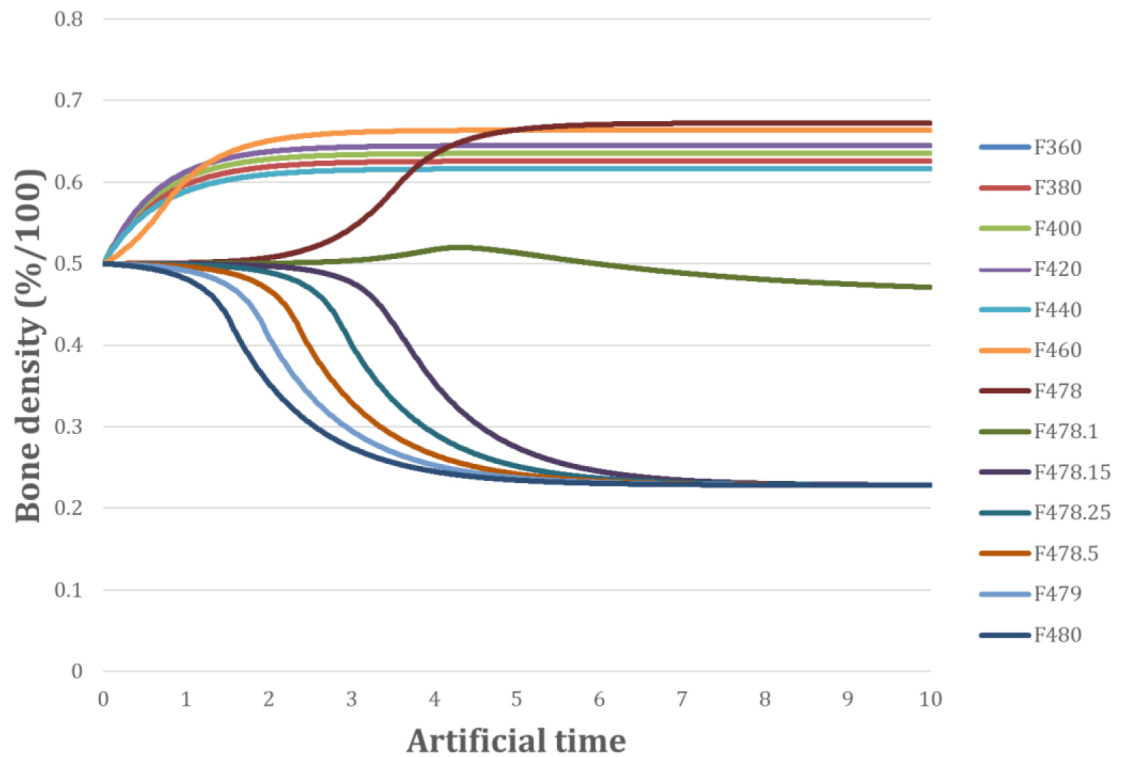

Figure 7. Bone mineral density $\rho_{\text {bone }}$ variation as function of time for different values of the applied force $F$.

of bone density and an increase of energy. Here, the energy threshold $W_{2}$ shows up around $478.1 \mathrm{~N}$ with the given values of the model parameters.

The threshold (i.e., the switch from positive to negative bone density) is very sensitive to the intensity of the applied force and it is located within a very thin force range around $478 \mathrm{~N}$, deregulating the system very quickly. It is not possible at this stage to know if this is a limitation of the proposed model (due to the simplified assumptions made or the lack of more detailed information on the existing couplings at the mechanobiological level) or if this is really occurring in reality. Such an aspect needs to be investigated further experimentally at a later stage. For the case of the control force $(400 \mathrm{~N})$, the mechanical energy $W$ and the bone density $\rho_{\text {bone }}$ reach final values of $9.019 \times 10^{-6} \mathrm{~mJ}$ and 0.64 .

Cell activities are presented in Figure 8. For the control case, their final values are equal to $9.019 \times 10^{-1} \mathrm{~kg} \cdot \mathrm{m}^{-3} \cdot$ unit time ${ }^{-1}$ and $-6.313 \times 10^{-1} \mathrm{~kg} \cdot \mathrm{m}^{-3}$. unit time ${ }^{-1}$ for $A_{\mathrm{ob}}$ and $A_{\mathrm{oc}}$, respectively. Thus, we can conclude that for this specific case, a fast stabilization is observed to reach the mechanobiological equilibrium. Since we observed a fast switch in the variable trends around $478 \mathrm{~N}$, we decided to perform more simulations around this threshold in order to highlight such a transition.

Similarly to the developed mechanical energy and bone mineral density variations, when the applied mechanical force increases above $478 \mathrm{~N}$, osteoblast activity reaches its maximum value, and we observe an inversion of the osteoclast activity 


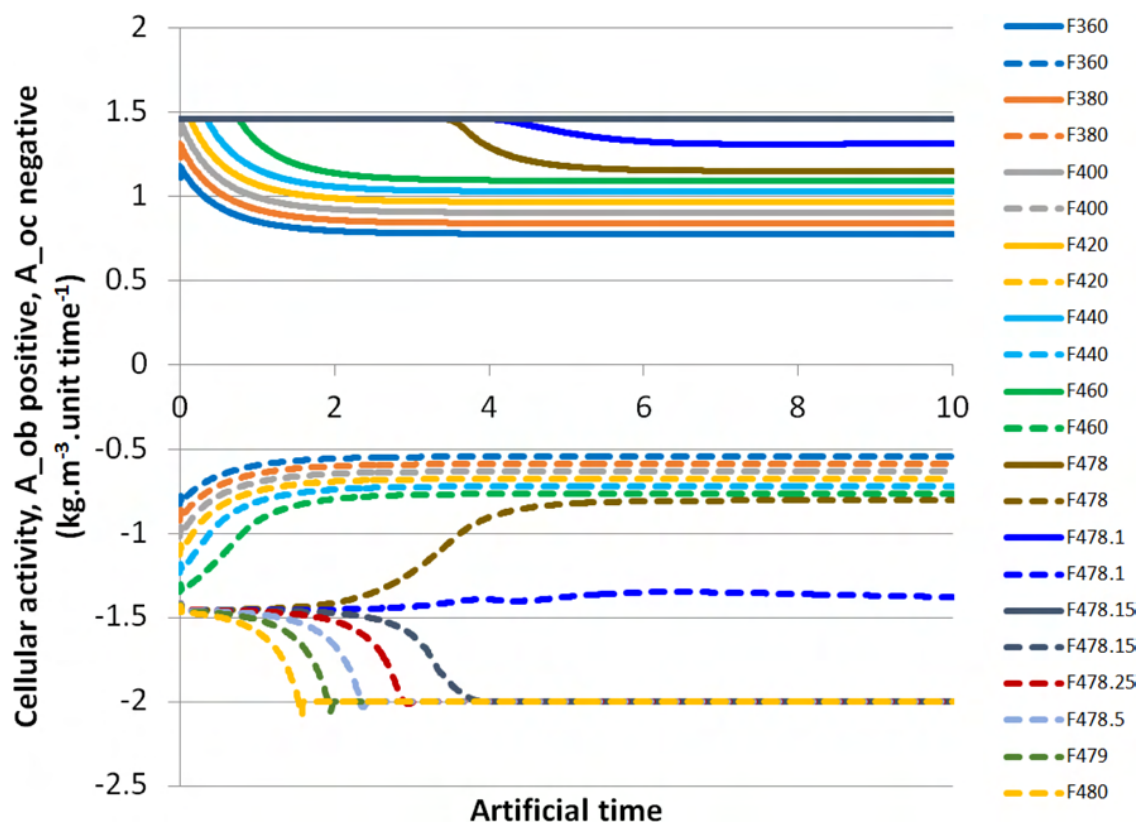

Figure 8. Osteoblast activity $A_{\mathrm{ob}}$ and osteoclast activity $A_{\mathrm{oc}}$ variations as functions of time for different values of the applied force $F$. Osteoblast activity is shown positive in continuous lines up to a force of $478.15 \mathrm{~N}$ above which it remains maximal at $1.46 \mathrm{~kg} \cdot \mathrm{m}^{-3} \cdot$ unit time ${ }^{-1}$. Osteoclast activity is shown negative in dashed lines up to a force of $480 \mathrm{~N}$ showing the transient behavior through $478 \mathrm{~N}$ towards bone degradation.

(decreasing to increasing). As the osteoblast activity is at its maximum of $1.46 \mathrm{~kg}$. $\mathrm{m}^{-3} \cdot$ unit time ${ }^{-1}$, when increasing the applied force, the osteoclast activity will pass from "ineffective" (below the osteoblast activity of $-1.5 \mathrm{~kg} \cdot \mathrm{m}^{-3} \cdot$ unit-time $^{-1}$ ) to "effective" as it reaches a final value of $-2 \mathrm{~kg} \cdot \mathrm{m}^{-3} \cdot$ unit time $^{-1}$. Once the new equilibrium reached, where both osteoblast and osteoclast activities have reached their maximum value, a fast bone degradation is observed (Figure 7). A return to normal physical conditions depends upon a decrease of the applied mechanical force and a new stabilization of the system under study.

The proposed model only relies on a few parameters: $\rho_{\text {bone }}^{\text {ini }}, A_{1}, A_{2}, k_{1}, k_{2}$, $W_{0}, W_{1}$, and $W_{3}$. A sensitivity study was performed in order to evaluate their influence on the overall results. We vary one parameter at a time in the range $\pm 10 \%$ and we recorded the obtained results for the energy $W$, the osteoblast $\left(A_{\mathrm{ob}}\right)$ and osteoclast $\left(A_{\mathrm{oc}}\right)$ activities, and the bone density $\rho_{\text {bone }}$. The obtained results show that $\rho_{\text {bone }}^{\text {ini }}, A_{1}, A_{2}, W_{0}, W_{1}$, and $W_{3}$, within the $10 \%$ range variation, have 


\begin{tabular}{|c|rcccc|}
\hline$k_{1}$ & $\begin{array}{r}9 \times 10^{4} \\
(-10 \%)\end{array}$ & $\begin{array}{c}9.5 \times 10^{4} \\
(-5 \%)\end{array}$ & $\begin{array}{c}10 \times 10^{4} \\
(\text { control })\end{array}$ & $\begin{array}{c}10.5 \times 10^{4} \\
(+5 \%)\end{array}$ & $\begin{array}{c}11 \times 10^{4} \\
(+10 \%)\end{array}$ \\
\hline$W\left(\mathrm{~kg} \cdot \mathrm{mJ}^{-1} \cdot \mathrm{m}^{-3} \cdot \mathrm{ut}^{-1}\right)$ & $+11.76 \%$ & $+5.39 \%$ & $9.019 \times 10^{-6}$ & $-4.63 \%$ & $-8.67 \%$ \\
$\rho_{\text {bone }}(\times 100 \%)$ & $-5.42 \%$ & $-2.58 \%$ & 0.6352 & $+2.41 \%$ & $+4.64 \%$ \\
$A_{\mathrm{ob}}\left(\mathrm{kg} \cdot \mathrm{m}^{-3} \cdot \mathrm{ut}^{-1}\right)$ & $+0.61 \%$ & $+0.12 \%$ & 0.9019 & $+0.13 \%$ & $+0.47 \%$ \\
$A_{\mathrm{oc}}\left(\mathrm{kg} \cdot \mathrm{m}^{-3} \cdot \mathrm{ut}^{-1}\right)$ & $-11.80 \%$ & $-5.39 \%$ & -0.6313 & $+4.63 \%$ & $+8.66 \%$ \\
\hline
\end{tabular}

\begin{tabular}{|c|rcccr|}
\hline$k_{2}$ & $\begin{array}{r}6.3 \times 10^{4} \\
(-10 \%)\end{array}$ & $\begin{array}{c}6.65 \times 10^{4} \\
(-5 \%)\end{array}$ & $\begin{array}{c}7 \times 10^{4} \\
(\text { control })\end{array}$ & $\begin{array}{c}7.35 \times 10^{4} \\
(+5 \%)\end{array}$ & $\begin{array}{r}7.7 \times 10^{4} \\
(+10 \%)\end{array}$ \\
\hline$W\left(\mathrm{~kg} \cdot \mathrm{mJ}^{-1} \cdot \mathrm{m}^{-3} \cdot \mathrm{ut}^{-1}\right)$ & $-6.31 \%$ & $-3.32 \%$ & $9.019 \times 10^{-6}$ & $+3.68 \%$ & $+7.82 \%$ \\
$\rho_{\mathrm{bone}}(\times 100 \%)$ & $+3.32 \%$ & $+1.7 \%$ & 0.6352 & $-1.78 \%$ & $-3.68 \%$ \\
$A_{\mathrm{ob}}\left(\mathrm{kg} \cdot \mathrm{m}^{-3} \cdot \mathrm{ut}^{-1}\right)$ & $-6.31 \%$ & $-3.32 \%$ & 0.9019 & $+3.68 \%$ & $+7.82 \%$ \\
$A_{\mathrm{oc}}\left(\mathrm{kg} \cdot \mathrm{m}^{-3} \cdot \mathrm{ut}^{-1}\right)$ & $+15.68 \%$ & $+8.14 \%$ & -0.6313 & $-8.87 \%$ & $-18.60 \%$ \\
\hline
\end{tabular}

Table 1. Results of the sensitivity study performed for $k_{1}$ and $k_{2}$, where "ut" stands for "unit time".

little effect. The initial bone mineral density value has no effect since we apply a constant force on the structure, so whatever the initial state equilibrium, it will lead to the same final results with a different kinematic evolution. Since most of the evolution occurs during the osteoblast $\left(A_{\mathrm{ob}}\right)$ and osteoclast $\left(A_{\mathrm{oc}}\right)$ activities through $k_{1}$ and $k_{2}$ parameters, the other parameters do not impact either of the obtained results in this study.

However, it appeared that $k_{1}$ and $k_{2}$ have a higher impact on the final results as reported in Table 1. As $k_{1}$ decreases, $\rho_{\text {bone }}$ decreases too $(-11.8 \%)$, whereas as $k_{2}$ decreases, $\rho_{\text {bone }}$ increases $(+15.68 \%)$. For $W$ and $A_{\mathrm{ob}}$, inverse trends can be noticed when varying $k_{1}$ and $k_{2}$. For $k_{1}, W$ and $A_{\mathrm{ob}}$ fluctuate between $+11.76 \%$ and $-8.67 \%$ and between $-5.42 \%$ and $+4.64 \%$, respectively. For $k_{2}, W$ and $A_{\mathrm{ob}}$ fluctuate between $-6.31 \%$ and $+7.82 \%$ and between $+3.32 \%$ and $-3.68 \%$, respectively. Finally, $A_{\mathrm{oc}}$ varies between $+0.61 \%$ and $+0.47 \%$ for $k_{1}$ and between $+6.31 \%$ and $+7.82 \%$ for $k_{2}$.

Most of the bone mineral density variation occurs within the $k_{1}$ and $k_{2}$ phases. Hence, modifying only one parameter $\left(k_{1}\right.$ or $\left.k_{2}\right)$ at a time has a direct impact on the final results. This effect would be compensated for if both parameters would change oppositely in the same proportions, which would lead to the same final results.

After identification and validation of the mechanobiological phenomena at play in this model (developed mechanical energy, osteoblast and osteoclast activities, and bone mineral density variations) as a function of time for given intensities of mechanical forces, the results were plotted not as a function of time, but as a 


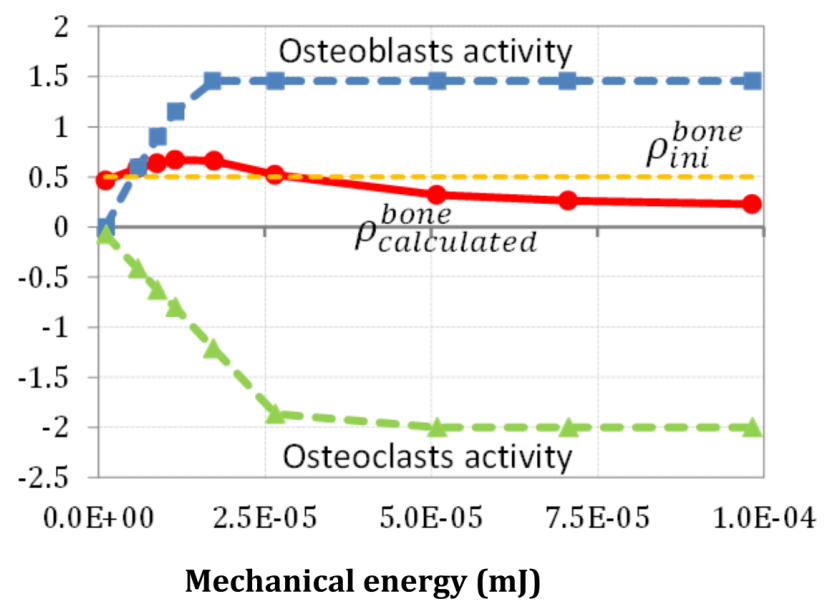

Figure 9. Numerical results (in comparison to the initial model hypotheses of Figure 1).

function of the intensity of mechanical energy for all the simulations. The results are presented in Figure 9.

Figure 9 shows the results obtained with the newly proposed mechanoregulatory model. These results should be compared with Figure 1 showing the theoretical hypotheses defined initially. An observation is made that a very strong correlation is obtained between the proposed model and the obtained numerical results. We have an initial increase of the osteoblast and osteoclast activities up to their maximum values that remain constant afterwards. The only difference is that in the numerical model, the initial osteoblast activity being triggered by the osteoclasts was not included, which explains why it is not visible in Figure 9, but starts directly increasing at the origin. We also observe that the bone mineral density evolution follows the sum of both cell activities (function of the applied mechanical force) being positive initially for lower mechanical energy and negative later on for higher energy from an original normalized bone density of 0.5 . The maximum osteoblast activity is reached first (energy level $W_{1}$ ) followed by the maximum of the osteoclast activity (energy level $W_{3}$ ). We note that the equilibrium between osteoblast and osteoclast activity does not correspond to the bone mineral density variation equaling zero (i.e., a return to the initial bone density of 0.5 ) as there appears to be a time delay of the structure response to the mechanical load. The passage of the bone mineral density from positive to negative variation occurs later. Thereafter, there is a continuing decrease of the bone mineral density with stabilization later on, dependent on the intensity of the applied force.

3.3. Application to variable load conditions. The numerical results were validated on constant load and showed good correlation with the proposed model hypotheses. 


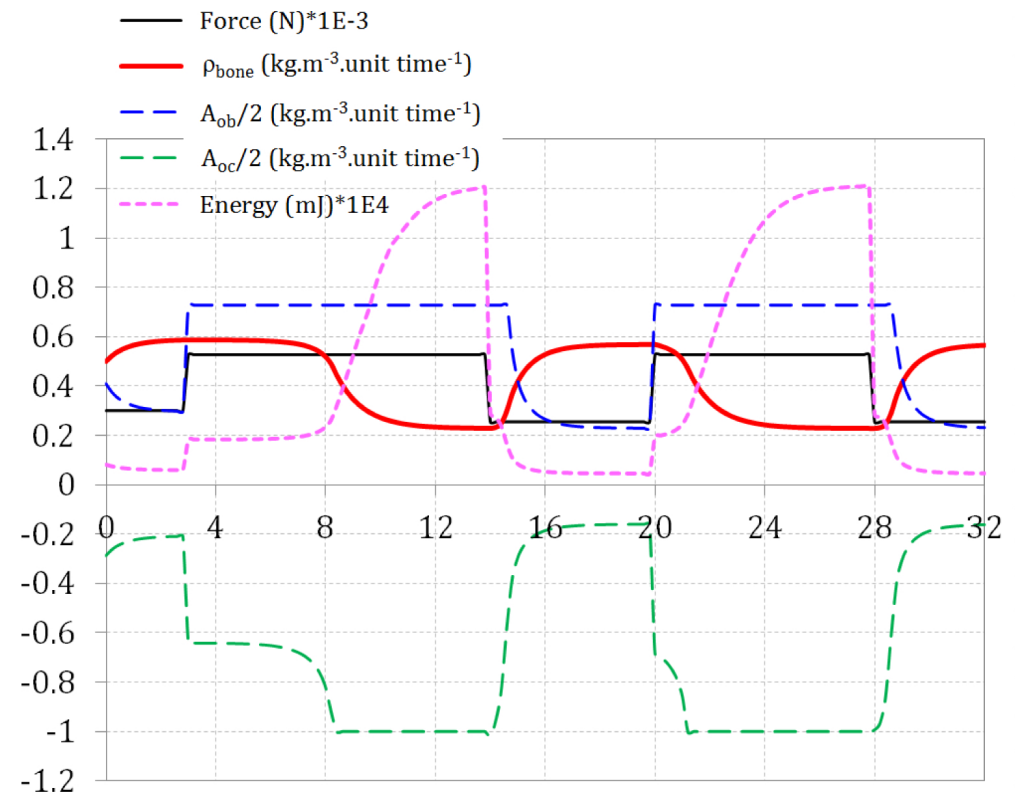

Artificial time

Figure 10. Bone mineral density, cell activity, and energy variations when applying a variable force below and above the degradation threshold (overload threshold).

Hence, to extract bone density variations in a more "real application"-like environment, variable mechanical forces were considered. A constant force is applied on the model until equilibrium is reached (constant bone density that occurs in normal living conditions after several weeks). Then the intensity of the applied force is changed to reach a new equilibrium. This is done for two intensities of the mechanical force, one below and one above the overload degradation threshold. Results are presented in Figure 10.

At initial mechanical load $(300 \mathrm{~N})$, bone density quickly reaches equilibrium $(\approx 0.55)$ with decreasing cellular activity. Next, the force is increased above the threshold $(520 \mathrm{~N})$. The cell activity is changed to reach its maximum with immediate response from osteoblasts and delayed response from osteoclasts. This is dependent on the evolution of the elastic strain energy within the model that takes time to develop as a function of the bone density that is present at a given point of the structure and at a given time. Once the energy has developed above the threshold, bone density starts to decrease to reach a new equilibrium that is lower than the previous one. When cycling this effect by applying in turn these two mechanical forces, we observe the corresponding bone density variations and new equilibrium being formed. Decreasing the overloading force will lead to a reincrease of the 
bone density coming back to its "normal" healthy working conditions. However, it is anticipated that keeping an overload condition on the bone will probably lead to a complete degradation of the structure. This was not modeled here as it certainly requires accounting for extra mechanobiological actions that were not integrated within this simplified mechanoregulatory model.

\section{Conclusion}

A new comprehensive approach based on cell activity to describe bone remodeling is proposed to assess the possible bone degradation kinetics when under high-intensity mechanical loads. Despite the complexity of the mechanobiological process, only four experimentally measurable parameters are required to tune this model for specific cases. These are the variations of the bone density kinetics with the intensity of the applied mechanical loads up to their maximum value, and the two maximum values for osteoblasts and osteoclasts activities. The results show the respective contributions of each process on the bone mineral density evolution and are in agreement with the experimental data provided in the literature. In fact, the model is able to depict both the harmful and the favorable effects of high and medium mechanical loads, respectively. With this approach, when experimentally measuring the four model parameters for different load scenario (force intensity, test specimen, aging, diseased, etc.), potential differences are expected between the cases and possible foreseen applications for the optimization process for sport activities.

\section{Acknowledgements}

The authors would like to thank the CNRS for its financial support through the Défi Mécanobiologie to carry out the work.

\section{References}

[Allena and Maini 2014] R. Allena and P. K. Maini, "Reaction-diffusion finite element model of lateral line primordium migration to explore cell leadership", B. Math. Bio. 76:12 (2014), 30283050 .

[Andreaus et al. 2014a] U. Andreaus, M. Colloca, and D. Iacoviello, "Optimal bone density distributions: numerical analysis of the osteocyte spatial influence in bone remodeling", Comput. Meth. Prog. Bio. 113:1 (2014), 80-91.

[Andreaus et al. 2014b] U. Andreaus, I. Giorgio, and T. Lekszycki, "A 2-D continuum model of a mixture of bone tissue and bio-resorbable material for simulating mass density redistribution under load slowly variable in time”, Z. Angew. Math. Mech. 94:12 (2014), 978-1000.

[Bagherian et al. 2019] A. Bagherian, M. Baghani, D. George, Y. Rémond, C. Chappard, S. Patlazhan, and M. Baniassadi, "A novel numerical model for the prediction of patient-dependent bone density loss in microgravity based on micro-CT images", Continuum Mech. Therm. 32:3 (2019), 927-943. 
[Beaupré et al. 1990] G. S. Beaupré, T. E. Orr, and D. R. Carter, "An approach for time-dependent bone modeling and remodeling-application: a preliminary remodeling simulation", J. Orthop. Res. 8:5 (1990), 662-670.

[Bednarczyk and Lekszycki 2016] E. Bednarczyk and T. Lekszycki, "A novel mathematical model for growth of capillaries and nutrient supply with application to prediction of osteophyte onset", $Z$. Angew. Math. Phys. 67:4 (2016), art. id. 94.

[Bernard et al. 2013] S. Bernard, Q. Grimal, and P. Laugier, "Accurate measurement of cortical bone elasticity tensor with resonant ultrasound spectroscopy”, J. Mech. Behav. Biomed. 18 (2013), $12-19$.

[Burr and Allen 2014] D. B. Burr and M. R. Allen, "Bone modeling and remodeling", Chapter 4, pp. 75-90 in Basic and applied bone biology, edited by D. B. Burr and M. R. Allen, Academic, London, 2014.

[Carter 1984] D. R. Carter, "Mechanical loading histories and cortical bone remodeling", Calcified Tissue Int. 36:S1 (1984), S19-S24.

[Cowin 1986] S. C. Cowin, "Wolff's law of trabecular architecture at remodeling equilibrium", $J$. Biomech. Eng. 108:1 (1986), 83-88.

[Ehrlich and Lanyon 2002] P. J. Ehrlich and L. E. Lanyon, "Mechanical strain and bone cell function: a review", Osteoporosis Int. 13:9 (2002), 688-700.

[Forwood and Parker 1989] M. R. Forwood and A. W. Parker, "Microdamage in response to repetitive torsional loading in the rat tibia", Calcified Tissue Int. 45:1 (1989), 47-53.

[Frame et al. 2018] J. Frame, P.-Y. Rohan, L. Corté, and R. Allena, "Optimal bone structure is dependent on the interplay between mechanics and cellular activities", Mech. Res. Commun. 92 (2018), 43-48.

[Frame et al. 2019] J. Frame, P.-Y. Rohan, L. Corté, and R. Allena, "A mechano-biological model of multi-tissue evolution in bone", Continuum Mech. Therm. 31:1 (2019), 1-31.

[Frost 1987] H. M. Frost, "Bone 'mass' and the 'mechanostat': a proposal”, Anat. Rec. 219:1 (1987), $1-9$.

[George et al. 2017] D. George, C. Spingarn, C. Dissaux, M. Nierenberger, R. A. Rahman, and Y. Rémond, "Examples of multiscale and multiphysics numerical modeling of biological tissues", Bio-Med. Mater. Eng. 28:S1 (2017), S15-S27.

[George et al. 2018a] D. George, R. Allena, and Y. Rémond, "Cell nutriments and motility for mechanobiological bone remodeling in the context of orthodontic periodontal ligament deformation”, J. Cell. Immunoth. 4:1 (2018), 26-29.

[George et al. 2018b] D. George, R. Allena, and Y. Rémond, "A multiphysics stimulus for continuum mechanics bone remodeling”, Math. Mech. Complex Syst. 6:4 (2018), 307-319.

[George et al. 2019] D. George, R. Allena, and Y. Rémond, "Integrating molecular and cellular kinetics into a coupled continuum mechanobiological stimulus for bone reconstruction", Continuum Mech. Therm. 31:3 (2019), 725-740.

[Giorgio et al. 2016] I. Giorgio, U. Andreaus, D. Scerrato, and F. dell'Isola, "A visco-poroelastic model of functional adaptation in bones reconstructed with bio-resorbable materials", Biomech. Model. Mechan. 15:5 (2016), 1325-1343.

[Giorgio et al. 2017] I. Giorgio, U. Andreaus, F. dell'Isola, and T. Lekszycki, "Viscous second gradient porous materials for bones reconstructed with bio-resorbable grafts", Extreme Mech. Let. 13 (2017), 141-147.

[Grimston et al. 1991] S. K. Grimston, J. R. Engsberg, R. Kloiber, and D. A. Hanley, "Bone mass, external loads, and stress fracture in female runners", Int. J. Sport Biomech. 7:3 (1991), 293-302. 
[Hao et al. 2017] L. Hao, L. Rui-Xin, H. Biao, Z. Bin, H. Bao-Hui, L. Ying-Jie, and Z. Xi-Zheng, "Effect of athletic fatigue damage and the associated bone targeted remodeling in the rat ulna", Biomed. Eng. Online 16:1 (2017).

[Herman et al. 2010] B. C. Herman, L. Cardoso, R. J. Majeska, K. J. Jepsen, and M. B. Schaffler, "Activation of bone remodeling after fatigue: differential response to linear microcracks and diffuse damage", Bone 47:4 (2010), 766-772.

[Ignatius et al. 2005] A. Ignatius, H. Blessing, A. Liedert, C. Schmidt, C. Neidlinger-Wilke, D. Kaspar, B. Friemert, and L. Claes, "Tissue engineering of bone: effects of mechanical strain on osteoblastic cells in type I collagen matrices”, Biomaterials 26:3 (2005), 311-318.

[Klein-Nulend et al. 2013] J. Klein-Nulend, A. D. Bakker, R. G. Bacabac, A. Vatsa, and S. Weinbaum, "Mechanosensation and transduction in osteocytes", Bone 54:2 (2013), 182-190.

[Kodama et al. 2009] H. Kodama, A. Yamasaki, M. Abe, S. Niida, Y. Hakeda, and H. Kawashima, "Transient recruitment of osteoclasts and expression of their function in osteopetrotic (op/op) mice by a single injection of macrophage colony-stimulating factor", J. Bone Miner. Res. 8:1 (2009), $45-50$.

[Lavrik 2005] I. N. Lavrik, "Caspases: pharmacological manipulation of cell death", J. Clin. Invest. 115:10 (2005), 2665-2672.

[Lekszycki 2002] T. Lekszycki, "Modelling of bone adaptation based on an optimal response hypothesis", Meccanica 37:4-5 (2002), 343-354.

[Lekszycki and dell'Isola 2012] T. Lekszycki and F. dell'Isola, "A mixture model with evolving mass densities for describing synthesis and resorption phenomena in bones reconstructed with bioresorbable materials", Z. Angew. Math. Mech. 92:6 (2012), 426-444.

[Lemaire et al. 2005] T. Lemaire, S. Naïli, and A. Rémond, "Multiscale analysis of the coupled effects governing the movement of interstitial fluid in cortical bone", Biomech. Model. Mechan. 5:1 (2005), 39-52.

[Lemaire et al. 2010] T. Lemaire, S. Naili, and V. Sansalone, "Multiphysical modelling of fluid transport through osteo-articular media", An. Acad. Bras. Ciênc. 82:1 (2010), 127-144.

[Lemaire et al. 2011] T. Lemaire, E. Capiez-Lernout, J. Kaiser, S. Naili, and V. Sansalone, "What is the importance of multiphysical phenomena in bone remodelling signals expression? A multiscale perspective", J. Mech. Behav. Biomed. 4:6 (2011), 909-920.

[Lemaire et al. 2015] T. Lemaire, J. Kaiser, S. Naili, and V. Sansalone, "Three-scale multiphysics modeling of transport phenomena within cortical bone”, Math. Probl. Eng. (2015), art. id. 398970.

[Louna et al. 2016] Z. Louna, I. Goda, J.-F. Ganghoffer, and S. Benhadid, "Formulation of an effective growth response of trabecular bone based on micromechanical analyses at the trabecular level", Arch. Appl. Mech. 87:3 (2016), 457-477.

[Lu and Lekszycki 2016] Y. Lu and T. Lekszycki, "A novel coupled system of non-local integrodifferential equations modelling Young's modulus evolution, nutrients' supply and consumption during bone fracture healing”, Z. Angew. Math. Phys. 67:5 (2016), art. id. 111.

[Madeo et al. 2011] A. Madeo, T. Lekszycki, and F. dell'Isola, "A continuum model for the biomechanical interactions between living tissue and bio-resorbable graft after bone reconstructive surgery”, C. R. Mécanique 339:10 (2011), 625-640.

[Madeo et al. 2012] A. Madeo, D. George, T. Lekszycki, M. Nierenberger, and Y. Rémond, "A second gradient continuum model accounting for some effects of micro-structure on reconstructed bone remodelling”, C. R. Mécanique 340:8 (2012), 575-589. 
[Martin et al. 2017] M. Martin, T. Lemaire, G. Haïat, P. Pivonka, and V. Sansalone, "A thermodynamically consistent model of bone rotary remodeling: a 2D study", Comput. Method. Biomech. Biomed. 20:S1 (2017), S127-S128.

[Maurel et al. 2013] D. B. Maurel, D. Benaitreau, C. Jaffré, H. Toumi, H. Portier, R. Uzbekov, C. Pichon, C. L. Benhamou, E. Lespessailles, and S. Pallu, "Effect of the alcohol consumption on osteocyte cell processes: a molecular imaging study”, J. Cell. Mol. Med. 18:8 (2013), 1680-1693.

[Nicholson et al. 1995] D. W. Nicholson, A. Ali, N. A. Thornberry, J. P. Vaillancourt, C. K. Ding, M. Gallant, Y. Gareau, P. R. Griffin, M. Labelle, Y. A. Lazebnik, N. A. Munday, S. M. Raju, M. E. Smulson, T.-T. Yamin, V. L. Yu, and D. K. Miller, "Identification and inhibition of the ICE/CED-3 protease necessary for mammalian apoptosis”, Nature 376:6535 (1995), 37-43.

[Pivonka and Komarova 2010] P. Pivonka and S. V. Komarova, "Mathematical modeling in bone biology: From intracellular signaling to tissue mechanics", Bone 47:2 (2010), 181-189.

[Pivonka et al. 2008] P. Pivonka, J. Zimak, D. W. Smith, B. S. Gardiner, C. R. Dunstan, N. A. Sims, T. J. Martin, and G. R. Mundy, "Model structure and control of bone remodeling: a theoretical study", Bone 43:2 (2008), 249-263.

[Rémond et al. 2016] Y. Rémond, S. Ahzi, M. Baniassadi, and H. Garmestani, Applied RVE reconstruction and homogenization of heterogeneous materials, Wiley, Hoboken, NJ, 2016.

[Rho et al. 1995] J. Y. Rho, M. C. Hobatho, and R. B. Ashman, "Relations of mechanical properties to density and CT numbers in human bone", Med. Eng. Phys. 17:5 (1995), 347-355.

[Rochefort et al. 2010] G. Y. Rochefort, S. Pallu, and C. L. Benhamou, "Osteocyte: the unrecognized side of bone tissue", Osteoporosis Int. 21:9 (2010), 1457-1469.

[Ruimerman et al. 2005] R. Ruimerman, P. Hilbers, B. van Rietbergen, and R. Huiskes, "A theoretical framework for strain-related trabecular bone maintenance and adaptation", J. Biomech. 38:4 (2005), 931-941.

[Sansalone et al. 2015] V. Sansalone, D. Gagliardi, C. Desceliers, G. Haïat, and S. Naili, "On the uncertainty propagation in multiscale modeling of cortical bone elasticity", Comput. Method. Biomech. Biomed. 18:S1 (2015), 2054-2055.

[Scala et al. 2017] I. Scala, C. Spingarn, Y. Rémond, A. Madeo, and D. George, "Mechanicallydriven bone remodeling simulation: application to LIPUS treated rat calvarial defects", Math. Mech. Solids 22:10 (2017), 1976-1988.

[Schmitt et al. 2015] M. Schmitt, R. Allena, T. Schouman, S. Frasca, J. M. Collombet, X. Holy, and P. Rouch, "Diffusion model to describe osteogenesis within a porous titanium scaffold", Comput. Method. Biomech. Biomed. 19:2 (2015), 171-179.

[Sheidaei et al. 2019] A. Sheidaei, M. Kazempour, A. Hasanabadi, F. Nosouhi, M. Pithioux, M. Baniassadi, Y. Rémond, and D. George, "Influence of bone microstructure distribution on developed mechanical energy for bone remodeling using a statistical reconstruction method", Math. Mech. Solids 24:10 (2019), 3027-3041.

[Spingarn et al. 2017] C. Spingarn, D. Wagner, Y. Rémond, and D. George, "Multiphysics of bone remodeling: a 2D mesoscale activation simulation", Bio-Med. Mater. Eng. 28:S1 (2017), S153S158.

[Turner 1998] C. H. Turner, "Three rules for bone adaptation to mechanical stimuli", Bone 23:5 (1998), 399-407.

[Wolff 1986] J. Wolff, The law of bone remodelling, Springer, Berlin, 1986.

Received 6 Feb 2020. Revised 17 Jun 2020. Accepted 28 Aug 2020. 
DANIEL GEORGE: george@unistra.fr

ICube Laboratory, Université de Strasbourg, Centre National de la Recherche Scientifique, Strasbourg, France

RACHELE ALLENA: rachele.allena@ensam.eu Institut de Biomécanique Humaine Georges Charpak, Arts et Métiers ParisTech, Paris, France CÉlINE BOURZAC: celine.bourzac@vet-alfort.fr Institut National de la Santé et de la Recherche Médicale, Université Paris Sciences et Lettres, Centre National de la Recherche Scientifique, Paris, France

STÉPHANE PALLU: stephane.pallu@univ-orleans.fr Institut National de la Santé et de la Recherche Médicale, Université Paris Sciences et Lettres, Centre National de la Recherche Scientifique, Paris, France and

Collégium Sciences et Techniques, Université d'Orléans, Orléans, France

MORAD BENSIDHOUM: morad.bensidhoum@paris7.jussieu.fr

Institut National de la Santé et de la Recherche Médicale, Université Paris Sciences et Lettres, Centre National de la Recherche Scientifique, Paris, France

Hugues PoRTIER: hugues.portier@univ-orleans.fr

Institut National de la Santé et de la Recherche Médicale, Université Paris Sciences et Lettres, Centre National de la Recherche Scientifique, Paris, France

and

Collégium Sciences et Techniques, Université d'Orléans, Orléans, France

YVES RÉMOND: remond@unistra.fr

ICube Laboratory, Université de Strasbourg, Centre National de la Recherche Scientifique,

Strasbourg, France 


\title{
MODELS FOR DRUG RELEASE OF GENTAMICIN IN A POLYLACTIC ACID MATRIX
}

\author{
Anna S. Morozova, Elena N. Vilchevskaya, \\ Wolfgang H. MÜller ANd NikOlay M. Bessonov
}

\begin{abstract}
Recent experiments by Macha et al. (Front. Bioeng. Biotech. 7 (2019), art. id. 37) on the release of gentamicin embedded in a polylactic acid matrix film immersed in a body fluid solution have shown, first, a sudden burst phenomenon after several weeks and, second, a premature end to the release, such that a considerable amount of gentamicin is kept in the matrix. It is shown that such phenomena cannot be described adequately by assuming diffusion of the Fickian kind. In order to improve the modeling, extensions to Fickian diffusion are proposed as follows. The first one is of a phenomenological nature. A production term in the diffusion equation with intrinsic parameters is introduced, all of which can be interpreted intuitively and related to experimental data. The model allows one to capture the aforementioned departure from the timewise parabolic Fickian release characteristic eventually leading to complete release. Second, a micromodel is presented that provides a physical explanation for the proposed production: the drug is released from a carrier particle into the matrix, which eventually comes to an end due to the diminishing particle surface, and the drug adheres to a core due to surface tension. The material parameters of both models are determined by inverse analysis of experimental data.
\end{abstract}

\section{Introduction}

Polylactic acid (PLA) is one of the most common polymers used as a microcarrier of drugs [Jalil and Nixon 1990; Wischke and Schwendeman 2008; Conti et al. 1991]. Low toxicity, excellent biocompatibility, and the absence of inflammation in contact with living organisms, as well as good mechanical properties, make PLA attractive for the pharmaceutical industry, including for the creation of drugretarding systems [Conti et al. 1991].

It is important to predict drug release rates and to understand the primary processes that manage the release. Drug release from biodegradable polymeric carriers, such as a PLA matrix, mostly depends on loading efficiency of the drug,

\section{Communicated by Francesco dell'Isola.}

MSC2020: 60K50.

Keywords: diffusion parameter identification, drug release, diffusion models, finite volumes. 
solubility, biodegradability, diffusion, and the size of the carrier. Mathematical models are an important tool for the development of pharmaceutical compositions, the evaluation of drug release processes, and, in general, the optimal design of new systems [Peppas and Narasimhan 2014].

Significant work has been done on the mathematical modeling of release profiles. See, e.g., [Bruschi 2015, Chapter 5], according to which the most common mathematical models describing drug release are the zero-order model, the first-order model, the Higuchi model, the Hixson and Crowell model, and the KorsmeyerPeppas model.

In the zero-order model the release kinetics of the drug dissolution is only a function of time and the process takes place at a constant rate independent of the active substance concentration. This model holds true only in the case of very slow drug release.

The first-order model is based on the assumption that the change in concentration of drug in the drug carrier with respect to change in time depends only on its concentration and on the phenomenon of dissolution of a solid particle in a liquid with constant rate. The first-order model can be used to describe the drug dissolution in pharmaceutical dosage forms such as those containing soluble active substances incorporated in a porous matrix. For this system the amount of drug released is proportional to the amount of remaining drug in the matrix. Thus, the amount of active release tends to decrease as a function of time.

The Higuchi model [1963] was the first refined mathematical model that described the release of a drug from an insoluble matrix as a square-root-of-timedependent process based on Fickian diffusion. The model was initially derived for planar systems. However, since then it has been modified for use with different geometries and porous systems. This model is based on several assumptions: (i) the drug concentration in the matrix is initially much higher than the solubility of the drug, (ii) edge effects are negligible, so diffusion is unidirectional, (iii) the thickness of the dosage form is much larger than the size of the drug molecules, (iv) the swelling and dissolution of the matrix is negligible, (v) the diffusivity of the drug is constant, and (vi) perfect sink conditions are attained in the release environment.

The Hixson and Crowell model [1931] is used to describe the dissolution process of such dosage forms in which the dissolution surface decreases over time and the geometric shape itself remains the same. For example, this model can be used to describe the dissolution of a specimen consisting of identical spherical particles. This model is based on the assertion that the dissolution rate is proportional to the cube root of weight of the drug particles. When the Hixson and Crowell model is used, it is assumed that the drug release is limited by dissolution velocity and not by diffusion, which can occur through the polymeric matrix. 
The power law model developed in [Korsmeyer et al. 1983; Ritger and Peppas 1987] is a semiempirical equation describing drug release from polymeric systems. This law is useful when the release mechanism is not known or when more than one type of drug release phenomenon is involved. Depending on the value of the exponent of release that best matches the release profile of an active substance in a matrix system, it is possible to establish a classification according to the type of observed behavior: Fickian or non-Fickian models. In the Fickian model the drug release is governed by diffusion. In non-Fickian models the mechanisms driving the drug release are related to the relaxation of polymer chains, diffusion and swelling, or extreme forms of transport. The latter is characterized by tension and breaking of the polymer (solvent crazing).

All of these models can de used to describe the drug release of gentamicin (GM) in PLA carrier [Trang et al. 2019]. However, sudden burst phenomena are observed after several weeks on graphs of GM release from PLA microspheres, which the above models do not take into account. This kind of behavior was also observed in [Macha et al. 2019] during the initial release of GM from a PLA matrix, as well as a premature stop to the release.

Obviously different mathematical models are required to model such observations. To this end we will, in a first step, introduce a phenomenological production term in the classical Fickian diffusion equation, which contains two constitutive parameters for modeling the speed and extend of saturation in the two stages. In a second step, this production term will be introduced and interpreted based on microphysical considerations: the drug is embedded and needs to be delivered to the carrier first. However, the solubility of the embedded drug is limited. The effects resulting from the degradation of the polymer carrier will not be described here but studied in future work instead.

The two developed mathematical models will be tested on the experiment described by Macha et al. [2019], which we briefly summarize in the following passages. It should be noted that they also attempted to fit the data based on simple Fickian diffusion laws. Note that the models presented in this paper go considerably beyond this traditional approach.

In recent experiments by Macha et al. [2019] the dissolution behavior of different drugs in various carriers was investigated. One of the investigated systems concerned the release of GM in a PLA matrix as follows. As a release medium mimicking the body fluid, phosphate-buffered saline solution was used. This solution had a $\mathrm{pH}$ of 7.4 and was kept at a "body temperature" of $37 \pm 0.1^{\circ} \mathrm{C}$. The GM was stored in a nanoporous matrix made of PLA. A scanning electron micrograph (SEM) of the situation is shown in Figure 1. The PLA matrix is shown in gray color whereas GM blisters embedded within the matrix are in white. The distribution of the drug is fairly random. 


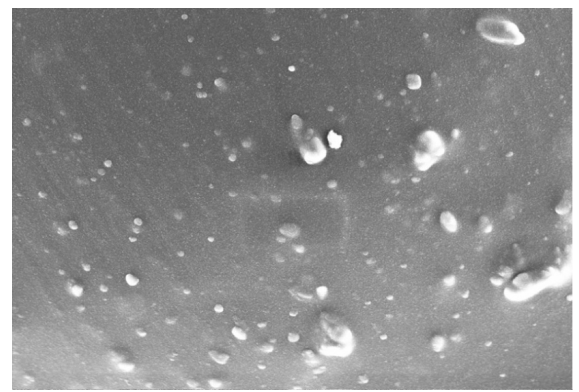

Figure 1. SEM of the GM containing PLA film.

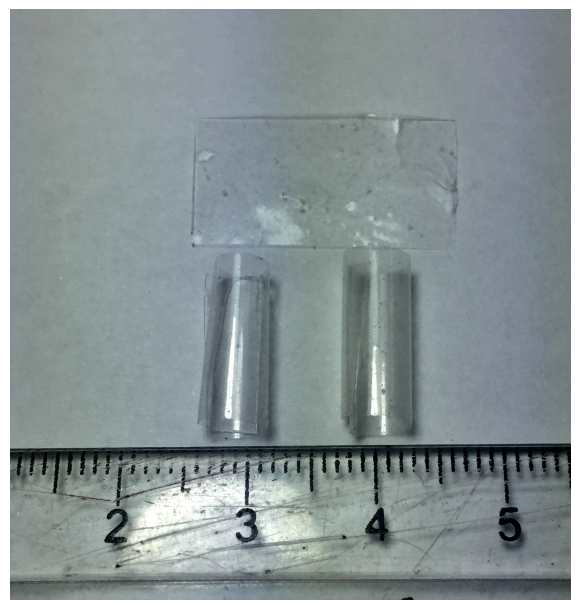

Figure 2. Film matrix specimens before and after curling.

In the experiment the drug-loaded PLA matrix sheet curled up and formed a cylindrical tube. After insertion into a tube filled with solution it slowly started to disintegrate. During this process more and more pore spaces for drug release within the PLA matrix opened up. The film matrix specimens before and after curling are shown in Figure 2.

The initial amount of drug in the matrix was $m_{\mathrm{d}}=7.5 \mathrm{mg}$, and the solution volume was $V_{\mathrm{s}}=15 \mathrm{ml}$. Therefore, the maximum expected drug release concentration is $c_{\max }=0.5 \mathrm{mg} / \mathrm{ml}$. This information was used to calculate the fraction of the drug released at time $t$,

$$
F(t)=\frac{c_{\mathrm{s}}(t)}{c_{\max }}, \quad 0 \leq F(t) \leq 1,
$$

where $c_{\mathrm{s}}(t)=m(t) / V_{\mathrm{s}}$ is the average concentration of the drug in the solution at time $t$. 


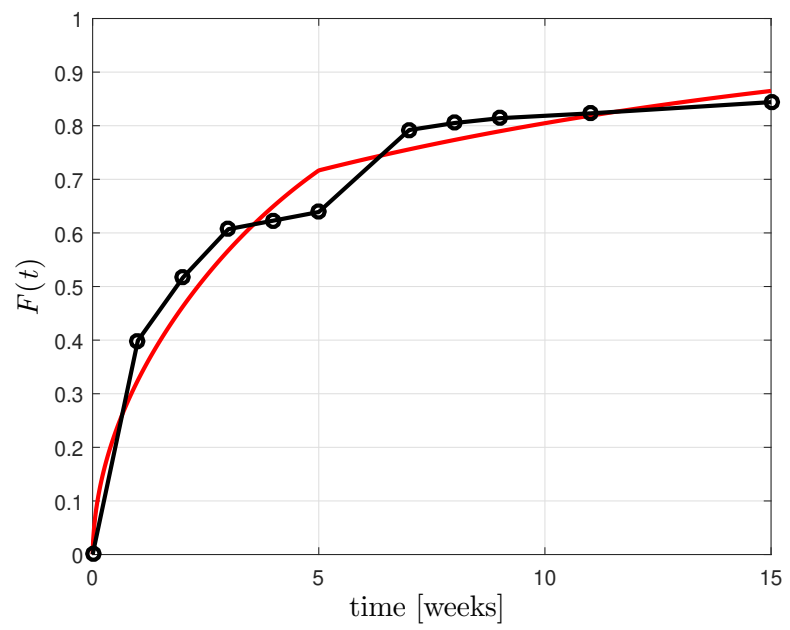

Figure 3. Fractional cumulative release for GM in PLA.

The dependence of the experimentally determined fractional cumulative release on time is indicated by the black circles in Figure 3.

The curve of Figure 3 has several characteristic features. We can clearly distinguish two stages, before and after the fifth week. Moreover, between weeks 1 and 3 the curve runs initially in a parabolic fashion, which indicates Fickian diffusion behavior. However, between weeks 3 and 5 it levels off as if supply of drug is lacking. After that between weeks 5 and 7 we observe another steep boost, which if considered as Fickian would result in a parabolic growth law in time leading to a $100 \%$ dissolution of drug in the body fluid. However, in reality this is not so and again some saturation can be observed. Indeed, the connecting black lines during weeks 5 to 15 clearly indicate a nonparabolic, i.e., non-Fickian behavior.

To demonstrate this quantitatively, an inverse analysis was performed based on a purely Fickian model (similar as in [Macha et al. 2019] or [Rickert et al. 2019], where also more details regarding the computational methods can be found). From the experiment it is known that the matrix curls into a tube. Therefore, we consider a hollow cylinder $\Omega$ of internal and outer radii $r_{\mathrm{i}}=3.2 \mathrm{~mm}$ and $r_{\mathrm{o}}=3.4 \mathrm{~mm}$, respectively, with a height of $H=20 \mathrm{~mm}$; see Figure 4. Since the thickness $d=$ $r_{\mathrm{o}}-r_{\mathrm{i}}=0.2 \mathrm{~mm}$ of the cylinder is very small, it is assumed that the drug is released only through the inner and outer side surfaces $\Gamma$ and not through the rims at the top into the body fluid solution. The distribution in the $z$ direction is assumed to be uniform. In addition it is assumed that compared to the matrix the diffusion of the drug in the body fluid is much faster and a homogeneous distribution is instantaneously assumed. No differential equations need to be used to analyze that process. We assume that all that is released from the matrix into the body fluid 


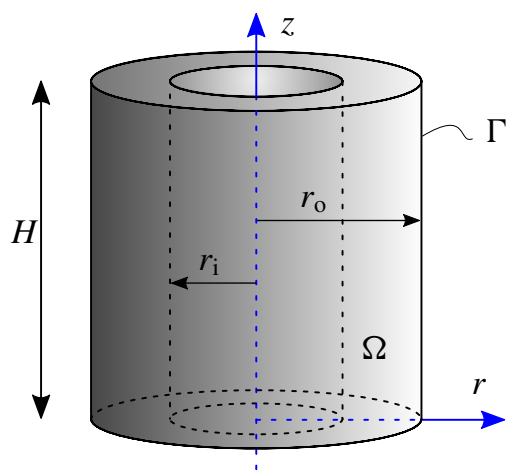

Figure 4. Cylindrical geometry.

is immediately homogeneously distributed in there and contributes to $F(t)$, which can then be calculated by simple algebra. Our goal is to describe the diffusion process within the matrix domain and the mass transfer across the boundary, as well as to determine the diffusion coefficient of the dissolved drug in the matrix, which is one of the key parameters characterizing the kinetics of release. To this end one may think simplistically of using the mass balance together with Fick's first law,

$$
\frac{\partial c(r, t)}{\partial t}=-\nabla \cdot \boldsymbol{J}, \quad \boldsymbol{J}=-D \nabla c(r, t),
$$

in order to obtain the classical diffusion equation, which describes the diffusion process in the matrix $\mathrm{m}$,

$$
\frac{\partial c_{\mathrm{m}}(r, t)}{\partial t}=D \Delta c_{\mathrm{m}}(r, t) \equiv\left(\frac{\partial^{2}}{\partial r^{2}}+\frac{1}{r} \frac{\partial}{\partial r}\right) c_{\mathrm{m}}(r, t) \quad \text { for all } r \in \Omega,
$$

$c_{\mathrm{m}}$ being the concentration of drug in the matrix, which depends on both time and position, and an equation (or boundary/transfer condition) describing the mass transfer across the boundary into the solution $\mathrm{s}$,

$$
V_{\mathrm{s}} \frac{\partial c_{\mathrm{s}}(t)}{\partial t}=\left.2 \pi H D\left(r \frac{\partial c_{\mathrm{m}}(r, t)}{\partial r}\right)\right|_{\Gamma},
$$

where $V_{\mathrm{S}}$ denotes the volume of the solution of body fluid and $c_{\mathrm{S}}(t)$ is the timedependent concentration in the solution.

As initial conditions, a uniform distribution of the drug in the matrix and the absence of the drug in the solution are assumed: $c_{\mathrm{m}}(r, t=0)=c_{0}$ for all $r \in \Omega$ and $c_{\mathrm{S}}(t=0)=0$, where $c_{0}$ is the initial concentration of drug in the matrix,

$$
c_{0}=\frac{m_{\mathrm{d}}}{H \pi\left(r_{\mathrm{o}}^{2}-r_{\mathrm{i}}^{2}\right)} \approx 90.4 \mathrm{mg} / \mathrm{ml} .
$$


The equations of the model were solved numerically by using the finite volume method [Macha et al. 2019; Rickert et al. 2019]. In order to account for the two stages, two corresponding diffusion coefficients were introduced.

The diffusion coefficients for two different stages were determined by minimizing the target function $E=(1 / N) \sum_{i=0}^{N}\left|\left(F_{\exp }\left(t_{i}\right)-F_{\text {num }}\left(t_{i}\right)\right) / F_{\text {num }}\left(t_{i}\right)\right|$, where $F_{\text {exp }}$ refers to the experimentally determined data known at discrete times $t_{i}$ with $i \in[0,10]$. Moreover, $F_{\text {num }}=c_{s}(t) / c_{\max }$ is the numerically predicted function.

The mass release is depicted in Figure 3 by the red curve. The resulting values of the diffusion coefficients for the two stages, (I) $t \in[0,5]$ weeks and (II) $t \in[5,15]$ weeks, are

$$
D_{\mathrm{I}}=1.461 \times 10^{-9} \mathrm{~mm}^{2} / \mathrm{s}, \quad D_{\mathrm{II}}=5.183 \times 10^{-10} \mathrm{~mm}^{2} / \mathrm{s} .
$$

Note that the values differ only slightly from those shown in [Rickert et al. 2019, (12.47)] from a finite element discretization. The following remarks are in order: (i) In stage I the prediction first underestimates the observed release and then begins to take off. There is no leveling off or saturation. (ii) The release prediction in stage II first overshoots a little and then begins to take off. Again it does not allow for a saturation below $100 \%$ of release. It would continue to increase and finally get close to $100 \%$ of cumulative release. (iii) The predicted diffusion coefficient during stage II is less than that of stage I. This is contrary to intuition, because we believe the matrix will start to deteriorate with ongoing time, which creates fissures and, therefore, phenomenologically speaking, the diffusion coefficient should increase. In summary, it is fair to say that this approach is more or less a brute-force fit ignoring possible physical effects based on the simplest type of diffusion equations available. We proceed to improve the situation.

\section{Advanced modeling process}

\subsection{Model 1: a phenomenological production term mimicking a locking effect.}

The main feature of this model is that it allows only a limited precipitation of the drug in the carrier matrix to the solution. It is achieved by adding a phenomenologically introduced sink (or negative production) term $-\chi(r, t)$. Its purpose is to mimic a locking effect in the matrix so that after a certain time $t^{*}$ it will stop the release of the drug. Consequently, we write

$$
\frac{\partial c_{\mathrm{m}}(r, t)}{\partial t}=D \Delta c_{\mathrm{m}}(r, t)-\chi(r, t) \quad \text { for all } r \in \Omega,
$$

while (4), describing the mass transfer across the boundary and the initial conditions, remains unchanged. 

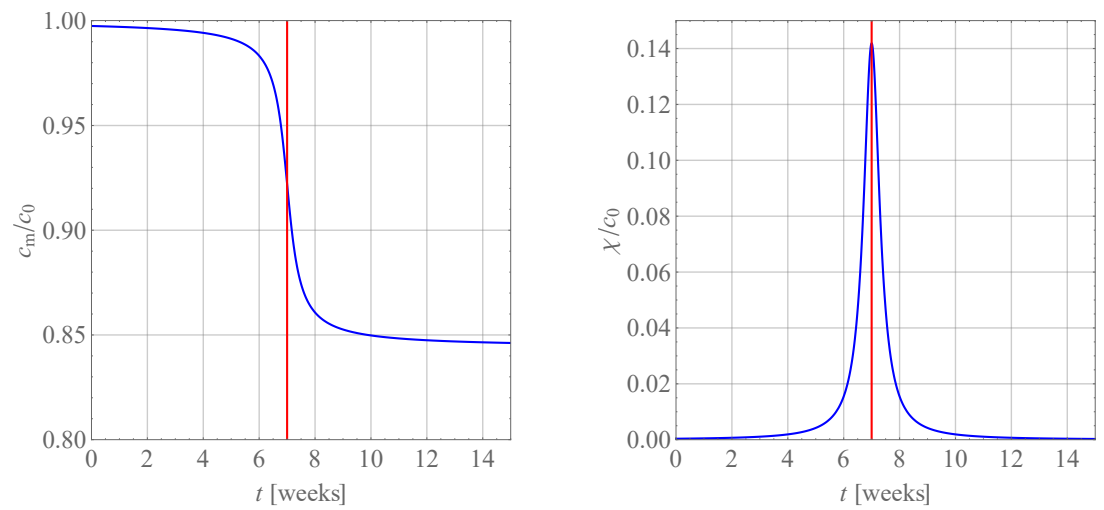

Figure 5. Matrix concentration (9) and production term (11) as a function of time. Time $t_{*}$ is indicated by a red line.

In order to find an explicit expression for $\chi(r, t)$ we first consider (7) without the flux term, i.e.,

$$
\frac{\partial c_{\mathrm{m}}(r, t)}{\partial t}=-\chi(r, t) .
$$

A suitable form of the production is then found by means of a phenomenological argument: we want the concentration of the drug at every point in the matrix to change around a certain point in time, $t_{*}=7$ weeks, from the level $\tilde{c}_{\mathrm{m}}^{*}$, and then finally assumes a constant level $\tilde{c}_{\mathrm{m}}^{\infty}$, where the symbol " $\infty$ " refers to long times, namely 15 weeks. This behavior can be described by using the function

$$
c_{\mathrm{m}}(r, t)=\frac{\tilde{c}_{\mathrm{m}}^{*}-\tilde{c}_{\mathrm{m}}^{\infty}}{\pi} \arctan \left(-\frac{t-t_{*}}{\varepsilon t_{*}}\right)+\frac{\tilde{c}_{\mathrm{m}}^{*}+\tilde{c}_{\mathrm{m}}^{\infty}}{2},
$$

where $\varepsilon$ is a dimensionless parameter allowing us to control the slope of the curve. We can estimate all parameters from the experimental release profile at $t_{*}=7$ and $t_{\infty}=\infty \approx 15$ weeks, i.e., the beginning and the saturation of the second stage:

$$
\tilde{c}_{\mathrm{m}}^{*}=1 \times c_{0}, \quad \tilde{c}_{\mathrm{m}}^{\infty}=0.844 \times c_{0} .
$$

Moreover, in order to guarantee the observed transition sharpness, we choose $\varepsilon=0.05$. The concentration-time function is shown on the left of Figure 5. The transition into saturation is clearly visible.

Then by substituting this function into (8), we obtain the following expression for the production term:

$$
\chi(r, t)=\frac{\tilde{c}_{\mathrm{m}}^{*}-\tilde{c}_{\mathrm{m}}^{\infty}}{\pi \varepsilon t_{*}}\left(1+\left(\frac{t_{*}-t}{\varepsilon t_{*}}\right)^{2}\right)^{-1} .
$$


The production term is shown on the right of Figure 5. As it should be, it is a very sharp function distinctly different from zero only around the time $t_{*}$, which is indicated by the straight red lines in the plots. This also means that there is nearly no production during stage I. Here the release process will essentially be modeled as Fickian.

\subsection{Model 2: production term obtained from a micromodel. Note that Model 1} of Section 2.1 was phenomenological in the sense that we made the drug release stop by prescribing an appropriate production term. In this subsection we attempt to give reasons why the release stops and will "derive" the corresponding production term from micromechanical considerations. To this end, we will assume that initially the matrix as well as the solution do not contain any drug, i.e., $c_{\mathrm{m}}(r, t=0)=0$ for all $r \in \Omega$ and $c_{\mathrm{s}}(t=0)=0$, but rather the matrix will be filled gradually with drug by dissolution of the drug particles stored within. As in (7) of Section 2.1 this process is described by an additional (this time positive) production term $\chi(r, t)$ in the diffusion equation,

$$
\frac{\partial c_{\mathrm{m}}(r, t)}{\partial t}=D \Delta c_{\mathrm{m}}(r, t)+\chi(r, t) \quad \text { for all } r \in \Omega,
$$

and (4) describing the mass transfer across the boundary remains unchanged. The diffusion coefficient $D$ is unknown and will be determined from inverse analysis.

In order to obtain a suitable form for a physics-based production term we now argue microscopically and start from a dissolution law established by Shukarev [Zelikman et al. 1983, p. 424; Wikipedia 2017] to obtain its concrete mathematical form. This law is formulated as follows. The amount of substance transferred across the interface by dissolution of a single particle is proportional to the difference between the concentration at the interface $\left.c\right|_{\widetilde{\Gamma}}$ and the concentration further away, in other words by the "intensity of the sensing phase" $c$, the phase contact surface $\mathrm{d} F$, and time increment $\mathrm{d} t$ :

$$
\mathrm{d} m=\alpha(c \mid \widetilde{\Gamma}-c) \mathrm{d} F \mathrm{~d} t,
$$

where $\mathrm{d} m$ is the mass increment of the solute and $\alpha$ the mass transfer coefficient.

Now in order to get to the continuum scale we homogenize and assume that the drug-carrying particles are all spherical of the same initial radius $R_{0} \approx 7.5 \times 10^{-6} \mathrm{~m}$ based on SEM examinations. For simplicity and in a first step it is also assumed that they are initially uniformly distributed throughout the matrix. This means that the particle density $n=N / V_{\mathrm{m}}$ is constant, while $N$ is the number of particles and $V_{\mathrm{m}}$ is a representative volume element of the matrix. Then Shukarev's law suggests the following form for the production term on the continuum level:

$$
\chi(r, t)=4 \pi R^{2}(t) \alpha(R) n\left[c_{\mathrm{m}} \mid \widetilde{\Gamma}-c_{\mathrm{m}}(r, t)\right] \text { for all } r \in \Omega,
$$


where $R(t)$ is the particle radius, which is a function of time, $n=N / V_{\mathrm{m}}$ is the particle number density, $N$ is the number of particles, $V_{\mathrm{m}}$ is the matrix volume, and $c_{\mathrm{m}} \mid \widetilde{\Gamma}$ is the concentration of dissolved drug at the interface between solid drug and dissolved drug. It will be estimated from the condition that the part of the drug that can dissolve in the remaining volume of the matrix is completely dissolved:

$$
c_{\mathrm{m}} \mid \widetilde{\Gamma}=\frac{N \cdot \rho_{\mathrm{d}} \cdot \frac{4}{3} \pi\left(R_{0}^{3}-R_{\mathrm{cr}}^{3}\right)}{V_{\mathrm{m}}-N \cdot \frac{4}{3} \pi R_{\mathrm{cr}}^{3}},
$$

where $\rho_{\mathrm{d}}=1032 \mathrm{mg} / \mathrm{ml}$ is the mass density of drug and $R_{\mathrm{cr}}$ is a critical radius to which the drug particles dissolve and which will be discussed further. And $\alpha(R)$ is the mass transfer coefficient, which depends on the radius of the drug particle.

We proceed to give a reason why the mass transfer coefficient is not constant but is rather a kinetic characteristic. We assume that it is a function of the (average homogenized) radius $R$ of the drug particles. In fact, the smaller the radius of the particle, the greater the surface tension and therefore the smaller the mass transfer coefficient. We must also take into account that the drug particles do not completely dissolve, i.e., a certain critical radius $R_{\mathrm{cr}}$ exists. When the particle approaches this radius, the production stops, because the stabilizing surface tension is too strong. This fact has been known for a long time [Finholt and Solvang 1968] and observed until today [Dahlberg et al. 2008]. Hence, we propose the following linear approximation carrying all these features:

$$
\alpha(R)=\alpha_{0} \frac{R(t)-R_{\mathrm{cr}}}{R_{0}-R_{\mathrm{cr}}},
$$

where $\alpha_{0}$ is an amplitude to be determined from inverse analysis. As evident by its unit it is characteristic of the speed of dissolution. The critical radius $R_{\mathrm{cr}}$ is a parameter to be adjusted depending on the observed saturation level and can be calculated from mass conservation:

$$
[1-F(15 \text { weeks })] m_{0}=N \cdot \rho_{\mathrm{d}} \cdot \frac{4}{3} \pi R_{\mathrm{cr}}^{3} \Longrightarrow R_{\mathrm{cr}} \approx 0.54 R_{0}, c_{\mathrm{m}} \mid \widetilde{\Gamma} \approx 74 \mathrm{mg} / \mathrm{ml}
$$

In other words, the parameter $R_{\text {cr }}$ puts an end to the dissolution. As mentioned before the idea is that surface tension limits the size of particles that can dissolve. Clearly, the particle radius will change with time until it reaches this limit. Hence, we need a suitable kinetic equation. If we consider the dissolution of one drug particle, Shukarev's law reads

$$
\dot{m}(t)=-\alpha(R)\left[c_{\mathrm{m}} \mid \widetilde{\Gamma}-c_{\mathrm{m}}(r, t)\right] \cdot 4 \pi R^{2}(t) .
$$

We decompose the rate of change of drug mass as a product of drug density and the rate of change in particle volume:

$$
\rho_{\mathrm{d}} \dot{V}(t)=-\alpha(R)\left(c_{\mathrm{m}} \mid \widetilde{\Gamma}-c_{\mathrm{m}}(r, t)\right) \cdot 4 \pi R^{2}(t) .
$$


After substituting the volume of the spherical particle $V(t)=4 / 3 \pi R^{3}(t)$ into (19) we get

$$
\rho_{\mathrm{d}} \frac{\mathrm{d} R(t)}{\mathrm{d} t}=-\alpha(R)\left[c_{\mathrm{m}} \mid \widetilde{\Gamma}-c_{\mathrm{m}}(r, t)\right]
$$

Hence, during the inverse analysis we must solve the coupled system of equations (7), (14), (16), and (20), subject to the initial and boundary conditions mentioned at the beginning of this subsection and $R(0)=R_{0}$.

\section{Results and discussion}

We begin by presenting the results of the phenomenological model with the negative production from Section 2.1. The predicted mass release is shown in Figure 6. Two values for the diffusion coefficients during the two stages were obtained from inverse analysis of the data:

$$
D_{\mathrm{I}}=1.470 \times 10^{-9} \mathrm{~mm}^{2} / \mathrm{s}, \quad D_{\mathrm{II}}=1.509 \times 10^{-9} \mathrm{~mm}^{2} / \mathrm{s} .
$$

Note that $D_{\text {II }}$ is slightly larger than $D_{\text {I }}$. Coincidentally this is confirms our intuition: we expect fissures in the matrix to be generated and to increase during longer exposure of the specimen to the body fluid. Overall this would correspond to an increasing diffusion coefficient. However, it should be emphasized that this result is nothing more than a coincidence and not really substantiated by the model.

Summarizing we may say that the traditional Fickian model of drug release from the matrix and its release into a body fluid solution was extended by introducing a phenomenologically motivated production term. It turns out that the stagnation of the release can be modeled adequately in the second stage during weeks 5 to 15 .

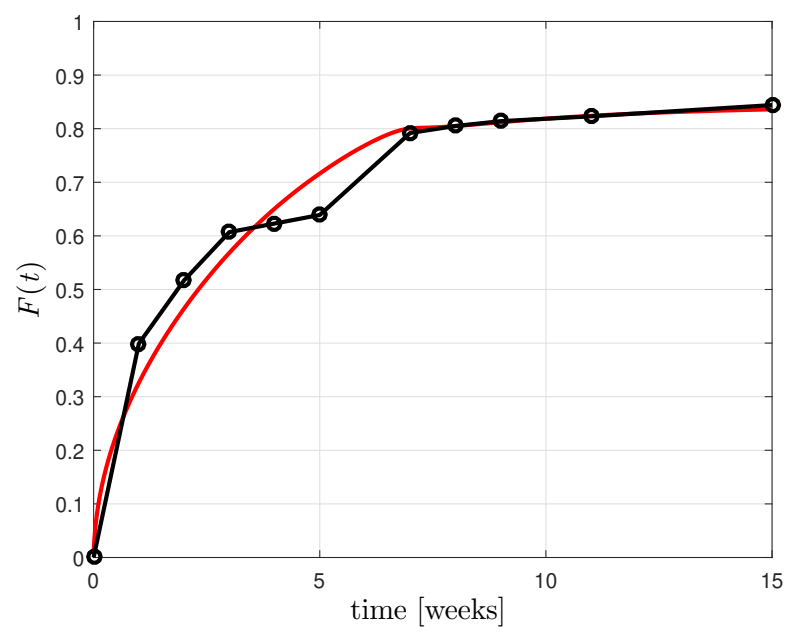

Figure 6. Fractional cumulative release with two-stage diffusion coefficient. 


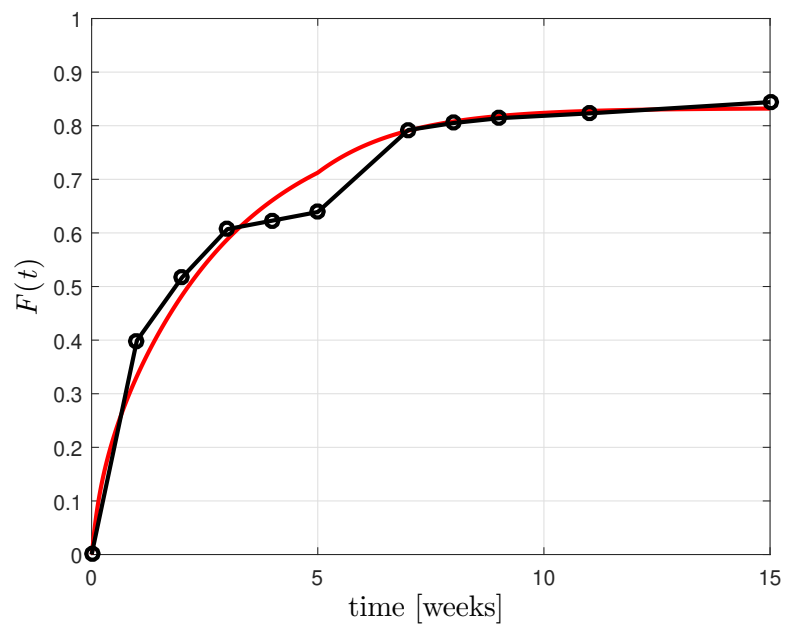

Figure 7. Fractional cumulative release predicted by Shukarev's dissolution model.

We now turn to the micromechanical model with constant particle distribution in the matrix from Section 2.2. The predicted mass release and the experimental data are depicted in Figure 7.

The corresponding values for the diffusion coefficients during the two stages are

$$
D_{\mathrm{I}}=2.418 \times 10^{-9} \mathrm{~mm}^{2} / \mathrm{s}, \quad D_{\mathrm{II}}=3.588 \times 10^{-9} \mathrm{~mm}^{2} / \mathrm{s} .
$$

As part of the inverse analysis the following value for the model parameter of the mass transfer coefficient (16) was obtained: $\alpha_{0}=2 \times 10^{-9} \mathrm{~m} / \mathrm{s}$.

By looking at the graph it turns out that the stagnation of the release can be modeled quite well in the second stage during weeks 5 to 15 . As in the model from Section 2.1, the first stage stagnation after week 3 is not represented at all. Again the diffusion coefficient for the second stage is slightly larger than during the first stage. As discussed before this agrees conveniently with our intuitive view for easier diffusion due to progressing deterioration of the matrix. It is to be understood that the diffusion coefficient is a kinematic characteristic that, generally speaking, depends on many facts (including the ones listed above). Therefore, our assumption about the relationship between the quality of the matrix and the values of the diffusion coefficient has been put into a new perspective.

\section{Conclusions and outlook}

Various mathematical models have been analyzed in order to describe the diffusion process in a carrier matrix and drug release across the boundary. After a short review of results from classical Fickian diffusion an extended diffusion equation 
with two different production terms was presented. These can mimic a locking effect, one on a phenomenological basis and the other one on microscopic grounds regarding the dissolution of individual drug particles.

It is intuitively clear that the deterioration of the quality of the polymer matrix over time can be "incorporated" in the determination of the diffusion coefficient for two different stages - before and after the fifth week.

However, we must conclude that the suggested models can give only an approximate understanding of the drug release described in [Macha et al. 2019], since they do not describe the saturation during the first phase as well as the destruction of the carrier polymer matrix into parts before the active substance is completely released, which is one of the reasons for the burst-type release. The analysis of this is left to future research.

Moreover, our enhanced models may well describe drug dissolution and diffusion in a nonbiodegradable polymer matrix. Such a matrix after the release of the active substance must be removed from the body either mechanically or through the gastrointestinal tract. To describe the release of a drug from such a matrix, we do not need to introduce two stages and two diffusion coefficients.

\section{References}

[Bruschi 2015] M. L. Bruschi, Strategies to modify the drug release from pharmaceutical systems, Woodhead Publishing Series in Biomedicine 85, Woodhead, Cambridge, UK, 2015.

[Conti et al. 1991] B. Conti, F. Pavanetto, and I. Genta, "Use of polylactic acid for the preparation of microparticulate drug delivery systems", J. Microencapsul. 9:2 (1991), 153-166.

[Dahlberg et al. 2008] C. Dahlberg, A. Millqvist-Fureby, and M. Schuleit, "Surface composition and contact angle relationships for differently prepared solid dispersions", Eur. J. Pharm. Biopharm. 70:2 (2008), 478-485.

[Finholt and Solvang 1968] P. Finholt and S. Solvang, "Dissolution kinetics of drugs in human gastric juice: the role of surface tension", J. Pharm. Sci. 57:8 (1968), 1322-1326.

[Higuchi 1963] T. Higuchi, "Mechanism of sustained-action medication: theoretical analysis of rate of release of solid drugs dispersed in solid matrices", J. Pharm. Sci. 52:12 (1963), 1145-1149.

[Hixson and Crowell 1931] A. W. Hixson and J. H. Crowell, "Dependence of reaction velocity upon surface and agitation", Ind. Eng. Chem. 23:8 (1931), 923-931.

[Jalil and Nixon 1990] R. Jalil and J. R. Nixon, "Biodegradable poly(lactic acid) and poly(lactide-coglycolide) microcapsules: problems associated with preparative techniques and release properties", J. Microencapsul. 7:3 (1990), 297-325.

[Korsmeyer et al. 1983] R. W. Korsmeyer, R. Gurny, E. Doelker, P. Buri, and N. A. Peppas, "Mechanisms of solute release from porous hydrophilic polymers", Int. J. Pharm. 15:1 (1983), 25-35.

[Macha et al. 2019] I. J. Macha, B. Ben-Nissan, E. N. Vilchevskaya, A. S. Morozova, B. E. Abali, W. H. Müller, and W. Rickert, "Drug delivery from polymer-based nanopharmaceuticals: an experimental study complemented by simulations of selected diffusion processes", Front. Bioeng. Biotech. 7 (2019), art. id. 37. 
[Peppas and Narasimhan 2014] N. A. Peppas and B. Narasimhan, "Mathematical models in drug delivery: How modeling has shaped the way we design new drug delivery systems", J. Control. Release 190 (2014), 75-81.

[Rickert et al. 2019] W. Rickert, A. Morozova, W. H. Müller, E. N. Vilchevskaya, B. Ben-Nissan, and I. Macha, "Drug delivery from polymer-based nanopharmaceuticals: simulations of selected diffusion processes", Chapter 12, pp. 207-226 in Contributions to advanced dynamics and continuum mechanics, edited by H. Altenbach et al., Advanced Structured Materials 114, Springer, 2019.

[Ritger and Peppas 1987] P. L. Ritger and N. A. Peppas, "A simple equation for description of solute release, I: Fickian and non-Fickian release from non-swellable devices in the form of slabs, spheres, cylinders or discs", J. Control. Release 5:1 (1987), 23-36.

[Trang et al. 2019] T. T. T. Trang, M. Mariatti, H. Y. Badrul, K. Masakazu, X. T. T. Nguyen, and A. A. H. Zuratul, "Drug release profile study of gentamicin encapsulated poly(lactic acid) microspheres for drug delivery", Mater. Today Proc. 17 (2019), 836-845.

[Wikipedia 2017] "Sink condition", Wikipedia entry, 2017, Available at https://en.wikipedia.org/ wiki/Sink_condition?oldid=758830177.

[Wischke and Schwendeman 2008] C. Wischke and S. P. Schwendeman, "Principles of encapsulating hydrophobic drugs in PLA/PLGA microparticles”, Int. J. Pharm. 364:2 (2008), 298-327.

[Zelikman et al. 1983] A. Zelikman, G. Voldman, and L. Belyaevskaya, Theory of hydrometallurgical processes, Metallurgiya, Moscow, 1983. In Russian.

Received 30 Apr 2020. Revised 6 Jul 2020. Accepted 28 Sep 2020.

AnNA S. Morozova: aveeanka@gmail.com

Peter the Great Saint Petersburg Polytechnic University, Saint Petersburg, Russia

ELENA N. VILCHEVSKAYA: vilchevskaya_en@spbstu.ru

Institute for Problems in Mechanical Engineering of the Russian Academy of Sciences,

Saint Petersburg, Russia

WOLFGANG H. MüLLER: wolfgang.h.mueller@tu-berlin.de

Fachgebiet Kontinuumsmechanik und Materialtheorie, Institut für Mechanik,

Technische Universität Berlin, Berlin, Germany

NiKOLAY M. BESSONOV: nickbessonov1@gmail.com

Institute for Problems in Mechanical Engineering of the Russian Academy of Sciences,

Saint Petersburg, Russia 


\title{
ANALYTICAL MECHANICS ALLOWS NOVEL VISTAS ON MATHEMATICAL EPIDEMIC DYNAMICS MODELING
}

\author{
PAUL STEINMANN
}

\begin{abstract}
This contribution aims to shed light on mathematical epidemic dynamics modeling from the viewpoint of analytical mechanics. To set the stage, it recasts the basic SIR model of mathematical epidemic dynamics in an analytical mechanics setting. Thereby, it considers two possible reparametrizations of the basic SIR model: one rescales time, while the other transforms the coordinates, i.e., the independent variables. In both cases, Hamilton's equations in terms of a suited Hamiltonian as well as Hamilton's principle in terms of a suited Lagrangian are considered in minimal and extended phase and state space coordinates, respectively. The corresponding Legendre transformations relating the various options for the Hamiltonians and Lagrangians are detailed. Ultimately, this contribution expands on a multitude of novel vistas on mathematical epidemic dynamics modeling that emerge from the analytical mechanics viewpoint. As a result, it is believed that interesting and relevant new research avenues open up when exploiting in depth the analogies between analytical mechanics and mathematical epidemic dynamics modeling.
\end{abstract}

\section{Motivation}

The global COVID-19 pandemic, with alleged outbreak by the end of 2019 in Wuhan, China [Lu et al. 2020] - despite its devastating implications for health, economy, and society - has in particular challenged modeling and simulation of mathematical epidemic dynamics. Political decision makers around the globe seek (or should seek) advice from scientists such as virologists, biologists, clinicians, economists, and sociologists as well as modelers from different fields. Especially the latter are in the position to virtually simulate various scenarios based on wellfounded assumptions in order to provide support and guidance for the difficult and momentous decisions of politicians, e.g., on lockdown measures and stepwise exit strategies thereof. Thus, the critical importance of modeling is clearly appreciated, and indeed, mathematical epidemic dynamics modeling is a well-established and mature field.

\section{Communicated by Francesco dell'Isola.}

MSC2020: 70K99.

Keywords: epidemics modeling, analytical mechanics. 
Traditional mathematical modeling of epidemic dynamics is rooted in the concept of susceptible, infected, and recovered (SIR) compartments as originally proposed in [Kermack and McKendrick 1927; 1932; 1933; 1937; 1939]. Various modifications extend the classical SIR model to account for further compartments such as, e.g., deceased (SIRD model), exposed (SEIR model), and quarantined (SIQRD model), among many other sophisticated options [Hethcote 2000; Diekmann et al. 2013]. Classical SIR-type compartment-based models are coupled ordinary differential equations (ODEs). Extending the ODE-based SIR-type modeling approach to integro-differential equations (IDEs) allows one to also consider the detailed course of the disease, e.g., delay due to incubation time and the infectious period [Keimer and Pflug 2020]. SIR-type models describe the temporal spread of infectious diseases for integral populations, thereby, however, neglecting the interconnectedness of spatially distributed geographic areas. Recently proposed multiple compartment models, e.g., the mcSIR model in [Seroussi et al. 2019], also allow consideration of the geographical spread of potentially multiple infectious virus strains within the population and its potentially multiple subgroups (e.g., age groups). Spatial network models, e.g., [Balcan et al. 2009; 2010; Pastor-Satorras et al. 2015], for example based on the SEIR model at each network node can qualitatively simulate the outbreak dynamics of infectious diseases and the impact of travel restrictions in geographical areas at the global (macro) scale such as China and the USA [Peirlinck et al. 2020] or Europe [Linka et al. 2020]. However, due to its stochastic nature and strong impact of socio-economic factors, modeling epidemic dynamics within geographical areas at the local (micro) scale requires the use of another modeling paradigm, i.e., rule-driven, agent-based models [Rahmandad and Sterman 2008]. Agent-based models allow for example studying the effect of various lockdown exit strategies on local geographical entities with only a comparatively small number of individuals (agents); see, e.g., [German et al. 2020]. Regardless of the modeling approach taken, quantitative predictions of epidemic dynamics remain challenging and critically require careful identification of model parameters from reliable databases; see, e.g., [Kergaßner et al. 2020b].

Given this mature state of affairs, why is a novel, alternative view on mathematical epidemic dynamics modeling justified at all?

The answer is as follows: to date, modelers of complex mechanical systems and behavior have developed a versatile and extremely successful toolset, including sophisticated analytical and in particular efficient and accurate computational methods. Examples are techniques to master severe nonlinearities and couplings with nonmechanical fields, a multitude of multiscale and homogenization modeling approaches as well as incorporating uncertainty quantification into modeling and simulation. Mathematical epidemic dynamics modeling can undoubtedly benefit 
largely from this accumulated expertise! In summary, it is therefore believed that first translating epidemic dynamics models into an analytical mechanics setting (related steps towards this aim may be found, e.g., in [Militaru and Munteanu 2013; Ionescu et al. 2015; Seroussi et al. 2019]) and then, secondly, exploiting the analogy between the two approaches while utilizing the full toolset of mechanical modeling can provide novel vistas and unprecedented opportunities. The present contribution aims to sketch out a few of these perspectives and to encourage the mechanics community to offer its strong modeling expertise to possibly and hopefully help further improve epidemic dynamics modeling.

\section{Basic SIR model}

Classical modeling of epidemics dynamics is rooted in the concept of susceptible, infected, and recovered (SIR) compartments as originally proposed in [Kermack and McKendrick 1927; 1932; 1933; 1937; 1939]. The basic compartment-based SIR model is the set of two coupled ordinary differential equations (ODEs)

$$
\left[\begin{array}{l}
I^{\bullet} \\
S^{\bullet}
\end{array}\right]=\left[\begin{array}{c}
\beta S I-\gamma I \\
-\beta S I
\end{array}\right]
$$

Here, $I$ and $S$ denote the stock of individuals in the infected and the susceptible compartments, respectively, normalized by the size of the entire population. The notation for the derivative of a quantity with respect to ordinary time $t$ is

$$
\{\cdot\}^{\bullet}:=\mathrm{d}_{t}\{\cdot\} .
$$

The parameters $\beta$ and $\gamma$ are the infection and the recovery rate, respectively, with their ratio defining the basic reproduction number $R_{0}:=\beta / \gamma$. Note finally that the stock of individuals in the recovered compartment follows from the constraint $S+I+R=1$; thus, the evolution equation $R^{\bullet}=-\left[S^{\bullet}+I^{\bullet}\right]$ is tacitly suppressed in our presentation.

\section{Time reparametrized SIR model}

In order to recast the basic SIR model into a format more amenable to the analytical mechanics setting, it is proposed, as a first option, to rescale time as

$$
\tau:=\int_{0}^{t} S I \mathrm{~d} \bar{t} \quad \text { with } \quad \mathrm{d} \tau / \mathrm{d} t=S I .
$$

The notation for the derivative of a quantity with respect to rescaled time $\tau$ is

$$
\{\cdot\}^{\circ}:=\mathrm{d}_{\tau}\{\cdot\} \text {. }
$$


Consequently, the derivatives with respect to rescaled and ordinary time are related via

$$
\{\cdot\}^{\circ}=\{\cdot\}^{\bullet} /[S I] .
$$

As a result, the basic SIR model is eventually reparametrized in terms of rescaled time as

$$
\left[\begin{array}{l}
I^{\circ} \\
S^{\circ}
\end{array}\right]=\boldsymbol{F} \quad \text { with } \quad \boldsymbol{F}:=\left[\begin{array}{c}
\beta-\gamma / S \\
-\beta
\end{array}\right]=:\left[\begin{array}{l}
V \\
F
\end{array}\right] .
$$

In the time reparametrized SIR model the right-hand side is abbreviated as the forcing term $\boldsymbol{F}$, i.e., as the column matrix consisting of the time reparametrized rate of infection $V$ and force of infection $F$ (a common terminology from mathematical epidemiology already establishing a semantic analogy to mechanics). Computing $I^{\circ \circ}=\left[\gamma / S^{2}\right] S^{\circ}$ from $(6)_{1}$ and eliminating $S^{\circ}$ with the help of $(6)_{2}$ renders $I^{\circ \circ}=$ $-\beta \gamma / S^{2}$. Resorting finally again to $(6)_{1}$, i.e., expressing $S\left(I^{\circ}\right)=\gamma /\left[\beta-I^{\circ}\right]$, allows formulating the time reparametrized SIR model as a single nonlinear ODE

$$
I^{\circ \circ}=-R_{0}\left[\beta-I^{\circ}\right]^{2}
$$

exclusively in the stock of individuals in the infected compartment and with the basic reproduction number and the infection rate as parameters.

3.1. Hamiltonian in minimal phase space coordinates. The minimal phase space coordinates collectively assembled in the column matrix $\boldsymbol{Z} \in \mathbb{R}^{2}$, i.e., the generalized coordinate $Q$ defined as the stock of individuals in the infected compartment and the generalized momentum $P$ defined as the stock of individuals in the susceptible compartment, span the two-dimensional phase space $\mathbb{P}$; thus,

$$
\mathbb{P}:=\left\{\boldsymbol{Z}:=\left[\begin{array}{l}
Q \\
P
\end{array}\right]:=\left[\begin{array}{l}
I \\
S
\end{array}\right]\right\} .
$$

The Hamiltonian $H(\boldsymbol{Z})$ in minimal phase space coordinates, which eventually results in the time reparametrized SIR model from (6), is then identified as

$$
H(\boldsymbol{Z}):=\beta[I+S]-\gamma \ln S .
$$

Indeed, the corresponding Hamilton equations deliver a reformulation of the result in (6), i.e.,

$$
\left[\begin{array}{l}
I^{\circ} \\
S^{\circ}
\end{array}\right]=\left[\begin{array}{rr}
0 & 1 \\
-1 & 0
\end{array}\right]\left[\begin{array}{c}
\beta \\
\beta-\gamma / S
\end{array}\right]
$$

Symbolic notation clearly reveals the Hamiltonian structure of the time reparametrized SIR model

$$
\boldsymbol{Z}^{\circ}=\boldsymbol{J} \cdot \partial_{\boldsymbol{Z}} H(\boldsymbol{Z}) .
$$


Thereby, the skew-symmetric $\boldsymbol{J} \in \mathbb{R}^{2} \times \mathbb{R}^{2}$ denotes the so-called symplectic matrix in $\mathbb{P}$

$$
\boldsymbol{J}:=\left[\begin{array}{rr}
0 & 1 \\
-1 & 0
\end{array}\right]
$$

with $\boldsymbol{J}^{t}=-\boldsymbol{J}$ and $\boldsymbol{J}^{2}=-\boldsymbol{I}$, where $\boldsymbol{I} \in \mathbb{R}^{2} \times \mathbb{R}^{2}$ is the common unit matrix in $\mathbb{P}$. The Hamiltonian structure in terms of the skew-symmetric $\boldsymbol{J}$ clearly identifies the (autonomous) Hamiltonian $H(\boldsymbol{Z})$ in minimal phase space coordinates as first integral, i.e., as a conserved quantity under the flow implied by the Hamilton equations, since

$$
H^{\circ}=\partial_{\mathbf{Z}} H \cdot \boldsymbol{Z}^{\circ}=\partial_{\mathbf{Z}} H \cdot \boldsymbol{J} \cdot \partial_{\mathbf{Z}} H=0 .
$$

The gradient of the Hamiltonian $H(\boldsymbol{Z})$ with respect to the minimal phase space coordinates, abbreviated in the sequel as $\boldsymbol{G}(\boldsymbol{Z}) \in \mathbb{R}^{2}$, is computed as

$$
\partial_{\boldsymbol{Z}} H(\boldsymbol{Z})=\left[\begin{array}{c}
\beta \\
\beta-\gamma / S
\end{array}\right]=: \boldsymbol{G}(\boldsymbol{Z}) .
$$

Taken together, the time reparametrized SIR model obeys Hamiltonian structure and identifies the relation between the gradient $\boldsymbol{G}(\boldsymbol{Z}) \in \mathbb{R}^{2}$ of the Hamiltonian $H(\boldsymbol{Z})$ in minimal phase space coordinates and the forcing term $\boldsymbol{F}(\boldsymbol{Z}) \in \mathbb{R}^{2}$ as

$$
Z^{\circ}=\boldsymbol{J} \cdot \boldsymbol{G}(\boldsymbol{Z})=\boldsymbol{F}(\boldsymbol{Z}) .
$$

Exchanging the time derivative to the one with respect to ordinary time $t$ destroys the clean Hamiltonian structure in terms of a constant symplectic matrix; i.e.,

$$
\boldsymbol{Z} \cdot=[S I] \boldsymbol{J} \cdot \boldsymbol{G}(\boldsymbol{Z})=[S I] \boldsymbol{F}(\boldsymbol{Z}) .
$$

One may, of course, reinterpret this result as a Hamiltonian structure with nonconstant, coordinate-dependent symplectic matrix $[S I] J$ on a nonflat manifold.

3.2. Lagrangian in minimal state space coordinate. The minimal state space coordinate, i.e., the generalized coordinate $Q$ defined as the stock of individuals in the infected compartment, spans the one-dimensional state space $\mathbb{S}$; thus,

$$
\mathbb{S}:=\{Q:=I\} .
$$

Then a Legendre transformation of the Hamiltonian in minimal phase space coordinates defines the corresponding Lagrangian

$$
L\left(I, I^{\circ}\right):=\sup _{S}\left\{S I^{\circ}-H(\boldsymbol{Z})\right\} .
$$

The supremum condition identifies $I^{\circ}$ with the derivative $\partial_{S} H(\boldsymbol{Z})$ of the Hamiltonian in minimal phase space coordinates from (9) with respect to the generalized 
momentum, and renders

$$
I^{\circ} \doteq \beta-\gamma / S
$$

Resolving the above supremum condition for $S$ in terms of $I^{\circ}$ delivers

$$
S\left(I^{\circ}\right)=\gamma /\left[\beta-I^{\circ}\right]
$$

Then, with $S\left(I^{\circ}\right) I^{\circ}=\gamma I^{\circ} /\left[\beta-I^{\circ}\right]$ and $H\left(I, S\left(I^{\circ}\right)\right)=\beta\left[I+\gamma /\left[\beta-I^{\circ}\right]\right]-$ $\gamma \ln \left(\gamma /\left[\beta-I^{\circ}\right]\right)$, the Lagrangian in minimal state space coordinate follows eventually as

$$
L\left(I, I^{\circ}\right)=-\beta I-\gamma+\gamma \ln \left(\gamma /\left[\beta-I^{\circ}\right]\right) .
$$

Based on the Lagrangian in minimal state space coordinates, Hamilton's principle results in the stationarity condition

$$
\left[\frac{\partial L}{\partial I^{\circ}}\right]^{\circ}=\frac{\partial L}{\partial I} .
$$

Thus, with $\partial_{I^{\circ}} L=\gamma /\left[\beta-I^{\circ}\right] \rightarrow\left[\partial_{I^{\circ}} L\right]^{\circ}=\gamma I^{\circ \circ} /\left[\beta-I^{\circ}\right]^{2}$ and $\partial_{I} L=-\beta$, the Euler-Lagrange equation corresponding to the Lagrangian in minimal state space coordinates reads

$$
I^{\circ \circ}+R_{0}\left[\beta-I^{\circ}\right]^{2}=0 .
$$

Clearly, the Euler-Lagrange equation in minimal state space coordinates coincides with the single, nonlinear ODE formulation of the time reparametrized SIR model in (7).

3.3. Lagrangian in extended state space coordinates. Alternatively, extended state space coordinates collectively assembled in the column matrix $\boldsymbol{Q} \in \mathbb{R}^{2}$, i.e., the generalized coordinates jointly defined as the stock of individuals in the infected and susceptible compartments, span the two-dimensional state space $\overline{\mathbb{S}}$; thus,

$$
\overline{\mathbb{S}}:=\left\{\boldsymbol{Q}:=\left[\begin{array}{l}
I \\
S
\end{array}\right]\right\}
$$

Then the Lagrangian $\bar{L}\left(\boldsymbol{Q}, \boldsymbol{Q}^{\circ}\right)$ in extended state space coordinates, which eventually results in the time reparametrized SIR model from (6), is determined as

$$
\bar{L}\left(\boldsymbol{Q}, \boldsymbol{Q}^{\circ}\right):=\frac{1}{2} \boldsymbol{Q} \cdot \boldsymbol{J}^{t} \cdot \boldsymbol{Q}^{\circ}-H(\boldsymbol{Q}) .
$$

Here, the Legendre transformation term $\boldsymbol{Q} \cdot \boldsymbol{J}^{t} \cdot \boldsymbol{Q}^{\circ}=\boldsymbol{Q}^{\circ} \cdot \boldsymbol{J} \cdot \boldsymbol{Q}$ expands as the skew-symmetric form

$$
\boldsymbol{Q} \cdot \boldsymbol{J}^{t} \cdot \boldsymbol{Q}^{\circ}=S I^{\circ}-I S^{\circ}
$$


whereas the Hamiltonian $H(\boldsymbol{Q})$, corresponding to (9), is now parametrized in terms of the extended state space coordinates $\boldsymbol{Q}$ with

$$
H(Q):=\beta[I+S]-\gamma \ln S .
$$

As a result, the Lagrangian in extended state space coordinates then renders the stationarity conditions of the corresponding Hamilton principle as

$$
\left[\frac{\partial \bar{L}}{\partial \boldsymbol{Q}^{\circ}}\right]^{\circ}=\frac{\partial \bar{L}}{\partial \boldsymbol{Q}}
$$

With $\partial \boldsymbol{Q}^{\circ} \bar{L}=\boldsymbol{Q} \cdot \boldsymbol{J}^{t} / 2$ and $\partial_{\boldsymbol{Q}} \bar{L}=\boldsymbol{Q}^{\circ} \cdot \boldsymbol{J} / 2-\boldsymbol{G}(\boldsymbol{Q})$, the Euler-Lagrange equations thus follow as

$$
\frac{1}{2} \boldsymbol{Q}^{\circ} \cdot \boldsymbol{J}^{t}=\frac{1}{2} \boldsymbol{Q}^{\circ} \cdot \boldsymbol{J}-\boldsymbol{G}(\boldsymbol{Q}) .
$$

Unfolding the compact symbolic notation, these expand concretely into

$$
\frac{1}{2}\left[\begin{array}{r}
S^{\circ} \\
-I^{\circ}
\end{array}\right]=\frac{1}{2}\left[\begin{array}{r}
-S^{\circ} \\
I^{\circ}
\end{array}\right]-\left[\begin{array}{c}
\beta \\
\beta-\gamma / S
\end{array}\right] .
$$

The Euler-Lagrange equations in (29) are next reformulated by recalling that due to the skew-symmetry of the symplectic matrix, $\boldsymbol{Q}^{\circ} \cdot \boldsymbol{J}=-\boldsymbol{Q}^{\circ} \cdot \boldsymbol{J}^{t}$ and $\boldsymbol{Q}^{\circ} \cdot \boldsymbol{J}^{t}=\boldsymbol{J} \cdot \boldsymbol{Q}^{\circ}$ hold; thus,

$$
\boldsymbol{J} \cdot \boldsymbol{Q}^{\circ}=-\boldsymbol{G}(\boldsymbol{Q})
$$

Finally, with $\boldsymbol{J}^{2}=-\boldsymbol{I}$, the reformulated Euler-Lagrange equations in (31) are modified into

$$
Q^{\circ}=J \cdot G(Q)=F(Q)
$$

Obviously, this format recovers the relation between the gradient $G(\boldsymbol{Q}) \in \mathbb{R}^{2}$ of the Hamiltonian $H(\boldsymbol{Q})$ (in extended state space coordinates) and the forcing term $\boldsymbol{F}(\boldsymbol{Q}) \in \mathbb{R}^{2}$ already established previously in (15). Likewise, exchanging the time derivative to the one with respect to ordinary time $t$

$$
\boldsymbol{Q}^{\bullet}=[S I] \boldsymbol{J} \cdot \boldsymbol{G}(\boldsymbol{Q})=[S I] \boldsymbol{F}(\boldsymbol{Q})
$$

results again in a formulation with nonconstant, coordinate-dependent symplectic matrix $[S I] J$ on a nonflat manifold.

3.4. Hamiltonian in extended phase space coordinates. For academic curiosity it is also interesting to consider extended phase space coordinates $\boldsymbol{Q}$, i.e., the generalized coordinates jointly defined as the stock of individuals in the infected and susceptible compartments, and $\boldsymbol{P}$, i.e., heretofore undefined generalized momenta, 
collectively assembled in the column matrix $\bar{Z} \in \mathbb{R}^{4}$, to span the four-dimensional state space $\overline{\mathbb{P}}$; thus,

$$
\overline{\mathbb{P}}:=\left\{\overline{\boldsymbol{Z}}:=\left[\begin{array}{l}
\boldsymbol{Q} \\
\boldsymbol{P}
\end{array}\right] \text { with } \boldsymbol{Q}:=\left[\begin{array}{l}
I \\
S
\end{array}\right] \text { and } \boldsymbol{P}:=\left[\begin{array}{c}
\Upsilon \\
\Sigma
\end{array}\right]\right\} .
$$

A Legendre transformation of the Lagrangian in extended state space coordinates from (25) defines the associated Hamiltonian

$$
\bar{H}(\boldsymbol{Q}, \boldsymbol{P})=\sup _{\boldsymbol{Q}^{\circ}}\left\{\boldsymbol{P} \cdot \boldsymbol{Q}^{\circ}-\bar{L}\left(\boldsymbol{Q}, \boldsymbol{Q}^{\circ}\right)\right\} .
$$

The corresponding supremum condition identifies $\boldsymbol{P}$ with $\partial \boldsymbol{Q}^{\circ} \bar{L}\left(\boldsymbol{Q}, \boldsymbol{Q}^{\circ}\right)$, the derivative of the Lagrangian $\bar{L}\left(\boldsymbol{Q}, \boldsymbol{Q}^{\circ}\right)$ in extended state space coordinates from (25) with respect to the time reparametrized rate of the generalized coordinates $\boldsymbol{Q}^{\circ}$, and renders

$$
\frac{\partial \bar{L}}{\partial \boldsymbol{Q}^{\circ}}=\frac{1}{2} \boldsymbol{Q} \cdot \boldsymbol{J}^{t}=: \boldsymbol{P} .
$$

As a result, $\boldsymbol{P}$ does not depend on $\boldsymbol{Q}^{\circ}$, thus identifying the Lagrangian $\bar{L}\left(\boldsymbol{Q}, \boldsymbol{Q}^{\circ}\right)$ in (25) as degenerate in the sense of Dirac's generalized Hamiltonian dynamics [Dirac 1950; 1958]. Consequently, with $\boldsymbol{Q} \cdot \boldsymbol{J}^{t}=\boldsymbol{J} \cdot \boldsymbol{Q}$ and $\boldsymbol{J}^{2}=-\boldsymbol{I}$, an additional constraint for the extended phase space coordinates emerges:

$$
\boldsymbol{C}(\boldsymbol{Q}, \boldsymbol{P})=\boldsymbol{Q}+2 \boldsymbol{J} \cdot \boldsymbol{P} \doteq \mathbf{0} .
$$

The Hamiltonian $\bar{H}(\boldsymbol{Q}, \boldsymbol{P})$ in extended phase space coordinates thus follows from Legendre transformation by incorporating the constraint via the Lagrange multiplier $\boldsymbol{\Lambda}$; i.e.,

$$
\bar{H}(\boldsymbol{Q}, \boldsymbol{P})=\frac{1}{2} \boldsymbol{Q} \cdot \boldsymbol{J}^{t} \cdot \boldsymbol{Q}^{\circ}-\bar{L}\left(\boldsymbol{Q}, \boldsymbol{Q}^{\circ}\right)+\boldsymbol{\Lambda} \cdot \boldsymbol{C}(\boldsymbol{Q}, \boldsymbol{P}) .
$$

Taking into account the explicit form of the Lagrangian $\bar{L}\left(\boldsymbol{Q}, \boldsymbol{Q}^{\circ}\right)$ in extended state space coordinates from (25) then renders the explicit representation of the Hamiltonian $\bar{H}(\boldsymbol{Q}, \boldsymbol{P})$ in extended phase space coordinates:

$$
\bar{H}(\boldsymbol{Q}, \boldsymbol{P})=H(\boldsymbol{Q})+\boldsymbol{\Lambda} \cdot \boldsymbol{C}(\boldsymbol{Q}, \boldsymbol{P}) .
$$

Invoking $\partial_{Q} \boldsymbol{C}(\boldsymbol{Q}, \boldsymbol{P})=\boldsymbol{I}$ and $\partial_{\boldsymbol{P}} \boldsymbol{C}(\boldsymbol{Q}, \boldsymbol{P})=2 \boldsymbol{J}$, Hamilton's equations based on the Hamiltonian $\bar{H}(\boldsymbol{Q}, \boldsymbol{P})$ in extended phase space coordinates from (39) result in

$$
\left[\begin{array}{l}
\boldsymbol{Q}^{\circ} \\
\boldsymbol{P}^{\circ}
\end{array}\right]=\left[\begin{array}{rr}
0 & \boldsymbol{I} \\
-\boldsymbol{I} & 0
\end{array}\right]\left[\begin{array}{l}
\partial_{\boldsymbol{Q}} \bar{H} \\
\partial_{\boldsymbol{P}} \bar{H}
\end{array}\right]=\left[\begin{array}{rr}
0 & \boldsymbol{I} \\
-\boldsymbol{I} & 0
\end{array}\right]\left[\begin{array}{c}
\boldsymbol{G}(\boldsymbol{Q})+\boldsymbol{\Lambda} \\
2 \boldsymbol{\Lambda} \cdot \boldsymbol{J}
\end{array}\right] .
$$

The unknown Lagrange multiplier $\boldsymbol{\Lambda}$ is determined from the consistency condition for the constraint

$$
\boldsymbol{C}^{\circ}\left(\boldsymbol{Q}^{\circ}, \boldsymbol{P}^{\circ}\right)=\boldsymbol{Q}^{\circ}+2 \boldsymbol{J} \cdot \boldsymbol{P}^{\circ} \doteq \mathbf{0},
$$


which, upon introducing Hamilton's equations $\boldsymbol{Q}^{\circ}=2 \boldsymbol{\Lambda} \cdot \boldsymbol{J}$ and $\boldsymbol{P}^{\circ}=-[\boldsymbol{G}(\boldsymbol{Q})+\boldsymbol{\Lambda}]$, results in

$$
2 \boldsymbol{\Lambda} \cdot \boldsymbol{J}-2 \boldsymbol{J} \cdot[\boldsymbol{G}(\boldsymbol{Q})+\boldsymbol{\Lambda}] \doteq \mathbf{0} .
$$

The solution of the consistency condition then renders the explicit representation for the Lagrange multiplier

$$
\boldsymbol{\Lambda}=-\frac{1}{2} \boldsymbol{G}(\boldsymbol{Q}) .
$$

Using again Hamilton's equation $\boldsymbol{Q}^{\circ}=2 \boldsymbol{\Lambda} \cdot \boldsymbol{J}$ and exploiting the skew-symmetry of the symplectic matrix, i.e., $\boldsymbol{\Lambda} \cdot \boldsymbol{J}=-\boldsymbol{J} \cdot \boldsymbol{\Lambda}$, recovers once more the already previously established relation between the gradient $\boldsymbol{G}(\boldsymbol{Q}) \in \mathbb{R}^{2}$ of the Hamiltonian $H(\boldsymbol{Q})$ (in extended state space coordinates) and the forcing term $\boldsymbol{F}(\boldsymbol{Q}) \in \mathbb{R}^{2}$ :

$$
Q^{\circ}=\boldsymbol{J} \cdot \boldsymbol{G}(\boldsymbol{Q})=\boldsymbol{F}(\boldsymbol{Q}) \text {. }
$$

Finally, using Hamilton's equation $\boldsymbol{P}^{\circ}=-[\boldsymbol{G}(\boldsymbol{Q})+\boldsymbol{\Lambda}]$ identifies eventually the time reparametrized rate of the heretofore unknown generalized momenta as

$$
\boldsymbol{P}^{\circ}=-\frac{1}{2} \boldsymbol{G}(\boldsymbol{Q})=\frac{1}{2} \boldsymbol{J} \cdot \boldsymbol{F}(\boldsymbol{Q}) .
$$

The last equality follows from $\boldsymbol{J}^{2}=-\boldsymbol{I}$ and the previous relation $\boldsymbol{J} \cdot \boldsymbol{G}(\boldsymbol{Q})=\boldsymbol{F}(\boldsymbol{Q})$.

\section{Coordinate reparametrized SIR model}

Alternatively, and again aiming to recast the basic SIR model into an analytical mechanics format, it is proposed, as a second option, to logarithmically transform the coordinates (or rather the independent variables) as

$$
I \mapsto i:=\ln I \quad \text { and } \quad S \mapsto s:=\ln S .
$$

As a result, the basic SIR model, reparametrized in terms of logarithmically transformed coordinates, however still in terms of derivatives with respect to ordinary time $t$, now reads

$$
\left[\begin{array}{l}
i^{\bullet} \\
s^{\bullet}
\end{array}\right]=f \quad \text { with } \quad f:=\left[\begin{array}{c}
\beta S(s)-\gamma \\
-\beta I(i)
\end{array}\right]=:\left[\begin{array}{l}
v \\
f
\end{array}\right] .
$$

Note that in this reparametrization, the stocks of individuals in the infected and susceptible compartments are considered as dependent functions of the logarithmically transformed coordinates

$$
I(i):=\exp i \text { and } S(s):=\exp s .
$$

In the coordinate reparametrized SIR model the right-hand side is abbreviated as the forcing term $f$, i.e., as the column matrix consisting of the coordinate reparametrized rate of infection $v$ and force of infection $f$. Computing $i^{\bullet}=\beta S(s) s^{\bullet}$ 
from $(47)_{1}$ and eliminating $s^{\bullet}$ with the help of $(47)_{2}$ renders $i^{\bullet \bullet}=-\beta^{2} S(s) I(i)$. Resorting finally again to $(47)_{1}$, i.e., expressing $S(s)=[i \cdot \gamma] / \beta$, allows formulating the coordinate reparametrized SIR model as a single nonlinear ODE

$$
i^{\bullet \bullet}=-\beta I(i)\left[i^{\bullet}+\gamma\right]
$$

exclusively in the logarithmic stock of individuals in the infected compartment.

4.1. Hamiltonian in minimal phase space coordinates. The minimal (logarithmic) phase space coordinates collectively assembled in the column matrix $z \in \mathbb{R}^{2}$, i.e., the generalized coordinate $q$ defined as the logarithmic stock of individuals in the infected compartment and the generalized momentum $p$ defined as the logarithmic stock of individuals in the susceptible compartment, span the two-dimensional phase space $p$; thus,

$$
\mathrm{p}:=\left\{z:=\left[\begin{array}{l}
q \\
p
\end{array}\right]:=\left[\begin{array}{l}
i \\
s
\end{array}\right]\right\}
$$

Next, the Hamiltonian $h(z)$ in minimal (logarithmic) phase space coordinates, which eventually results in the coordinate reparametrized SIR model from (47), is identified as

$$
h(z):=\beta[I(i)+S(s)]-\gamma s .
$$

As a result, the corresponding Hamilton equations deliver a reformulation of (47), i.e.,

$$
\left[\begin{array}{l}
i^{\bullet} \\
s^{\bullet}
\end{array}\right]=\left[\begin{array}{rr}
0 & 1 \\
-1 & 0
\end{array}\right]\left[\begin{array}{c}
\beta I(i) \\
\beta S(s)-\gamma
\end{array}\right]
$$

Symbolic notation showcases clearly the Hamiltonian structure of the (logarithmic) coordinate reparametrized SIR model

$$
\boldsymbol{z}^{\bullet}=\boldsymbol{j} \cdot \partial_{z} h(\boldsymbol{z})
$$

Here, the skew-symmetric $\boldsymbol{j} \in \mathbb{R}^{2} \times \mathbb{R}^{2}$ denotes the appropriate symplectic matrix in $p$

$$
\boldsymbol{j}:=\left[\begin{array}{rr}
0 & 1 \\
-1 & 0
\end{array}\right]
$$

with $\boldsymbol{j}^{t}=-\boldsymbol{j}$ and $\boldsymbol{j}^{2}=-\boldsymbol{i}$, where $\boldsymbol{i} \in \mathbb{R}^{2} \times \mathbb{R}^{2}$ is the common unit matrix in $p$. The Hamiltonian structure in terms of the skew-symmetric $\boldsymbol{j}$ clearly identifies the (autonomous) Hamiltonian $h(z)$ in minimal (logarithmic) phase space coordinates as first integral:

$$
h^{\bullet}=\partial_{z} h \cdot z^{\bullet}=\partial_{z} h \cdot \boldsymbol{j} \cdot \partial_{z} h=0
$$


The gradient of the Hamiltonian $h(z)$ with respect to the minimal (logarithmic) phase space coordinates, abbreviated in the sequel as $g(z) \in \mathbb{R}^{2}$, is computed as

$$
\partial_{z} h(z)=\left[\begin{array}{c}
\beta I(i) \\
\beta S(s)-\gamma
\end{array}\right]=: \boldsymbol{g}(\boldsymbol{z}) .
$$

Summarizing, the coordinate reparametrized SIR model obeys Hamiltonian structure and identifies the relation between the gradient $g(z) \in \mathbb{R}^{2}$ of the Hamiltonian $h(z)$ in minimal (logarithmic) phase space coordinates and the forcing term $\boldsymbol{f}(\boldsymbol{z}) \in \mathbb{R}^{2}$ as

$$
z^{\bullet}=j \cdot g(z)=f(z)
$$

It is emphasized that despite the logarithmic nature of the reparametrized coordinates, here, as a benefit, the Hamiltonian structure involves the constant, coordinateindependent symplectic matrix $\boldsymbol{j}$ of a flat manifold as well as derivatives with respect to ordinary time $t$.

4.2. Lagrangian in minimal state space coordinate. The minimal (logarithmic) state space coordinate, i.e., the generalized coordinate $q$ defined as the logarithmic stock of individuals in the infected compartment, span the one-dimensional state space $s$; thus,

$$
\mathcal{s}:=\{q:=i\}
$$

Legendre transformation of the Hamiltonian in minimal (logarithmic) phase space coordinates defines the corresponding Lagrangian

$$
l\left(i, i^{\bullet}\right)=\sup _{s}\left\{s i^{\bullet}-h(z)\right\} .
$$

Then the supremum condition identifies $i^{\bullet}$ with the derivative $\partial_{s} h(z)$ of the Hamiltonian in minimal (logarithmic) phase space coordinates from (51) with respect to the generalized momentum, and renders

$$
i^{\bullet}=\beta S(s)-\gamma
$$

Resolving the above supremum condition for $s$ in terms of $i^{\bullet}$ delivers

$$
s\left(i^{\bullet}\right)=\ln \left(\left[i^{\bullet}+\gamma\right] / \beta\right) .
$$

Then, with $s\left(i^{\bullet}\right) i^{\bullet}=\ln \left(\left[i^{\bullet}+\gamma\right] / \beta\right) i^{\bullet}$ and $h\left(i, s\left(i^{\bullet}\right)\right)=\beta\left[I(i)+\left[i^{\bullet}+\gamma\right] / \beta\right]-$ $\gamma \ln \left(\left[i^{\bullet}+\gamma\right] / \beta\right)$, the Lagrangian in minimal (logarithmic) state space coordinate follows eventually as

$$
l\left(i, i^{\bullet}\right)=-\beta I(i)-\left[i^{\bullet}+\gamma\right]\left[1-\ln \left(\left[i^{\bullet}+\gamma\right] / \beta\right)\right] .
$$


Based on the Lagrangian in minimal (logarithmic) state space coordinates, Hamilton's principle results in the stationarity condition

$$
\left[\frac{\partial l}{\partial i}\right]^{\bullet}=\frac{\partial l}{\partial i} .
$$

Thus, with $\partial_{i} \bullet l=-\left[1-\ln \left(\left[i^{\bullet}+\gamma\right] / \beta\right)\right]+\beta \rightarrow\left[\partial_{i} \bullet l\right]^{\bullet}=i^{\bullet \bullet} /\left[i^{\bullet}+\gamma\right]$ and $\partial_{i} l=$ $-\beta I(i)$, the Euler-Lagrange equation corresponding to the Lagrangian in minimal (logarithmic) state space coordinates reads

$$
i^{\bullet}+\beta I(i)[i \cdot \gamma]=0 .
$$

Obviously, the Euler-Lagrange equation in minimal (logarithmic) state space coordinates coincides with the single, nonlinear ODE formulation of the coordinate reparametrized SIR model in (49).

4.3. Lagrangian in extended state space coordinates. Alternatively, extended (logarithmic) state space coordinates collectively assembled in the column matrix $\boldsymbol{q} \in$ $\mathbb{R}^{2}$, i.e., the generalized coordinates jointly defined as the logarithmic stock of individuals in the infected and susceptible compartments, span the two-dimensional state space $\overline{\mathrm{s}}$; thus,

$$
\bar{s}:=\left\{\boldsymbol{q}:=\left[\begin{array}{l}
i \\
s
\end{array}\right]\right\} .
$$

The Lagrangian $\bar{l}\left(\boldsymbol{q}, \boldsymbol{q}^{\bullet}\right)$ in extended (logarithmic) state space coordinates, which eventually results in the coordinate reparametrized SIR model from (47), reads

$$
\bar{l}\left(\boldsymbol{q}, \boldsymbol{q}^{\bullet}\right):=\frac{1}{2} \boldsymbol{q} \cdot \boldsymbol{j}^{t} \cdot \boldsymbol{q}^{\bullet}-h(\boldsymbol{q}) .
$$

Here, the Legendre transformation term $\boldsymbol{q} \cdot \boldsymbol{j}^{t} \cdot \boldsymbol{q}^{\bullet}=\boldsymbol{q}^{\bullet} \cdot \boldsymbol{j} \cdot \boldsymbol{q}$ expands as the skewsymmetric form

$$
\boldsymbol{q} \cdot \boldsymbol{j}^{t} \cdot \boldsymbol{q}^{\bullet}=s \boldsymbol{i}^{\bullet}-i s^{\bullet}
$$

The Hamiltonian $h(\boldsymbol{q})$, corresponding to (51), is now parametrized in terms of the extended (logarithmic) state space coordinates $\boldsymbol{q}$ as

$$
h(\boldsymbol{q}):=\beta[I(i)+S(s)]-\gamma s .
$$

In extended (logarithmic) state space coordinates the Lagrangian then renders the stationarity conditions of the corresponding Hamilton principle

$$
\left[\frac{\partial \bar{l}}{\partial \boldsymbol{q}^{\bullet}}\right]^{\bullet}=\frac{\partial \bar{l}}{\partial \boldsymbol{q}}
$$

With $\partial_{\boldsymbol{q}} \cdot \bar{l}=\boldsymbol{q} \cdot \boldsymbol{j}^{t} / 2$ and $\partial_{\boldsymbol{q}} \bar{l}=\boldsymbol{q}^{\bullet} \cdot \boldsymbol{j} / 2-\boldsymbol{g}(\boldsymbol{q})$, the Euler-Lagrange equations thus follow as

$$
\frac{1}{2} \boldsymbol{q}^{\bullet} \cdot \boldsymbol{j}^{t}=\frac{1}{2} \boldsymbol{q}^{\bullet} \cdot \boldsymbol{j}-\boldsymbol{g}(\boldsymbol{q})
$$


and concretely unfold into

$$
\frac{1}{2}\left[\begin{array}{r}
s^{\bullet} \\
-i^{\bullet}
\end{array}\right]=\frac{1}{2}\left[\begin{array}{r}
-s^{\bullet} \\
i^{\bullet}
\end{array}\right]-\left[\begin{array}{c}
\beta I(i) \\
\beta S(s)-\gamma
\end{array}\right] .
$$

Subsequently, the Euler-Lagrange equations in (70) are reformulated by noting that $\boldsymbol{q}^{\bullet} \cdot \boldsymbol{j}=-\boldsymbol{q}^{\bullet} \cdot \boldsymbol{j}^{t}$ and $\boldsymbol{q}^{\bullet} \cdot \boldsymbol{j}^{t}=\boldsymbol{j} \cdot \boldsymbol{q}^{\bullet} ;$ thus,

$$
j \cdot q^{\bullet}=-g(q) .
$$

Finally, with $\boldsymbol{j}^{2}=-\boldsymbol{i}$, the Euler-Lagrange equations from (72) reformulate as

$$
q^{\bullet}=j \cdot g(q)=f(q)
$$

This format recovers the relation between the gradient $\boldsymbol{g}(\boldsymbol{q}) \in \mathbb{R}^{2}$ of the Hamiltonian $h(\boldsymbol{q})$ (in extended (logarithmic) state space coordinates) and the forcing term $\boldsymbol{f}(\boldsymbol{q}) \in \mathbb{R}^{2}$ already established previously in (57).

4.4. Hamiltonian in extended phase space coordinates. For completeness we also consider extended (logarithmic) phase space coordinates $\boldsymbol{q}$, i.e., the generalized coordinates jointly defined as the logarithmic stock of individuals in the infected and susceptible compartments, and $\boldsymbol{p}$, i.e., heretofore undefined generalized momenta, collectively assembled in the column matrix $\bar{z} \in \mathbb{R}^{4}$, to span the four-dimensional state space $\bar{p}$; thus,

$$
\overline{\mathbb{p}}:=\left\{\bar{z}:=\left[\begin{array}{l}
\boldsymbol{q} \\
\boldsymbol{p}
\end{array}\right] \text { with } \boldsymbol{q}:=\left[\begin{array}{l}
i \\
s
\end{array}\right] \text { and } \boldsymbol{p}:=\left[\begin{array}{l}
v \\
\sigma
\end{array}\right]\right\} .
$$

A Legendre transformation of the Lagrangian in extended (logarithmic) state space coordinates from (66) defines the associated Hamiltonian

$$
\bar{h}(\boldsymbol{q}, \boldsymbol{p})=\sup _{\boldsymbol{q}^{\bullet}}\left\{\boldsymbol{p} \cdot \boldsymbol{q}^{\bullet}-\bar{l}\left(\boldsymbol{q}, \boldsymbol{q}^{\bullet}\right)\right\} .
$$

The Legendre transformation identifies $\boldsymbol{p}$ with the derivative $\partial_{\boldsymbol{q}} \cdot \bar{l}\left(\boldsymbol{q}, \boldsymbol{q}^{\bullet}\right)$ of the Lagrangian $\bar{l}\left(\boldsymbol{q}, \boldsymbol{q}^{\bullet}\right)$ in extended (logarithmic) state space coordinates from (66) with respect to the rate of the generalized coordinates $\boldsymbol{q}^{\bullet}$, and renders

$$
\frac{\partial \bar{l}}{\partial \boldsymbol{q}}=\frac{1}{2} \boldsymbol{q} \cdot \boldsymbol{j}^{t}=: \boldsymbol{p}
$$

Thus, $\boldsymbol{p}$ does not depend on $\boldsymbol{q}^{\bullet}$, consequently identifying the Lagrangian $\bar{l}\left(\boldsymbol{q}, \boldsymbol{q}^{\bullet}\right)$ in (66) as degenerate [Dirac 1950; 1958]. Consequently, with $\boldsymbol{q} \cdot \boldsymbol{j}^{t}=\boldsymbol{j} \cdot \boldsymbol{q}$ and $\boldsymbol{j}^{2}=-\boldsymbol{i}$, an additional constraint for the extended (logarithmic) phase space coordinates results:

$$
c(q, p)=q+2 j \cdot p \doteq \mathbf{0}
$$


The Hamiltonian $\bar{h}(\boldsymbol{q}, \boldsymbol{p})$ in extended (logarithmic) phase space coordinates thus follows from Legendre transformation by incorporating the constraint via the Lagrange multiplier $\lambda$; i.e.,

$$
\bar{h}(\boldsymbol{q}, \boldsymbol{p})=\frac{1}{2} \boldsymbol{q} \cdot \boldsymbol{j}^{t} \cdot \boldsymbol{q}^{\bullet}-\bar{l}\left(\boldsymbol{q}, \boldsymbol{q}^{\bullet}\right)+\lambda \cdot \boldsymbol{c}(\boldsymbol{q}, \boldsymbol{p}) .
$$

Taking into account the explicit form of the Lagrangian $\bar{l}\left(\boldsymbol{q}, \boldsymbol{q}^{\bullet}\right)$ in extended (logarithmic) state space coordinates from (66) results in the explicit representation of the Hamiltonian $\bar{h}(\boldsymbol{q}, \boldsymbol{p})$ in extended (logarithmic) phase space coordinates

$$
\bar{h}(\boldsymbol{q}, \boldsymbol{p})=h(\boldsymbol{q})+\lambda \cdot \boldsymbol{c}(\boldsymbol{q}, \boldsymbol{p}) .
$$

Invoking $\partial_{\boldsymbol{q}} \boldsymbol{c}(\boldsymbol{q}, \boldsymbol{p})=\boldsymbol{i}$ and $\partial_{\boldsymbol{p}} \boldsymbol{c}(\boldsymbol{q}, \boldsymbol{p})=2 \boldsymbol{j}$, Hamilton's equations based on the Hamiltonian $\bar{h}(\boldsymbol{q}, \boldsymbol{p})$ in extended (logarithmic) phase space coordinates from (79) result in

$$
\left[\begin{array}{l}
\boldsymbol{q}^{\bullet} \\
\boldsymbol{p}^{\bullet}
\end{array}\right]=\left[\begin{array}{rr}
0 & \boldsymbol{i} \\
-\boldsymbol{i} & 0
\end{array}\right]\left[\begin{array}{l}
\partial_{\boldsymbol{q}} \bar{h} \\
\partial_{\boldsymbol{p}} \bar{h}
\end{array}\right]=\left[\begin{array}{rr}
0 & \boldsymbol{i} \\
-\boldsymbol{i} & 0
\end{array}\right]\left[\begin{array}{c}
\boldsymbol{g}(\boldsymbol{q})+\lambda \\
2 \boldsymbol{\lambda} \cdot \boldsymbol{j}
\end{array}\right]
$$

The unknown Lagrange multiplier $\lambda$ is determined from the consistency condition for the constraint

$$
c^{\bullet}\left(q^{\bullet}, p^{\bullet}\right)=q^{\bullet}+2 j \cdot p^{\bullet}=\mathbf{0}
$$

which, with $\boldsymbol{q}^{\bullet}=2 \lambda \cdot \boldsymbol{j}$ and $\boldsymbol{p}^{\bullet}=-[\boldsymbol{g}(\boldsymbol{q})+\lambda]$, results in

$$
2 \lambda \cdot \boldsymbol{j}-2 \boldsymbol{j} \cdot[\boldsymbol{g}(\boldsymbol{q})+\lambda] \doteq \mathbf{0} .
$$

The solution of the consistency condition then renders the explicit representation for the Lagrange multiplier

$$
\lambda=-\frac{1}{2} g(\boldsymbol{q}) .
$$

Using again Hamilton's equation $\boldsymbol{q}^{\bullet}=2 \boldsymbol{\lambda} \cdot \boldsymbol{j}$ and exploiting $\boldsymbol{\lambda} \cdot \boldsymbol{j}=-\boldsymbol{j} \cdot \boldsymbol{\lambda}$ recovers once more the already previously established relation between the gradient $\boldsymbol{g}(\boldsymbol{q}) \in$ $\mathbb{R}^{2}$ of the Hamiltonian $h(\boldsymbol{q})$ (in extended (logarithmic) state space coordinates) and the forcing term $\boldsymbol{f}(\boldsymbol{q}) \in \mathbb{R}^{2}$ :

$$
q^{\bullet}=j \cdot g(q)=f(q) .
$$

Moreover, using Hamilton's equation $\boldsymbol{p}^{\bullet}=-[\boldsymbol{g}(\boldsymbol{q})+\lambda]$ identifies finally the rate of the heretofore unknown generalized momenta as

$$
p^{\bullet}=-\frac{1}{2} g(q)=\frac{1}{2} \boldsymbol{j} \cdot \boldsymbol{f}(\boldsymbol{q}) .
$$

The last equality follows from $\boldsymbol{j}^{2}=-\boldsymbol{i}$ and the previous relation $\boldsymbol{j} \cdot \boldsymbol{g}(\boldsymbol{q})=\boldsymbol{f}(\boldsymbol{q})$. 


\section{Novel vistas from analytical mechanics}

In order to systematically explore lessons that can be learned from an analytical mechanics viewpoint on mathematical epidemic dynamics modeling, the coordinate reparametrized version of the SIR model, based on the Hamiltonian in phase space, is taken as the point of departure:

$$
z^{\bullet}=j \cdot g(z)
$$

Building on this compact representation, several analytical-mechanics-inspired novel vistas on mathematical epidemic dynamics that promise fruitful research avenues for its modeling are identified in the sequel.

Vista 1. Allowing for nonautonomous, i.e., time-dependent generalized Hamiltonians results in the generalized representation

$$
z^{\bullet}=\boldsymbol{j} \cdot \boldsymbol{g}(z, t) \text {. }
$$

Possible options justifying a nonautonomous Hamiltonian are for example:

(a) Various lockdown measures (cancellation of large events, school closing, contact limitations, etc.) as well as their reversal (exit strategies) at discrete points in time are modeled by time-dependent parameters such as for example the infection and the recovery rates

$$
\beta=\beta(t) \quad \text { and } \quad \gamma=\gamma(t),
$$

thus making the Hamiltonian time-dependent.

(b) Various modifications extend the classical SIR model to account for further compartments such as, e.g., deceased (SIRD model), exposed (SEIR model), and quarantined (SIQRD model), among many other, more sophisticated options [Hethcote 2000; Diekmann et al. 2013]. SIR+ models of these types are then captured by appropriately extending the phase space variables

$$
z:=[I, S, \ldots] \in \mathbb{R}^{2+\cdots}
$$

contributing to the Hamiltonian and its gradient.

(c) Classical SIR-type compartment-based models are coupled ordinary differential equations (ODEs). Extending the ODE-based SIR-type modeling approach to integro-differential equations allows one to also consider, e.g., delay due to incubation time and infectious period [Keimer and Pflug 2020]. For $p$ representing relevant parameters, e.g., continuously distributed risk groups and/or past time, from space $P$, the right-hand side of these read as

$$
\boldsymbol{g}(\boldsymbol{z}, t)=\int_{P} \boldsymbol{\gamma}(z, p, t) \mathrm{d} p
$$

with $\boldsymbol{\gamma}(z, p, t)$ the appropriate $p$-density of $\boldsymbol{g}(z, t)$. 
Vista 2. Allowing for an infinite-dimensional phase space with its coordinates $z=\boldsymbol{z}(\boldsymbol{x}, t) \in \mathbb{R}^{2+\cdots}$ defined as fields in four-dimensional space-time results in the generalized representation

$$
z^{\cdot}=\boldsymbol{j} \cdot \boldsymbol{g}\{\boldsymbol{z}(\boldsymbol{x}), t\} .
$$

Here, the right-hand side is a functional of the phase space coordinates rather than a function. Possible options for an infinite-dimensional phase space are for example:

(a) Gradient-type models, whereby the Hamiltonian depends on the phase space coordinates $\boldsymbol{z}=\boldsymbol{z}(\boldsymbol{x}, t)$ and their higher spatial gradients

$$
\boldsymbol{g}\{\boldsymbol{z}(\boldsymbol{x}), t\}=\delta_{z} h\left(z(\boldsymbol{x}), \nabla_{\boldsymbol{x}} z(\boldsymbol{x}), \ldots, t\right) .
$$

Consequently, the right-hand side follows from the variational derivative of the Hamiltonian, rather than from its gradient. Partial differential equations of reaction-convection-diffusion type describing the spatio-temporal spread of infectious diseases are thus a modeling option [Yamazaki and Wang 2017].

(b) Integral-type models, whereby, similar to peridynamics formulations [Javili et al. 2019], the right-hand side follows from a spatial integration

$$
\boldsymbol{g}\{\boldsymbol{z}(\boldsymbol{x}), t\}=\int_{X} \boldsymbol{\gamma}(z(\boldsymbol{x}), \boldsymbol{x}, t) \mathrm{d} \boldsymbol{x}
$$

over a cut-off domain $X$ (horizon) that covers spatial interaction.

Vista 3. Allowing for a finite-dimensional phase space with its coordinates $\mathbf{z} \in$ $\mathbb{R}^{[2+\cdots] n}$ defined as column matrices results in the generalized representation

$$
\mathbf{z}^{\bullet}=\mathbf{j} \cdot \mathbf{g}(\mathbf{z}, t) .
$$

Possible options for a finite-dimensional phase space are for example:

(a) Partition the entire population into subpopulations, thereby separately considering different age/gender/risk groups (see, e.g., [Pastor-Satorras et al. 2015]):

$$
\mathbf{z}=\left[z_{\text {pop }_{1}}, \ldots, z_{\text {pop }_{\max }}\right] .
$$

(b) Partition into various geographical locations in general network models accounting for the spatio-temporal spread of infectious diseases (see, e.g., [Seroussi et al. 2019]):

$$
\mathbf{z}=\left[z_{\mathrm{loc}_{1}}, \ldots, z_{\mathrm{loc}_{\max }}\right] .
$$

(c) Partition into multiple virus strains providing for generic infectious diseases (see, e.g., [Levy et al. 2018]):

$$
\mathbf{z}=\left[z_{\mathrm{vir}_{1}}, \ldots, \boldsymbol{z}_{\mathrm{vir}_{\max }}\right] .
$$


Capturing the interactions among the various partitions in a network is then reflected by the off-diagonal terms in the Hessian $\mathbf{H}:=\partial_{\mathbf{z z}}^{2} h$ of the Hamiltonian.

Vista 4. For a pandemic such as COVID-19, spatial (geographical) resolution, i.e., resolution of a network, is required at multiple scales: at the global (macro) scale, i.e., for the entire globe; at the medium (meso) scale, i.e., for individual countries; and at the local (micro) scale, i.e., for individual cities/communities. A fully detailed spatial resolution at the local (micro) scale for the entire globe is computationally prohibitive; moreover, most often an overkill degree of detail is also not needed and/or not possible due to the lack of data. However, the spatial resolution shall be adaptive to the quantity of interest, e.g., to study the dynamics of infectious disease spread in a particular city/community, only the integral results of more remote locations on the globe matter. These can be captured by a reduced resolution of the network in those geographically remote locations. This asks for a truly multiscale approach that adaptively zooms in only where needed. Possible options for multiscaling are for example:

(a) Vertical coupling of scales relies on the assumption that the two scales considered are sufficiently separated; see, e.g., [Saeb et al. 2016]. Then the "force" term on the right-hand side can be up-scaled from a subscale model by averaging in the sense of computational homogenization:

$$
\bar{z}^{\bullet}=\langle\mathbf{j} \cdot \mathbf{g}(\mathbf{z}, t ; \bar{z})\rangle .
$$

Here, the sup-scale (indicated by an over-bar) on the left-hand side behaves like an SIR-type model whereas the subscale model at the right-hand side lives either on a finite-dimensional phase space or is represented by a ruledriven, so-called agent-based model. Agent-based models are an alternative modeling paradigm considering only a comparatively small number of individuals (agents). They are capable of capturing the stochastic nature and strong impact of socio-economic factors present at small scales; see, e.g., [German et al. 2020; Rahmandad and Sterman 2008]. The subscale model is driven by the sup-scale phase space coordinates, whereby a proper scale-transition condition defines suited boundary/initial conditions at the subscale.

(b) Horizontal coupling of scales, analogously to the quasicontinuum method [Miller and Tadmor 2002], requires adaptive resolution of the network spacing, here indicated by the sup-script $h$ :

$$
\mathbf{z}_{h}^{\cdot}=\mathbf{j} \cdot \mathbf{g}\left(\mathbf{z}_{h}, t\right) .
$$

Adaptivity requires suited network densification indicators that may follow from a proper error analysis, a topic that is still largely under-investigated for epidemic dynamics models. 
Vista 5. The availability and reliability of recorded data, e.g., regarding the cumulative or daily infection cases, during an epidemic is typically characterized by a large degree of uncertainty, e.g., regarding the infection rates, the degree of immunity, and/or their dark figures. Uncertainty quantification is based on simulations with uncertain data:

$$
\mathbf{z}^{\bullet}=\mathbf{j} \cdot \mathbf{g}(\mathbf{z}(\omega), t)
$$

Thereby, uncertain data is here parametrized in terms of elementary events $\omega$ from which one may repeatedly draw samples to investigate uncertainty propagation throughout our model; see, e.g., [Pivovarov et al. 2018; 2019]. Possible options for the description of uncertainties are for example:

(a) Aleatoric uncertainties require the use of random variables with probability density function (pdf) as a measure of likelihood (e.g., Gaussian pdf in terms of the mean value and standard deviation). Aleatoric uncertainties are stochastic by nature and may not be neglected when the standard deviation is large.

(b) Epistemic uncertainties may be captured by fuzzy variables with possibility density function as a measure of degree of membership (e.g., symmetric triangular membership function in terms of its modal value and support). Epistemic uncertainties reflect a lack of knowledge and, in the case of epidemic dynamics modeling, can be reduced by increasing testing for either infections and/or for antibodies.

Vista 6. The discrete trajectory in time of the phase space variables is algorithmically traced by an integrator of the generic format

$$
\mathbf{z}^{n+1}=\mathbf{z}^{n}+\Delta t \mathbf{j} \cdot \mathbf{g}\left(\mathbf{z}^{n+\alpha}(\omega), t^{n+\alpha}\right) .
$$

Here, subscripts $n+1, n$, and $n+\alpha$ refer to, respectively, the end point, the start point, and an intermediate point of/within a time step of length $\Delta t$. Possible options for time integrators that display different accuracy, stability, and robustness, in particular when integrating nonlinear right-hand sides, are for example:

(a) Runge-Kutta integrators are off-the-shelf algorithms that come in a variety of different flavors (following from the corresponding Butcher tableau) like, e.g., single- and multistage integrators of varying algorithmic accuracy. However, they do not necessarily respect first integrals such as the conservation of the Hamiltonian for autonomous cases and may thus suffer from long-term deterioration of algorithmic stability and robustness. 
(b) Variational integrators are based on a discrete form of the action integral, whereby the integrand of the action integral is given by a discrete Lagrangian

$$
\int_{t^{n}}^{t^{n+1}} l\left(\mathbf{z}^{n+1}, \mathbf{z}^{n}, \Delta t\right) \mathrm{d} t \rightarrow \text { stat. }
$$

The resulting discrete Hamiltonian principle then renders a variational integrator that follows from the discrete action integral being stationary. Variational integrators preserve symmetries (momentum maps) and structure (symplecticity) and are thus characterized by long-term algorithmic accuracy, stability, and robustness; see, e.g., [Lew et al. 2004].

(c) Time-finite-element integrators follow from discretizing the Galerkin (weak) form of the Hamilton equations

$$
\int_{T} \delta \mathbf{z} \cdot\left[\mathbf{z}^{\cdot}-\mathbf{j} \cdot \mathbf{g}\right] \mathrm{d} t=0 \quad \text { for all } \delta \mathbf{z} .
$$

Choosing appropriate Ansatz spaces for the test and trial functions, and suited quadrature rules for approximating the time integrals, render integrators of arbitrary algorithmic accuracy that are also characterized by long-term algorithmic stability and robustness; see, e.g., [Betsch and Steinmann 2000; 2001; 2002; 2005].

Vista 7. The underlying equations governing epidemic dynamics are oftentimes unknown. However, they may be discovered from a data-driven approach [Brunton et al. 2016] if sufficient data is available, a scenario that is typically met for the spatio-temporal spread of infectious diseases. The key idea is then to connect the matrix arrangement of available discrete data points for the rate of the phase space coordinates by a matrix of Ansatz functions, e.g., monomials of the matrix arrangement of available discrete data points for the phase space coordinates, with the matrix arrangement of discrete Ansatz parameters

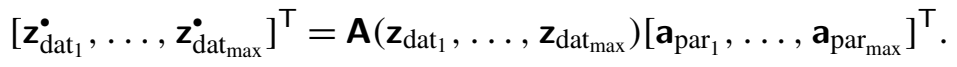

Only few relevant entries in the matrix arrangement of the discrete Ansatz parameters are then determined from sparse regression; consequently the resulting models are denoted as parsimonious and compromise between accuracy and complexity, while avoiding overfitting [Brunton et al. 2016].

Vista 8. Many more modeling approaches inspired by analytical mechanics are conceivable and it is left to the mechanics community to harness those to further improve mathematical epidemic dynamics modeling. 

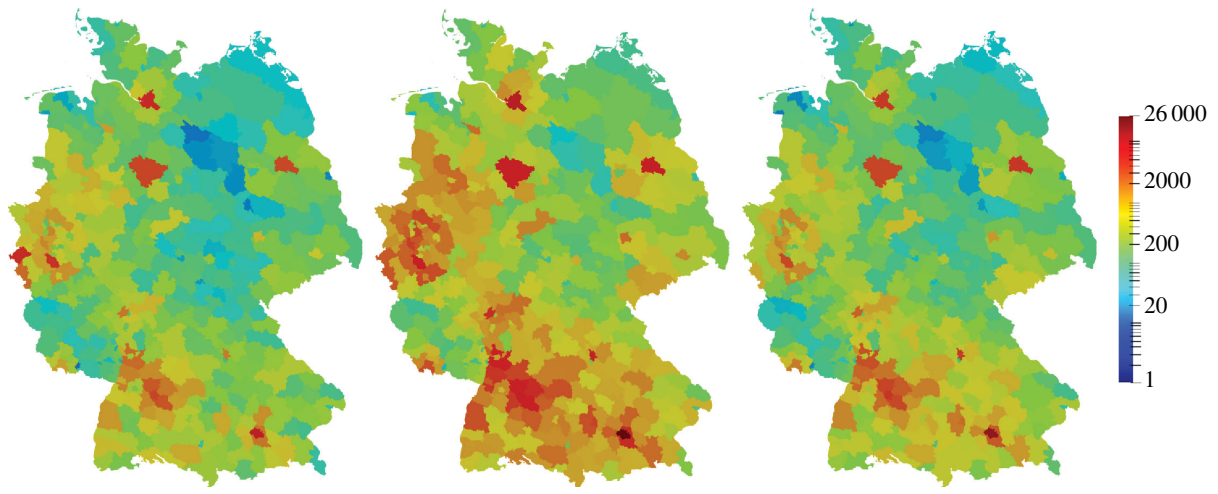

Figure 1. Snapshots of simulated spatio-temporal epidemic spread across Germany with countywise resolution. Colors depict population with COVID-19-specific symptoms on March 13, 2020 (before wave peak), March 27, 2020 (wave peak), and April 10, 2020 (after wave peak). The simulation is based on a spatial network of 401 counties and a convolution-type integro-differential model [Kergaßner et al. 2020a]. The model parameters are calibrated and validated based on extensive data from the Robert Koch Institute (https://corona.rki.de/).

\section{Summary and perspective}

First, this contribution explored options of how to recast the classical SIR model of mathematical epidemic dynamics modeling in the variational setting of analytical mechanics. In particular, it demonstrated that two conceptually entirely different reparametrizations of the basic SIR model, i.e., either by rescaling time or by transforming coordinates (independent variables), severely ease identification of corresponding Hamiltonians and Lagrangians for use within Hamilton's equations and Hamilton's principle. In each case, formulations in either minimal or extended phase and state space coordinates are possible, providing in total eight different modeling options. Interestingly, in minimal phase space coordinates, the stock of individuals in the infected and the susceptible compartments represent the generalized coordinate and the generalized momentum, respectively. In contrast, for extended phase space coordinates, they jointly represent the generalized coordinates, whereas the associated generalized momenta are initially unknown and only follow from exploiting a constraint on the extended phase space coordinates. However, regardless of the particular formulation chosen, from either Hamilton's equations or Hamilton's principle one eventually recovers the original set of coupled ODEs of the SIR model. As a recommendation, logarithmically transforming the coordinates appears more attractive, since derivatives with respect to ordinary time are 
retained for the evolution of the phase space coordinates. As an important perspective, recasting the classical SIR model in one of the eight different modeling options enables the analytical mechanician to employ the full mechanical modeling toolset for a plethora of important extensions. The striking analogy between analytical mechanics and mathematical epidemic dynamics modeling opens up a multitude of fascinating and relevant new research avenues for the progression of the latter. It is thus believed that future exploitation of the Hamiltonian and/or Lagrangian structure of mathematical epidemic dynamics modeling leads to unprecedented insights and options for novel formulations.

As a perspective, Figure 1 showcases (previously unpublished) snapshots of the simulated spatio-temporal epidemic spread across Germany with countywise resolution as an example for the potential of combining, e.g., Vista 1(a)-(c) and Vista 3(b) [Kergaßner et al. 2020a].

\section{Acknowledgement}

I gratefully acknowledge the support of my coauthors for [Kergaßner et al. 2020a; 2020b] in providing the data and simulations for Figure 1.

\section{References}

[Balcan et al. 2009] D. Balcan, V. Colizza, B. Gonçalves, H. Hu, J. J. Ramasco, and A. Vespignani, "Multiscale mobility networks and the spatial spreading of infectious diseases", P. Natl. Acad. Sci. USA 106:51 (2009), 21484-21489.

[Balcan et al. 2010] D. Balcan, B. Gonçalves, H. Hu, J. J. Ramasco, V. Colizza, and A. Vespignani, "Modeling the spatial spread of infectious diseases: the GLobal Epidemic and Mobility computational model", J. Comput. Sci. 1:3 (2010), 132-145.

[Betsch and Steinmann 2000] P. Betsch and P. Steinmann, "Conservation properties of a time FE method, I: Time-stepping schemes for N-body problems”, Int. J. Numer. Meth. Eng. 49:5 (2000), 599-638.

[Betsch and Steinmann 2001] P. Betsch and P. Steinmann, "Conservation properties of a time FE method, II: Time-stepping schemes for non-linear elastodynamics", Int. J. Numer. Meth. Eng. 50:8 (2001), 1931-1955.

[Betsch and Steinmann 2002] P. Betsch and P. Steinmann, "Conservation properties of a time FE method, III: Mechanical systems with holonomic constraints”, Int. J. Numer. Meth. Eng. 53:10 (2002), 2271-2304.

[Betsch and Steinmann 2005] P. Betsch and P. Steinmann, "Conservation properties of a time FE method, IV: Higher order energy and momentum conserving schemes", Int. J. Numer. Meth. Eng. 63:13 (2005), 1849-1897.

[Brunton et al. 2016] S. L. Brunton, J. L. Proctor, and J. N. Kutz, "Discovering governing equations from data by sparse identification of nonlinear dynamical systems", P. Natl. Acad. Sci. USA 113:15 (2016), 3932-3937.

[Diekmann et al. 2013] O. Diekmann, H. Heesterbeek, and T. Britton, Mathematical tools for understanding infectious disease dynamics, Princeton University, 2013. 
[Dirac 1950] P. A. M. Dirac, "Generalized Hamiltonian dynamics", Canadian J. Math. 2 (1950), 129-148.

[Dirac 1958] P. A. M. Dirac, "Generalized Hamiltonian dynamics”, P. Roy. Soc. A Math. Phy. 246:1246 (1958), 326-332.

[German et al. 2020] R. German, A. Djanatliev, L. Maile, P. Bazan, and H. Hackstein, "Modeling exit strategies from COVID-19 lockdown with a focus on antibody tests", preprint, 2020.

[Hethcote 2000] H. W. Hethcote, "The mathematics of infectious diseases", SIAM Rev. 42:4 (2000), 599-653.

[Ionescu et al. 2015] A. Ionescu, R. Militaru, and F. Munteanu, "Geometrical methods and numerical computations for prey-predator systems”, Brit. J. Math. Comp. Sci. 10:5 (2015), 1-15.

[Javili et al. 2019] A. Javili, A. T. McBride, and P. Steinmann, "Continuum-kinematics-inspired peridynamics: mechanical problems”, J. Mech. Phys. Solids 131 (2019), 125-146.

[Keimer and Pflug 2020] A. Keimer and L. Pflug, "Modeling infectious diseases using integrodifferential equations: optimal control strategies for policy decisions and applications in COVID19”, preprint, 2020.

[Kergaßner et al. 2020a] A. Kergaßner, C. Burkhardt, D. Lippold, M. Kergaßner, L. Pflug, D. Budday, P. Steinmann, and S. Budday, "Memory-based meso-scale modeling of Covid-19", Comput Mech. (online publication August 2020).

[Kergaßner et al. 2020b] A. Kergaßner, C. Burkhardt, D. Lippold, S. Nistler, M. Kergaßner, P. Steinmann, D. Budday, and S. Budday, "Meso-scale modeling of COVID-19 spatio-temporal outbreak dynamics in Germany", preprint, 2020.

[Kermack and McKendrick 1927] W. O. Kermack and A. G. McKendrick, "A contribution to the mathematical theory of epidemics”, P. Roy. Soc. A Math. Phy. 115:772 (1927), 700-721.

[Kermack and McKendrick 1932] W. O. Kermack and A. G. McKendrick, "Contributions to the mathematical theory of epidemics, II: The problem of endemicity", P. Roy. Soc. A Math. Phy. 138:834 (1932), 55-83.

[Kermack and McKendrick 1933] W. O. Kermack and A. G. McKendrick, "Contributions to the mathematical theory of epidemics, III: Further studies of the problem of endemicity", P. Roy. Soc. A Math. Phy. 141:843 (1933), 94-122.

[Kermack and McKendrick 1937] W. O. Kermack and A. G. McKendrick, "Contributions to the mathematical theory of epidemics, IV: Analysis of experimental epidemics of the virus disease mouse ectromelia", Epidemiol. Infect. 37:2 (1937), 172-187.

[Kermack and McKendrick 1939] W. O. Kermack and A. G. McKendrick, "Contributions to the mathematical theory of epidemics, V: Analysis of experimental epidemics of mouse-typhoid; a bacterial disease conferring incomplete immunity", Epidemiol. Infect. 39:3 (1939), 271-288.

[Levy et al. 2018] N. Levy, M. Iv, and E. Yom-Tov, "Modeling influenza-like illnesses through composite compartmental models", Phys. A 494 (2018), 288-293.

[Lew et al. 2004] A. Lew, J. E. Marsden, M. Ortiz, and M. West, "Variational time integrators", Internat. J. Numer. Methods Engrg. 60:1 (2004), 153-212.

[Linka et al. 2020] K. Linka, M. Peirlinck, F. Sahli Costabal, and E. Kuhl, "Outbreak dynamics of COVID-19 in Europe and the effect of travel restrictions", Comput. Method. Biomec. 23:11 (2020), $710-717$.

[Lu et al. 2020] H. Lu, C. W. Stratton, and Y.-W. Tang, "Outbreak of pneumonia of unknown etiology in Wuhan, China: the mystery and the miracle", J. Med. Virol. 92:4 (2020), 401-402. 
[Militaru and Munteanu 2013] R. Militaru and F. Munteanu, "Symmetries and conservation laws for biodynamical systems", Int. J. Math. Mod. Meth. Appl. Sci. 7:12 (2013), 965-972.

[Miller and Tadmor 2002] R. E. Miller and E. B. Tadmor, "The Quasicontinuum Method: overview, applications and current directions", J. Comput.-Aided Mater. 9 (2002), 203-239.

[Pastor-Satorras et al. 2015] R. Pastor-Satorras, C. Castellano, P. Van Mieghem, and A. Vespignani, "Epidemic processes in complex networks", Rev. Modern Phys. 87:3 (2015), 925-979.

[Peirlinck et al. 2020] M. Peirlinck, K. Linka, F. Sahli Costabal, and E. Kuhl, "Outbreak dynamics of COVID-19 in China and the United States", Biomech. Model. Mechan. (online publication April 2020).

[Pivovarov et al. 2018] D. Pivovarov, T. Oberleiter, K. Willner, and P. Steinmann, "Fuzzy-stochastic FEM-based homogenization framework for materials with polymorphic uncertainties in the microstructure", Internat. J. Numer. Methods Engrg. 116:9 (2018), 633-660.

[Pivovarov et al. 2019] D. Pivovarov, K. Willner, and P. Steinmann, "On spectral fuzzy-stochastic FEM for problems involving polymorphic geometrical uncertainties", Comput. Methods Appl. Mech. Engrg. 350 (2019), 432-461.

[Rahmandad and Sterman 2008] H. Rahmandad and J. Sterman, "Heterogeneity and network structure in the dynamics of diffusion: comparing agent-based and differential equation models", Manage. Sci. 54:5 (2008), 998-1014.

[Saeb et al. 2016] S. Saeb, P. Steinmann, and A. Javili, "Aspects of computational homogenization at finite deformations: a unifying review from Reuss' to Voigt's bound", Appl. Mech. Rev. 68:5 (2016), art. id. 050801.

[Seroussi et al. 2019] I. Seroussi, N. Levy, D. Paolotti, N. Sochen, and E. Yom-Tov, "On the use of multiple compartment epidemiological models to describe the dynamics of influenza in Europe", 2019. arXiv

[Yamazaki and Wang 2017] K. Yamazaki and X. Wang, "Global stability and uniform persistence of the reaction-convection-diffusion cholera epidemic model", Math. Biosci. Eng. 14:2 (2017), 559579 .

Received 7 Jun 2020. Revised 9 Jul 2020. Accepted 11 Aug 2020.

PAUL STEINMANN: paul.steinmann@fau.de

Institute of Applied Mechanics, Friedrich-Alexander Universität Erlangen-Nürnberg, Erlangen, Germany

and

Glasgow Computational Engineering Centre, University of Glasgow, Glasgow, United Kingdom 



\title{
A GEOMETRICALLY NONLINEAR EULER-BERNOULLI BEAM MODEL WITHIN STRAIN GRADIENT ELASTICITY WITH ISOGEOMETRIC ANALYSIS AND LATTICE STRUCTURE APPLICATIONS
}

\author{
LOC V. TRAN AND JARKKO NIIRANEN
}

\begin{abstract}
The nonlinear governing differential equation and variational formulation of the Euler-Bernoulli beam model are formulated within Mindlin's strain gradient elasticity theory of form II by adopting the von Kármán strain assumption. The formulation can retrieve some simplified beam models of generalized elasticity such as the models of simplified strain gradient theory (SSGT), modified strain gradient theory (MSGT), and modified couple stress theory (MCST). Without the presence of nonlinear terms, the resulting linear differential equation is solvable by analytical means, whereas the mathematical complexity of the nonlinear problem is treated with the Newton-Raphson iteration and a conforming isogeometric Galerkin method with $C^{p-1}$-continuous B-spline basis functions of order $p \geq 3$. Through a set of numerical examples, the accuracy and validity of the present theoretical formulation at linear and nonlinear regimes are confirmed. Finally, an application to lattice frame structures illustrates the benefits of the present beam model in saving computational costs, while maintaining high accuracy as compared to standard $2 \mathrm{D}$ finite element simulations.
\end{abstract}

\section{Introduction}

Microbeams are nowadays the key components in micro- and nanoelectromechanical systems (MEMS and NEMS, respectively) which are broadly applicable in designs such as microsensors and -actuators [Hu et al. 2004; Lun et al. 2006; Moghimi Zand and Ahmadian 2009], atomic force microscopes [Chang et al. 2007; Turner and Wiehn 2001], and so on. In these devices, the beam thickness is sized down to the order of microns and submicrons. A number of experimental tests [Fleck et al. 1994; Lam et al. 2003; Stölken and Evans 1998] have demonstrated, however, that the size-dependent behavior of these extremely small-scale

\section{Communicated by Victor A. Eremeyev.}

This work was supported by the Academy of Finland (grant number 304122).

MSC2010: 65M60, 74A60, 74B20, 74K10, 74Q15.

Keywords: strain gradient elasticity, geometric nonlinearity, beam model, isogeometric analysis, lattice structure. 
microstructural systems cannot be predicted and explained by classical continuum mechanics. In fact, experimental measurements have revealed that reducing the beam/rod size, e.g., decreasing the beam/rod thickness or diameter, results in an enhancement of the torsional stiffness of a copper wire [Fleck et al. 1994], a significant increase in the level of plastic hardening of a thin nickel beam [Stölken and Evans 1998], or a remarkable increase in the bending rigidity of an epoxy beam [Lam et al. 2003]. Interestingly, another class of structures sharing the same nature is microarchitectural structures of any scale [dell'Isola et al. 2016; Khakalo et al. 2018; Khakalo and Niiranen 2019]: size-dependent behavior is an inherent property of materials or metamaterials, present when the characteristic length of the material microstructure becomes comparable with the dimensions of the structure itself, such as the thickness of thin structures. This leads to the necessity of nonclassical continuum theories which include material length scale parameters for predicting size effects, in addition to the classical Lamé constants used in the conventional theory of elasticity.

The nonclassical continuum theories can be classified into two branches: "higherorder" theories proposing additional (internal) variables [Cosserat and Cosserat 1909; Eringen 1999; Green and Rivlin 1964] and "higher-grade" theories including higher gradients of the classical variables, displacements, or strains. In the latter, one of the most well known theories is the strain gradient elasticity theory pioneered by Mindlin [1964; Mindlin and Eshel 1968] and other contemporaries. In the restriction of the present work, we focus only on the strain gradient theory of form II in which the second derivatives of strains are involved. It is worth noting that the three-dimensional isotropic version of Mindlin's theory employs five additional material parameters as compared to the classical isotropic elasticity. Over the last fifty years, many versions of Mindlin's original formulation have been proposed [Lam et al. 2003; Aifantis 1992; Yang et al. 2002] in order to introduce fewer additional material parameters. In the framework of strain gradient elasticity theory, Aifantis's proposal [1992] for a nonlocal version of the generalized Hooke's law introduced only one length scale parameter beside the two conventional Lamé parameters. The corresponding variational formulation was introduced by Altan and Aifantis [1997]. In the framework of this simplified strain gradient theory (SSGT), bending and vibration analysis of beam- and platelike structures, in particular, has been accomplished in [Lazopoulos 2004; 2012; Lazopoulos and Lazopoulos 2010; Askes and Aifantis 2009; Niiranen et al. 2019; 2017; Balobanov and Niiranen 2018]. Lam et al. [2003] simplified Mindlin's formulation to the so-called modified strain gradient theory (MSGT) involving three material length scale parameters. By eliminating two of them, Yang et al. [2002] suggested a modified couple stress theory (MCST) with one additional material parameter again. Based on these two theories, many works involving static and 
dynamic investigations of linear Euler-Bernoulli and Timoshenko beams have been published [Park and Gao 2006; Ma et al. 2008; Kong et al. 2009; Wang et al. 2010]. The reviews in [Lurie and Solyaev 2018; Thai et al. 2017] are suggested for a detailed exposition. Regarding more general models incorporating more nonclassical constitutive parameters but still fewer than in the full anisotropic form of Mindlin's theory, we refer to the following recent contributions: an anisotropic form of the so-called weak nonlocality [Lazar and Po 2015], an anisotropic version of Mindlin's form-II thermoelasticity [Khakalo and Niiranen 2020], a simplified version of Mindlin's second strain gradient (third displacement gradient) elasticity [Khakalo and Niiranen 2018], or microarchitecture-specific second displacement gradient formulations; see, e.g., [Boutin et al. 2017; Rickert et al. 2019; dell'Isola et al. 2019a; 2019b; Abdoul-Anziz and Seppecher 2018].

As seen, the studies in [Lazopoulos 2004; 2012; Lazopoulos and Lazopoulos 2010; Askes and Aifantis 2009; Niiranen et al. 2019; 2017; Balobanov and Niiranen 2018; Park and Gao 2006; Ma et al. 2008; Kong et al. 2009; Wang et al. 2010] are restricted to the linear regime of structural analysis. However, the beam structures used in MEMS or NEMS, or microarchitectural structures, can exhibit large deformations in which the stretching becomes dominant, which results in geometrical nonlinearity which, in turn, results in significant changes in the structural response in both statics and dynamics [Hassanpour et al. 2010; Abdel-Rahman et al. 2002]. Therefore, beside the linear investigations listed above, studies on nonlinearities have gotten attention. For instance, Xia et al. [2010] developed a nonlinear Euler-Bernoulli model based on MCST for the analysis of statics, free vibration, and postbuckling. Asgharis et al. [2010; 2012; Kahrobaiyan et al. 2011] studied the same beam problems by using a nonlinear Timoshenko beam model. Lazopoulos et al. formulated the nonlinear bending and buckling problems of beams [Lazopoulos et al. 2014] and shallow shells [Lazopoulos and Lazopoulos 2011]. In addition, Ramezani [2012; 2013] adopted the multiple scales perturbation technique to solve analytically the geometrically nonlinear beam and plate problems based on strain gradient elasticity. As observed in the aforementioned works, the governing differential equations in the framework of strain gradient theory are mathematically complex due to the appearance of many nonlinear terms involving higher-order derivatives of the variables. Generally, analytical approaches can be utilized only in some simple cases of geometries, loadings, and boundary conditions. Therefore, numerical techniques are necessary. Furthermore, the numerical tools must be somewhat special in cases which require higher-order continuity. Dadgar-Rad and Beheshti [2017] proposed a novel two-node microbeam element based on using fifth-order Hermite functions in order to deal with the stringent continuity requirements. By another way, Hughes et al. [2005] proposed an isogeometric analysis (IGA) utilizing the same basis functions as a B-spline or NURBS in describing the 
geometry to construct the finite approximation. Literature on the computational application of IGA is extremely vast, especially for the beam and plate problems [Bauer et al. 2016; Kiendl et al. 2015; Luu et al. 2015; Tran et al. 2013; Thai et al. 2014; Vo and Nanakorn 2020; Greco and Cuomo 2014; Greco et al. 2017; Greco 2020]. One of the most salient features of the IGA shown clearly in Niiranen's works [Niiranen et al. 2019; 2017] is to use a conforming isogeometric $C^{p-1}$ continuous discretization (with order $p \geq 3$ ) to naturally fulfill the required $C^{2}$ continuity requirement without any additional variables. In the present work, we extend this approach to nonlinear deformations.

First, we formulate a nonlinear strain-gradient-elastic beam model based on Mindlin's strain gradient elasticity theory of form II [Mindlin 1964]. The formulation takes into account the von Kármán strain tensor for geometrical nonlinearity. With proper choices of length scale parameters, we retrieve various one-parameter beam models corresponding to SSGT, MSGT, and MCST and a relation between these models.

Second, we adopt isogeometric B-spline basis functions for implementing a conforming $C^{p-1}$-continuous Galerkin method. Then by applying the NewtonRaphson method, the nonlinear beam bending problem is solved iteratively. Through a set of numerical benchmarks, the accuracy and validity of the present theoretical formulations at linear and nonlinear regimes are confirmed.

Third, we demonstrate the advantages of applying the strain gradient elasticity theory for analyzing 2D triangular lattice structures from the linear regime to the regime of the von Kármán-type geometrical nonlinearity. By using a dimension reduction model, we significantly reduce the number of degrees of freedom, which results in essential savings in computational costs, while maintaining a good level of accuracy, as compared to standard 2D finite element simulations. From the theoretical point of view, it is interesting to witness that beam structures having a triangular, stretching-dominated lattice microarchitecture follow the size-dependent generalized beam models, allowing us to extend the results of [Khakalo et al. 2018; Khakalo and Niiranen 2019] concerning the linear regime of the generalized EulerBernoulli and Timoshenko beam models. From the mechanical point of view, it is crucial that these beam models share the kinematical assumption of straight cross-sectional fibers fulfilled by the lattice beams [Khakalo and Niiranen 2019, Appendix C].

This paper is outlined as follows. The next section details the strain gradient elasticity theory for the Euler-Bernoulli beam model adopting the von Kármán strain assumption. In Section 3, we derive a variational formulation of the beam model for which we then write a conforming Galerkin method based on isogeometric analysis. A set of numerical examples is examined in Section 4. Finally, some concluding remarks close the article. 


\section{Continuum models for generalized beams}

2.1. Strain gradient elasticity theory. To capture the size effects of isotropic materials, Mindlin [1964] proposed the strain energy density of a microstructural solid in a general form with an additional higher-order strain gradient tensor $\xi$ beside the infinitesimal strain tensor $\boldsymbol{\varepsilon}$ appearing in the conventional continuum theories:

$$
\begin{aligned}
U(\boldsymbol{\varepsilon}, \boldsymbol{\xi})=\frac{1}{2} \lambda \varepsilon_{i i} \varepsilon_{j j}+\mu \varepsilon_{i j} \varepsilon_{i j} & \\
& +a_{1} \xi_{i i k} \xi_{k j j}+a_{2} \xi_{i j j} \xi_{i k k}+a_{3} \xi_{i i k} \xi_{j j k}+a_{4} \xi_{i j k} \xi_{i j k}+a_{5} \xi_{i j k} \xi_{k j i}
\end{aligned}
$$

where $\lambda$ and $\mu$ are the classical Lamé constants related to Young's modulus and Poisson's ratio as $\lambda=E v /(1-2 v)(1+v)$ and $\mu=E / 2(1+v)$, whereas the $a_{i}$ $(i=1,2, \ldots, 5)$ are nonclassical material parameters. The components of the thirdorder strain gradient tensor $\xi_{i j k}$ are defined according to the type-II formulation of Mindlin's theory as

$$
\xi_{i j k}=\varepsilon_{j k, i}
$$

where the infinitesimal strain tensor $\varepsilon_{i j}$ is written in terms of the displacement components $u_{i}$ according to the Green strain assumption as usual in continuum mechanics:

$$
\varepsilon_{i j}=\frac{1}{2}\left(u_{i, j}+u_{j, i}+u_{k, i} u_{k, j}\right) .
$$

Note that the symbol $(\cdot),{ }_{i}$ denotes the derivative with respect to coordinate $x_{i}$. The constitutive equations for the Cauchy-like stress and double stress are then given by

$$
\begin{aligned}
& \sigma_{i j}=\frac{\partial U}{\partial \varepsilon_{i j}}=\lambda \varepsilon_{k k} \delta_{i j}+\mu \varepsilon_{i j} \\
& \tau_{i j k}=\frac{\partial U}{\partial \xi_{i j k}}=\frac{1}{2} a_{1}\left(\delta_{i j} \xi_{k p p}\right.\left.+2 \delta_{j k} \xi_{p p i}+\delta_{i k} \xi_{j p p}\right)+2 a_{2} \delta_{j k} \xi_{i p p} \\
&+a_{3}\left(\delta_{i j} \xi_{p p k}+\delta_{i k} \xi_{p p j}\right)+2 a_{4} \xi_{i j k}+a_{5}\left(\xi_{j k i}+\xi_{k i j}\right) .
\end{aligned}
$$

By assigning specific values for the additional material parameters $a_{i}$, certain versions of strain gradient theories can be obtained. For instance, Lam et al. [2003] introduced the modified strain gradient theory with three length scale parameters $l_{i}(i=0,1,2)$ which are used to calculate the five independent parameters $a_{i}$ in Mindlin's form II as

$$
\begin{aligned}
& a_{1}=\mu\left(-\frac{4}{15} l_{1}^{2}+l_{2}^{2}\right), \quad a_{2}=\mu\left(l_{0}^{2}-\frac{1}{15} l_{1}^{2}\right), \\
& a_{3}=\mu\left(-\frac{4}{15} l_{1}^{2}-\frac{1}{2} l_{2}^{2}\right), \quad a_{4}=\mu\left(\frac{1}{3} l_{1}^{2}+l_{2}^{2}\right), \quad a_{5}=\mu\left(\frac{2}{3} l_{1}^{2}-l_{2}^{2}\right) .
\end{aligned}
$$

In the special case of $l_{0}=l_{1}=l_{2}=l$, the nonclassical material parameters $a_{i}$ can be written in terms of one additional length scale parameter as

$$
\left\{a_{1}, a_{2}, a_{3}, a_{4}, a_{5}\right\}=\frac{1}{30} \mu l^{2}\{22,13,-23,40,-10\} .
$$


Similarly, Yang et al. [2002] suggested the modified couple stress theory by eliminating $l_{0}$ and $l_{1}$. As a consequence, (6) is rewritten as

$$
a_{1}=-2 a_{2}=-2 a_{3}=a_{4}=-a_{5}=\mu l^{2} .
$$

In the framework of simplified strain gradient theory [Aifantis 1992], only the nonclassical terms related to $a_{2}$ and $a_{4}$ are considered by introducing a material length scale parameter $g$ as

$$
a_{1}=a_{3}=a_{5}=0, \quad a_{2}=\frac{1}{2} g^{2} \lambda, \quad a_{4}=g^{2} \mu .
$$

2.2. Kinematics of Euler-Bernoulli beams. Let us consider a three-dimensional prismatic beam structure with length $L$, thickness $h$, and width $b$. For simplicity, it is assumed that the beam cross-section $A$ is constant. Within the Euler-Bernoulli hypotheses for in-plane bending, the displacement field of an arbitrary point in the beam is defined as

$$
u_{x}(x, y, z)=u(x)-y w^{\prime}(x), \quad u_{y}(x, y, z)=w(x), \quad u_{z}(x, y, z)=0,
$$

where $u$ and $w$ denote the axial extension and transverse displacement of the beam, respectively. Substituting the displacement field of (10) into (3), with such an assumption that the beam can exhibit large deflection but small or moderate rotation, only one nonzero strain component remains active, expressed according to the von Kármán strain formulation as

$$
\varepsilon_{x x}=u_{x}^{\prime}+\frac{1}{2}\left(u_{y}^{\prime}\right)^{2}=\varepsilon_{0}+y \varepsilon_{1} .
$$

Accordingly, two nonzero components of the strain tensor gradient are obtained according to (2):

$$
\begin{aligned}
& \varepsilon_{x x x}=\varepsilon_{x x, x}=\varepsilon_{2}+y \varepsilon_{3}, \\
& \varepsilon_{y x x}=\varepsilon_{x x, y}=\varepsilon_{1},
\end{aligned}
$$

where these variables can be written in a matrix form as

$$
\hat{\boldsymbol{\varepsilon}}=\left[\begin{array}{c}
\varepsilon_{0} \\
\varepsilon_{1} \\
\varepsilon_{2} \\
\varepsilon_{3}
\end{array}\right]=\left[\begin{array}{c}
u^{\prime}+\frac{1}{2}\left(w^{\prime}\right)^{2} \\
-w^{\prime \prime} \\
u^{\prime \prime}+w^{\prime} w^{\prime \prime} \\
-w^{\prime \prime \prime}
\end{array}\right] .
$$

Herein, the prime stands for a derivative with respect to $x$. By following the constitutive equations in (4) and (5), the nonzero Cauchy-like stress and double stress for the beam are given as

$$
\sigma_{x x}=E \varepsilon_{x x}, \quad \tau_{x x x}=2 \sum_{I=1}^{5} a_{I} \varepsilon_{x x x}=\alpha_{1} \varepsilon_{x x x}, \quad \tau_{y x x}=2\left(a_{2}+a_{4}\right) \varepsilon_{y x x}=\alpha_{2} \varepsilon_{y x x} .
$$




\section{Variational formulation and Galerkin-type isogeometric analysis}

3.1. Variational formulation and boundary conditions. The virtual strain energy in the Euler-Bernoulli beam model based on the strain gradient theory has the form

$$
\begin{aligned}
\delta U & =\int_{V}\left(\sigma_{x x} \delta \varepsilon_{x x}+\tau_{x x x} \delta \varepsilon_{x x x}+\tau_{y x x} \delta \varepsilon_{y x x}\right) d V \\
& =\int_{0}^{L} \int_{A}\left(\sigma_{x x}\left(\delta \varepsilon_{0}+y \delta \varepsilon_{1}\right)+\tau_{x x x}\left(\delta \varepsilon_{2}+y \delta \varepsilon_{3}\right)+\tau_{y x x} \delta \varepsilon_{1}\right) d A d x \\
& =\int_{0}^{L}\left(N \delta \varepsilon_{0}+(M+Q) \delta \varepsilon_{1}+R \delta \varepsilon_{2}+P \delta \varepsilon_{3}\right) d x=\int_{0}^{L} \delta \hat{\boldsymbol{\varepsilon}}^{T} \hat{\boldsymbol{\sigma}} d x,
\end{aligned}
$$

where the classical and nonclassical stress resultants are defined as

$$
\begin{aligned}
& N=\int_{A} \sigma_{x x} d A=E A\left[u^{\prime}+\frac{1}{2}\left(w^{\prime}\right)^{2}\right], \quad M=\int_{A} y \sigma_{x x} d A=-E I w^{\prime \prime}, \\
& R=\int_{A} \tau_{x x x} d A=\alpha_{1} A\left(u^{\prime \prime}+w^{\prime} w^{\prime \prime}\right), \quad P=\int_{A} y \tau_{x x x} d A=-\alpha_{1} I w^{\prime \prime \prime}, \\
& Q=\int_{A} \tau_{y x x} d A=-\alpha_{2} A w^{\prime \prime} .
\end{aligned}
$$

We note that $M$ and $N$ represent the classical stress resultants and $N$ includes a nonlinear strain term as usual, whereas $P, Q$, and $R$ are characteristic for the generalized beam models as follows. $P$ is a parameter-dependent higher-order bending term responsible for possible boundary layers depending on boundary conditions [Niiranen et al. 2019]. $Q$ brings a size dependency to the model, which can be revealed as follows [Niiranen et al. 2019]: when $Q$, proportional to $\alpha_{2} A$, which in turn is proportional to $\alpha_{2} h^{2}$, is combined with $M$, proportional to $E I$, being in turn proportional to $E h^{4}$, one obtains a bending term proportional to $E I\left(1+\alpha_{2} / h^{2}\right)$. The first term of $R$ can be identified as a boundary layer term related to the corresponding bar problem [Niiranen et al. 2016], whereas the second term is a nonclassical term having a link to the nonlinear part of strain. In fact, when the second term of $R$ is combined with $M$ and $Q$, it can be interpreted as a stiffening nonlinear term (actually, equal to $-w^{\prime} Q$ by assuming that $\alpha_{1}=\alpha_{2}$ ).

Equation (16) can be rewritten in a compact form as

$$
\hat{\boldsymbol{\sigma}}=[N, M+Q, R, P]^{T}=\boldsymbol{D} \hat{\boldsymbol{\varepsilon}}
$$

in which $\boldsymbol{D}=\operatorname{diag}\left(E A, E I+\alpha_{2} A, \alpha_{1} A, \alpha_{1} I\right)$ forms a diagonal constitutive matrix.

The virtual work done by the external forces $f_{x}$ and $f_{y}$ can be written as

$$
\delta W=\int_{0}^{L}\left(f_{x} \delta u+f_{y} \delta w\right) d x
$$


and finally the principle of virtual work is expressed in the form

$$
\begin{aligned}
0 & =\delta \Pi=-\delta U+\delta W \\
& =-\int_{0}^{L}\left(N \delta \varepsilon_{0}+(M+Q) \delta \varepsilon_{1}+R \delta \varepsilon_{2}+P \delta \varepsilon_{3}\right) d x+\int_{0}^{L}\left(f_{x} \delta u+f_{y} \delta w\right) d x .
\end{aligned}
$$

Let us next integrate by parts (19) until getting terms which contain the virtual displacements $\delta u$ and $\delta w$ as common factors. Thereafter, the strong form as a pair of governing equations is expressed as

$$
\begin{aligned}
& \text { ( } \delta u) \quad-N^{\prime}+R^{\prime \prime}=f_{x} \text {, } \\
& (\delta w)-\left(N w^{\prime}\right)^{\prime}-(M+Q)^{\prime \prime}+\left(R^{\prime} w^{\prime}\right)^{\prime}+P^{\prime \prime \prime}=f_{y} .
\end{aligned}
$$

Additionally, the corresponding boundary conditions are obtained via the following essential (left) or natural (right) conditions:

$$
\begin{array}{rlrlrl}
u & =\bar{u} & \text { or } & N-R^{\prime} & =\bar{N}, \\
u^{\prime} & =\bar{\alpha} & \text { or } & R & =\bar{R}, \\
w & =\bar{w} & \text { or } & & \left(N-R^{\prime}\right) w^{\prime}+(M+Q)^{\prime}-P^{\prime \prime} & =\bar{V}, \\
w^{\prime} & =\bar{\beta} & \text { or } & -(M+Q)+R w^{\prime}+P^{\prime} & =\bar{M}, \\
w^{\prime \prime} & =\bar{\kappa} & \text { or } & P & =\bar{P} .
\end{array}
$$

The overlined symbols above denote prescribed boundary values, as usual.

By substituting the definitions of the stress resultants in (16) into (20), the governing equation can be rewritten in terms of displacements as

$$
\begin{array}{rl}
-E & A\left[u^{\prime \prime}+w^{\prime} w^{\prime \prime}\right]+\alpha_{1} A\left(u^{(4)}+3 w^{\prime \prime} w^{\prime \prime \prime}+w^{\prime} w^{(4)}\right)=f_{x}, \\
-E A\left[u^{\prime} w^{\prime}+\frac{1}{2} w^{\prime 3}\right]^{\prime} & +\left(E I+\alpha_{2} A\right) w^{(4)} \\
& +\alpha_{1} A\left[\left(u^{\prime \prime \prime} w^{\prime}+w^{\prime 2} w^{\prime \prime \prime}+w^{\prime} w^{\prime \prime 2}\right)\right]^{\prime}-\alpha_{1} I w^{(6)}=f_{y} .
\end{array}
$$

In particular, by eliminating the nonlinear terms in (22) we can obtain the equilibrium equations corresponding to the linear form of the strain gradient EulerBernoulli beam model as a pair of decoupled stretching and bending equations [Niiranen et al. 2019]:

$$
\begin{aligned}
-E A u^{\prime \prime}+\alpha_{1} A u^{(4)} & =f_{x}, \\
\left(E I+\alpha_{2} A\right) w^{(4)}-\alpha_{1} I w^{(6)} & =f_{y}
\end{aligned}
$$


with the respective boundary conditions

$$
\begin{array}{rlrlrl}
u & =\bar{u} & \text { or } & E A u^{\prime}-\alpha_{1} A u^{\prime \prime \prime} & =\bar{N}, \\
u^{\prime} & =\bar{\alpha} & \text { or } & \alpha_{1} A u^{\prime \prime} & =\bar{R}, \\
w & =\bar{w} & \text { or } & & -\left(E I+\alpha_{2} A\right) w^{\prime \prime \prime}+\alpha_{1} I w^{(5)} & =\bar{V}, \\
w^{\prime} & =\bar{\beta} & \text { or } & & \left(E I+\alpha_{2} A\right) w^{\prime \prime}-\alpha_{1} I w^{(4)} & =\bar{M}, \\
w^{\prime \prime} & =\bar{\kappa} & \text { or } & \alpha_{1} I w^{\prime \prime \prime} & =\bar{P} .
\end{array}
$$

Regarding (23) and (24), notice that the axial and transverse displacements are prescribed independently.

3.2. Finite element equations. Solving the nonlinear equation (22) even in the simplest cases is a nontrivial task. Therefore, we prefer solving the problem via a weak form equation based on the discrete formulation of (19) by using the isogeometric finite element method [Hughes et al. 2005]. With an open knot vector $\boldsymbol{\Xi}=\left\{\varsigma_{1}, \varsigma_{2}, \ldots, \varsigma_{m+p+1}\right\}$, which is a nondecreasing sequence of parameter values $\varsigma_{i} \in \mathbb{R}^{+}(i=1,2, \ldots, m+p)$ with $m$ denoting the number of basis functions, the univariate B-spline basis functions $\phi_{i}^{p}(\varsigma)$ are defined recursively by using the Coxde Boor algorithm [Piegl and Tiller 1997]:

$$
\begin{aligned}
\phi_{i}^{p}(\varsigma) & =\frac{\varsigma-\varsigma_{i}}{\varsigma_{i+p}-\varsigma_{i}} \phi_{i}^{p-1}(\varsigma)+\frac{\varsigma_{i+p+1}-\varsigma}{\varsigma_{i+p+1}-\varsigma_{i+1}} \phi_{i+1}^{p-1}(\varsigma) \quad \text { if } p \geq 1, \\
\phi_{i}^{0}(\varsigma) & = \begin{cases}1 & \text { if } \varsigma_{i}<\varsigma<\varsigma_{i+1}, \\
0 & \text { otherwise. }\end{cases}
\end{aligned}
$$

Similar to the traditional finite element method, isogeometric analysis invokes the isoparametric concept in which the displacements are approximated by a linear combination of the basis functions and the unknown degrees of freedom in the form

$$
\boldsymbol{u}^{h}=\sum_{I=1} \phi_{I}(\varsigma) d_{I}
$$

where $\boldsymbol{d}_{I}=\left[u_{I}, w_{I}\right]^{T}$ denotes the degrees of freedom associated to control point $I$.

According to the approximate displacement in (26), the variation of the strain vectors denoted by $\delta \hat{\boldsymbol{\varepsilon}}$ can be computed as

$$
\delta \hat{\boldsymbol{\varepsilon}}=\sum_{I} \boldsymbol{B}_{I} \delta \boldsymbol{d}_{I}
$$

where the generalized strain matrix is defined as

$$
\boldsymbol{B}_{I}=\left[\begin{array}{cccc}
\phi_{I}^{\prime} & 0 & \phi^{\prime \prime} & 0 \\
w^{\prime} \phi_{I}^{\prime} & -\phi_{I}^{\prime \prime} & w^{\prime} \phi_{I}^{\prime \prime}+w^{\prime \prime} \phi_{I}^{\prime} & -\phi_{I}^{\prime \prime \prime}
\end{array}\right]^{T} .
$$


As seen, the third derivative of the basic function is required in (28). Therefore, at least cubic B-spline basis functions $\left(p \geq 3\right.$ ) providing $C^{2}$-continuity are adopted for spanning the approximation space.

By substituting (27) into (19), one can obtain the nonlinear equilibrium equations (after eliminating the arbitrary nodal virtual displacement $\delta \boldsymbol{d}_{I}$ ) as

$$
\boldsymbol{R}(\boldsymbol{d})=\sum_{I} \int_{0}^{L} \boldsymbol{B}_{I}^{T} \hat{\boldsymbol{\sigma}} d x-\boldsymbol{F}_{I}=0
$$

where $\boldsymbol{F}_{I}$ is the load vector

$$
\boldsymbol{F}_{I}=\int_{0}^{L} \phi_{I}\left[f_{x}, f_{y}\right]^{T} d x
$$

The nonlinear equation (29) is solved iteratively by the Newton-Raphson scheme in which the obtained solution is updated by an incremental displacement $\Delta \boldsymbol{d}$ given through the following system of a linear algebraic equation:

$$
\boldsymbol{K}_{T} \Delta \boldsymbol{d}=-\boldsymbol{R}
$$

where the tangent stiffness matrix is defined as

$$
\begin{aligned}
\boldsymbol{K}_{T} & =\frac{\partial \boldsymbol{R}}{\partial \boldsymbol{d}}=\sum_{I} \int_{0}^{L}\left(\boldsymbol{B}_{I}^{T} \frac{\partial \hat{\boldsymbol{\sigma}}}{\partial \boldsymbol{d}}+\frac{\partial \boldsymbol{B}_{I}^{T}}{\partial \boldsymbol{d}} \hat{\boldsymbol{\sigma}}\right) d x \\
& =\sum_{I} \sum_{J} \int_{0}^{L}\left(\boldsymbol{B}_{I}^{T} \boldsymbol{D} \boldsymbol{B}_{J}+N \phi_{I}^{\prime} \phi_{J}^{\prime} \boldsymbol{I}+R\left(\phi_{I}^{\prime} \phi_{J}^{\prime \prime}+\phi_{I}^{\prime \prime} \phi_{J}^{\prime}\right) \boldsymbol{I}\right) d x
\end{aligned}
$$

where $\boldsymbol{I}$ denotes an identity matrix. The iteration is repeated until the difference between two consecutive iterations reduces below a desired error tolerance, e.g., $0.1 \%$. For a detailed description of the solution procedure, one can refer to [Tran et al. 2015; Tran and Kim 2018].

\section{Numerical examples}

4.1. Model comparison. Let us consider a microbeam with thickness $h$, length $L$, and width $b=2 h$ subjected to a concentrated load $Q=100 \mu \mathrm{N}$ placed at the mid-span in the case of simply supported (SS) or clamped (CC) constraints at both ends or at the free-end of a cantilever beam (CF). At first, the assumption of small deformations is adopted for studying the linear behavior of the beams. It is assumed that the beam is made of epoxy with material properties as Young's modulus and Poisson ratio $E=1.44 \mathrm{GPa}$ and $v=0.38$, respectively, and with the length scale parameter assigned to be equal to $l=17.6 \mu \mathrm{m}$ [Lam et al. 2003]. Note that in the case of MSGT, the number of length scale parameters is reduced to one by setting $l_{0}=l_{1}=l_{2}=l=17.6 \mu \mathrm{m}$. The beam dimension is scaled up, by which the ratio 


\begin{tabular}{|r|rrcrccccc|}
\hline & \multicolumn{3}{|c}{ MSGT } & \multicolumn{2}{c}{ MCST } & \multicolumn{2}{c|}{ SSGT } & C \\
$h / l$ & FEM & $l$ & exact & $l$ & $l=33.08$ & $l$ & $l=19.91$ & exact \\
\hline SS 1 & 1.2718 & 1.2722 & 1.2722 & 3.8957 & 1.2733 & 1.601 & 1.270 & 20.8336 \\
5 & 12.8917 & 12.8998 & 12.8997 & 17.7471 & 12.9041 & 14.0603 & 12.8987 & 20.8336 \\
100 & 20.8013 & 20.8016 & 20.8013 & 20.8245 & 20.8016 & 20.8085 & 20.8016 & 20.8336 \\
\hline CC 1 & 0.3173 & 0.3173 & 0.3173 & 0.9739 & 0.3183 & 0.399 & 0.317 & 5.2083 \\
4 & 2.6526 & 2.6526 & 2.6526 & 4.0955 & 2.6571 & 2.9706 & 2.6515 & 5.2083 \\
8 & 4.1973 & 4.1973 & 4.1973 & 4.8771 & 4.2002 & 4.3829 & 4.1966 & 5.2083 \\
100 & 5.1999 & 5.2004 & 5.2003 & 5.2061 & 5.2004 & 5.2021 & 5.2004 & 5.2083 \\
\hline CF 1 & & 20.3678 & 20.3659 & 62.3306 & 20.372 & 25.6345 & 20.3667 & 333.333 \\
4 & & 170.0372 & 170.0373 & 262.1083 & 170.0555 & 190.4536 & 170.0324 & 333.333 \\
8 & & 268.7979 & 268.798 & 312.1289 & 268.8093 & 280.6895 & 268.7949 & 333.333 \\
100 & & 332.8219 & 332.8219 & 333.1885 & 332.822 & 332.9337 & 332.8219 & 333.333 \\
\hline
\end{tabular}

Table 1. Normalized central deflection $10^{3} \widehat{w}$ of the microbeams in the linear regime of deformation $(L / h=30, Q=100 \mu \mathrm{N}, E=$ $1.44 \mathrm{GPa}, v=0.38, l=17.6 \mu \mathrm{m}$, and $b=2 h$ ). See [Dadgar-Rad and Beheshti 2017] for the FEM column and [Timoshenko and Goodier 1970] for the C column.

of the thickness to the length scale parameter $h / l$ changes in the range of $[1,100]$, while the slenderness ratio is kept unchanged at the value $L / h=30$. The maximum normalized deflection $\widehat{w}=w E I /\left(Q L^{3}\right)$ for the three different types of beams based on MSGT, MCST, SSGT, and classical elasticity (C) has been reported in Table 1. For the sake of comparison, the results of Dadgar-Rad's work [Dadgar-Rad and Beheshti 2017] based on MSGT are inserted in the table. In addition, the maximum deflection values based on the classical Euler-Bernoulli beam theory [Timoshenko and Goodier 1970] given as $\widehat{w}=\frac{1}{48}, \frac{1}{192}, \frac{1}{3}$ for the simply supported, clamped, and cantilever beams, respectively, are also supplied. As can be seen, the deflections of classical elasticity are constant and independent of ratios $L / h$ and $h / l$. On the other hand, all nonclassical theories propose lower deflection values which are strongly dependent on ratio $h / l$. It is observed that the discrepancy as compared to the classical elasticity becomes very small as thickness $h$ is far greater than the value of the material length scale parameter $l$, e.g., $l / h=100$. Importantly, the present numerical simulation using IGA and based on only two unknowns ( $u$ and $w)$ produces results in accordance with the FEM results by Dadgar-Rad [Dadgar-Rad and Beheshti 2017] utilizing a nonconforming element with five degrees of freedom per node and also analytical solutions (given in the Appendix) for all of the case studies. For instance, as seen in Figure 1, the numerical results depicted with markers perfectly match the analytical curves plotted with lines. Moreover, 


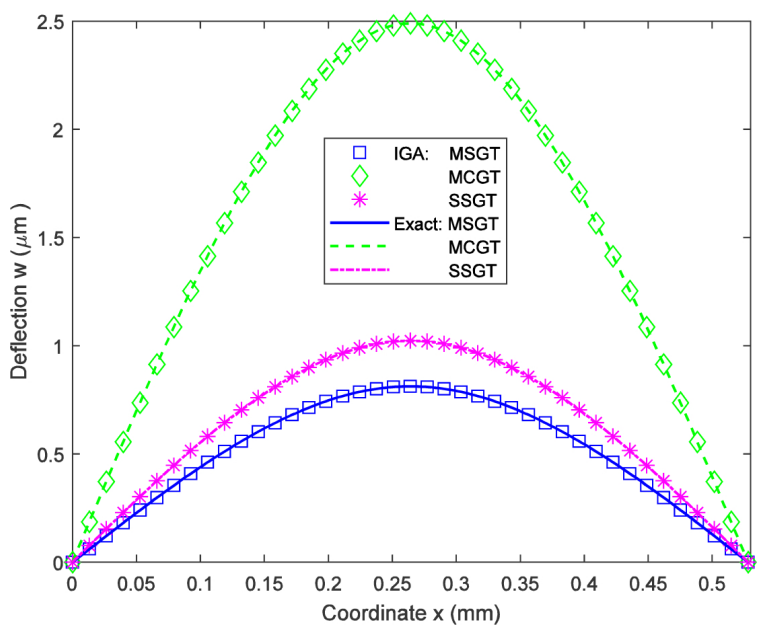

Figure 1. Comparison of various size-dependent theories for a simply supported beam $(L / h=30$ and $h=17.6 \mu \mathrm{m})$ with a concentrated load $Q=100 \mu \mathrm{N}$ at the mid-span. Note that the closed-form results are plotted with lines, while markers depict the numerical results based on IGA.

it is also clearly indicated that different strain gradient elasticity theories produce different results. For example, MCST in the green line, eliminating the sixth-order term in the governing equation (23), overestimates the transverse displacement as compared to the others. To find the relation between these nonclassical models, let us revisit the analytical deflection function in (35) with the values of integration constants given in (37), (39), and (41) according to the different types of boundary constraints. By keeping the integration constant $c_{3}$ fixed, a relation of the material length scale parameter for a particular model is given as

$$
\left(l^{\mathrm{MCST}}, l^{\mathrm{SSGT}}\right)=\left(\sqrt{\frac{53}{15}}, \sqrt{\frac{53}{30(1+v)}}\right) l^{\mathrm{MSGT}} .
$$

According to relation (33), the material length scale parameters of MCST and SSGT are given by factors 33.08 and 19.91, respectively, in accordance with the parameter 17.6 of MSGT. With these factors, the results of the models become practically identical as seen in Table 1 [Niiranen et al. 2019].

As the next step, we again investigate the singly simply supported microbeam with the above data except for a couple of changes: the slenderness ratio is $L / h=$ 20 and the concentrated load is $Q=12 \mathrm{mN}$, in order to make sure that the beam exhibits a relatively large deflection involving geometric nonlinearity. Table 2 lists a tabular comparison between the nonlinear finite element analysis by Dadgar-Rad [Dadgar-Rad and Beheshti 2017] based on the MSGT and classical elasticity and 


\begin{tabular}{|r|rrrrrrrrr|}
\hline & \multicolumn{2}{|c}{ MSGT } & \multicolumn{2}{c}{ MCST } & \multicolumn{2}{c|}{ SSGT } & \multicolumn{2}{c|}{ classical } \\
$h / l$ & \multicolumn{1}{|c}{ FEM } & $l$ & $l$ & $l=33.08$ & $l$ & $l=19.91$ & \multicolumn{1}{c|}{ FEM } & \multicolumn{1}{c|}{ IGA } \\
\hline 1 & 0.9895 & 0.9897 & 1.4253 & 0.9909 & 1.1065 & 0.9894 & 1.7312 & 1.6765 \\
2 & 2.8646 & 2.8649 & 3.6020 & 2.8675 & 3.0727 & 2.8643 & 4.0287 & 3.9591 \\
4 & 7.1612 & 7.1622 & 8.2773 & 7.1663 & 7.4860 & 7.1611 & 8.8529 & 8.7573 \\
100 & 20.8010 & 20.8058 & 20.8287 & 20.8058 & 20.8124 & 20.8054 & 21.0016 & 20.8331 \\
\hline
\end{tabular}

Table 2. Normalized central deflection $10^{3} \widehat{w}$ of a simply supported microbeams considering geometrically nonlinear effect under a concentrated load at mid-span $Q=12 \mathrm{mN}(L / h=20$, $E=1.44 \mathrm{GPa}, v=0.38, l=17.6 \mu \mathrm{m}, b=2 h)$. See [DadgarRad and Beheshti 2017] for the FEM columns.

the present IGA. In addition, we also provide some results using MCST, SSGT, and classical elasticity as well, for comparison. First, let us note that the normalized deflection reduces with an increase in thickness, in contrast to the constant 20.8336 of the classical model. As seen, the smallest beam $(h=l)$ exhibits the largest deformation with relative deflection $w / h=2.165$. Thus, the geometrically nonlinear effect becomes significant and makes the beam stiffer. Meanwhile, the largest beam $(h=100 l)$ reveals a very small relative deflection $w / h=0.003$, indicating no geometrically nonlinear effects. Therefore, the transverse displacement coincides with that of linear analysis - opposite to the finite element method used by Dadgar-Rad [Dadgar-Rad and Beheshti 2017] which overestimates the linear solution. This indicates that the present nonlinear finite element formulation works very well for both linear and nonlinear bending analysis. Furthermore, the same conclusions regarding linear analysis are drawn here. (1) The obtained results are in good agreement with the result using FEM in [Dadgar-Rad and Beheshti 2017], and (2) the nonclassical theories exhibit higher bending rigidity due to the appearance of gradient terms related to the material length scale parameter $l$. However, the effect is no longer dominant in large-scale structures (e.g., $h / l=100$ ). (3) By choosing the material length scale parameter according to (33), the results are practically identical for all the generalized theories. Therefore, in future studies, we prefer to use MSGT as a representative model in the numerical simulations unless otherwise specified.

\subsection{Nonlinear behavior of generalized beams under a uniform distributed load.}

Let us continue to study the nonlinear behavior of the microbeams with thickness $h=17.6 \mathrm{~mm}$ and slenderness ratio $L / h=20$ subjected to a uniform distributed load with magnitude $q=30 \mathrm{~N} / \mathrm{m}$ for the simply supported (SS) beam or $q=60 \mathrm{~N} / \mathrm{m}$ if the beam is clamped (CC). These loading values are chosen to make sure that the maximum rotation of the cross-section does not exceed $15^{\circ}$ (Figures 3 and 4 ), 

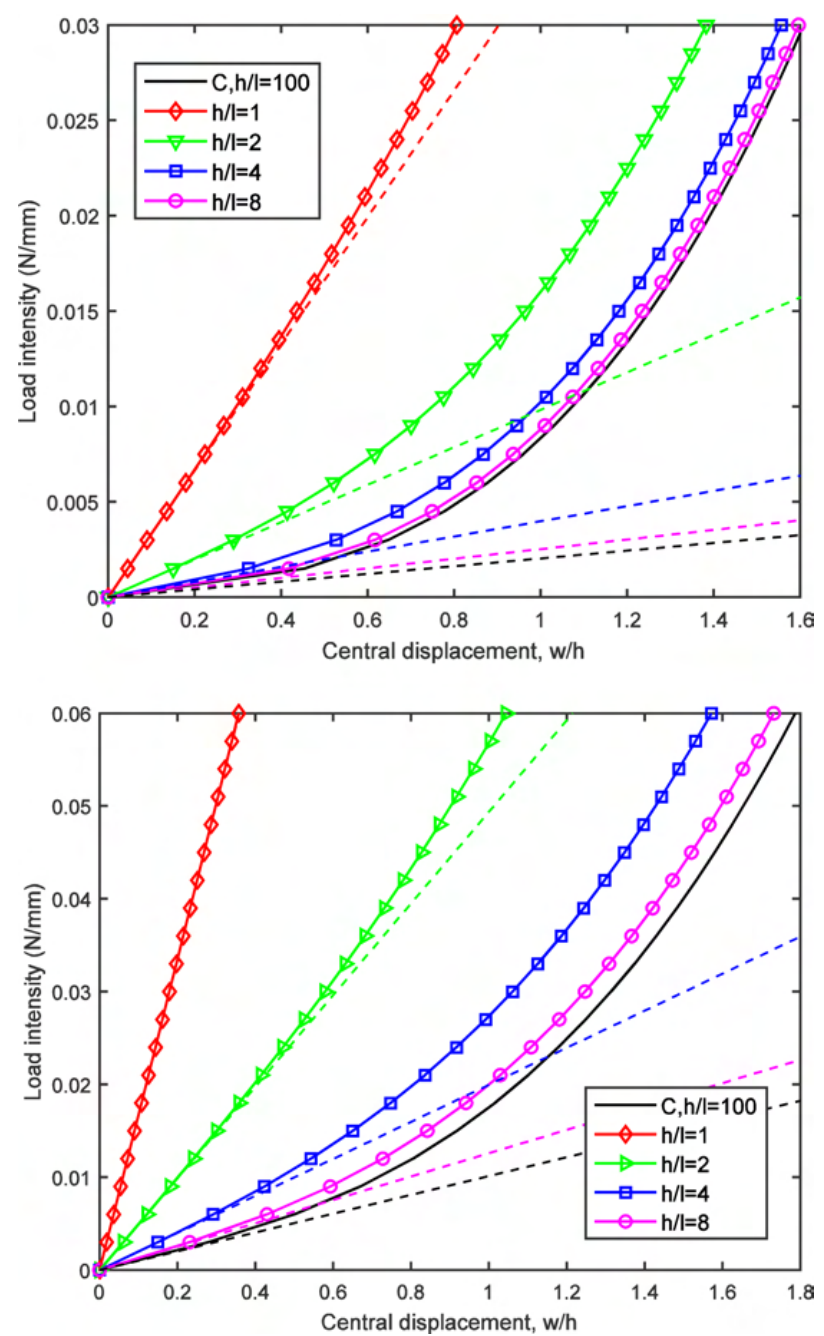

Figure 2. Load displacement curve of a microbeam under different boundary conditions: (bottom) SS and (top) CC (with linear and nonlinear responses plotted with dashed and solid lines, respectively).

which satisfies the moderate rotation limitation of the von Kármán assumption. Figure 2 plots the load displacement curves at the mid-span of the beams for different values of the length scale parameter $l$ scaled down to $[1,2,4,8,100]$ times thickness $h$. It is observed that the linear load displacement responses (dashed lines) are always tangent - at the origin — to the nonlinear load displacement curves (solids lines). Also, the response curves are strongly dependent on ratio $h / l$. As can be seen, the stiffest beam $(h / l=1)$ does not exhibit much nonlinearity, typically 

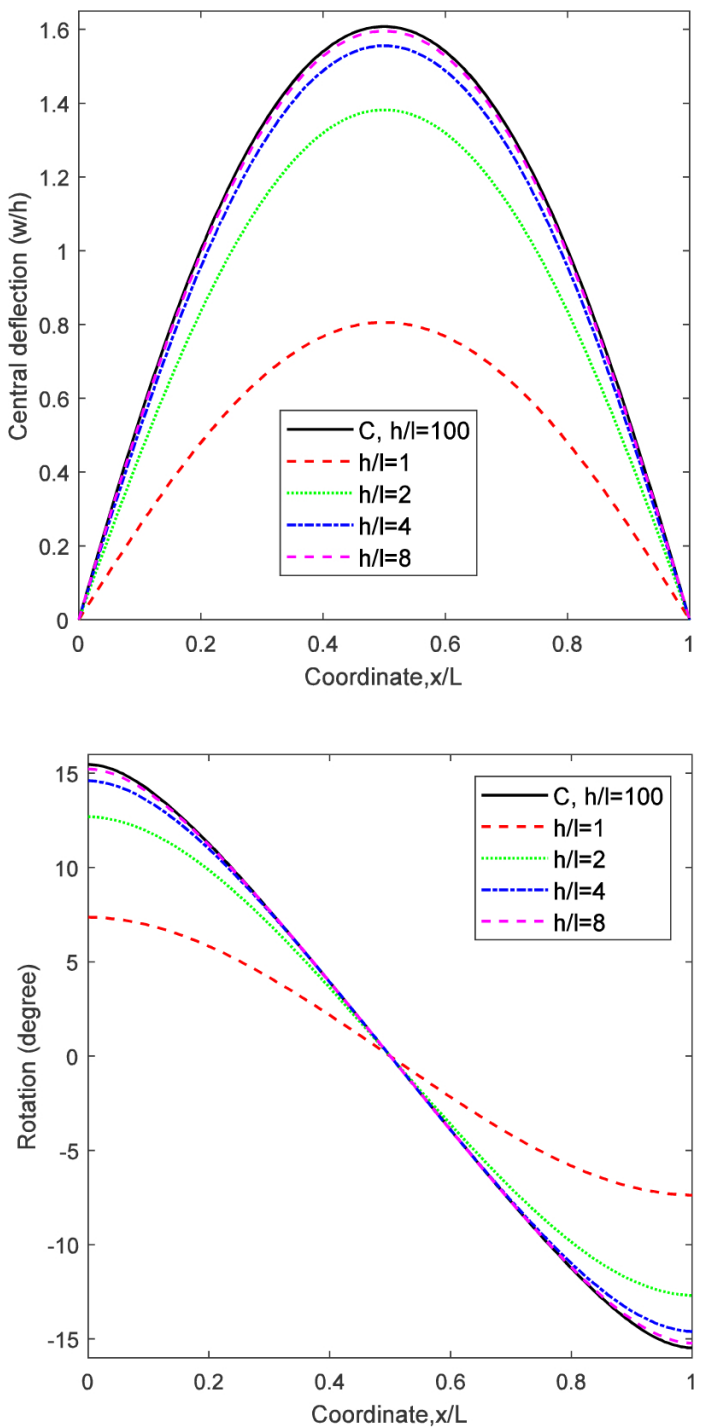

Figure 3. Behavior of a simply supported beam under a uniform load $q=30 \mathrm{~N} / \mathrm{m}$ with $L / h=20$ and $h=17.6 \mu \mathrm{m}$ : (top) the deflection profile and (bottom) the corresponding rotation of the cross-section.

meaning that the linear and nonlinear results coincide (especially for the clamped beam). However, increase in ratio $h / l$ essentially increases the beam deflection. As the deflection-to-thickness ratio becomes high (i.e., $w / h>1$ ), geometric nonlinearity plays a more essential role through the large difference between the linear and nonlinear solutions. To close this subsection, the distributions of transverse 

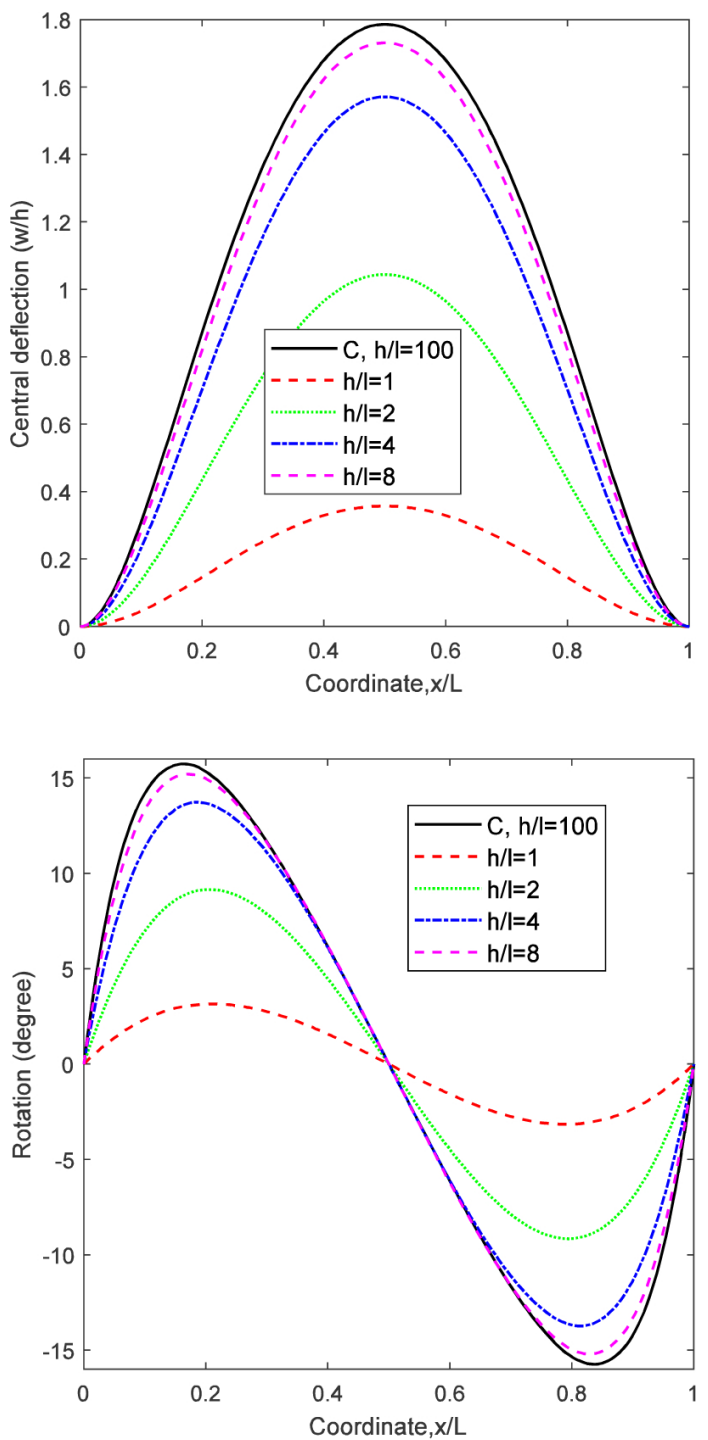

Figure 4. Behavior of a clamped-clamped beam under a uniform load $q=60 \mathrm{~N} / \mathrm{m}$ with $L / h=20$ and $h=17.6 \mu \mathrm{m}$ : (top) the deflection profile and (bottom) the corresponding rotation of the cross-section.

displacement and cross-section rotations through the beam axis coordinate are revealed in Figures 3 and 4. It is noted that the absolute value of rotation based on the strain gradient theory is smaller than that predicted by the classical formulation due to the size effect taken into account. 


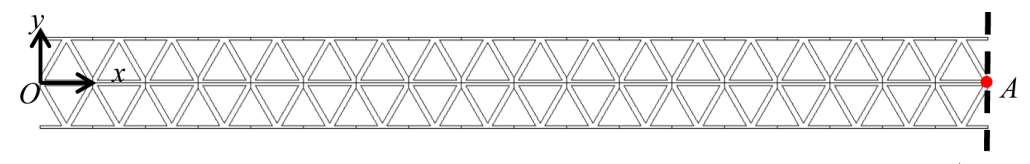

symmetry

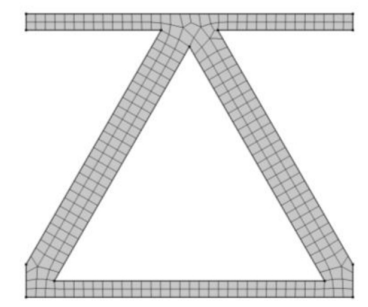

Figure 5. Top: one half of a lattice frame with $L=90 \mathrm{~mm}$ and $h=8.66 \mathrm{~mm}$, produced by replicating a unit cell 18 and 2 times along the $x$-and $y$-axes, respectively. Bottom: 2D finite element mesh of a unit cell in the FEM software COMSOL Multiphysics.

4.3. Application to triangular lattice structures. In order to demonstrate the applicability of strain gradient theories for structures, we further study the linear and geometrically nonlinear behavior of an elastic triangular lattice frame (see Figure 5) with length $L=180 \mathrm{~mm}$ and height $h=8.66 \mathrm{~mm}$. The frame is constrained at two ends by clamped and simply supported conditions and subjected to a uniformly distributed load applied in increments of $\Delta q=4 \mathrm{~N} / \mathrm{m}$ until reaching the final magnitude of $200 \mathrm{~N} / \mathrm{m}$. Due to symmetry, only a half of the frame is modeled as given in Figure 5, top. As seen, the lattice strip can be produced simply by replicating a unit cell or the so-called representative volume element (RVE with the dimensions from [Khakalo et al. 2018, Table 3]). The material properties of the structure are simply Young's modulus $E=2 \mathrm{GPa}$ and Poisson's ratio $v=0.25$.

A reference model of the structure is built by using linear quadrilateral finite elements of classical elasticity in COMSOL Multiphysics with a mesh for each unit cell shown in Figure 5, bottom. The structure can be modeled by a one-dimensional generalized beam model as a homogenized isotropic beam with equivalent mechanical properties as $E_{\text {eff }}=246.7 \mathrm{MPa}$ and $\nu_{\text {eff }}=0.335$ and intrinsic material length scale parameter $l=1.57 \mathrm{~mm}$ [Khakalo et al. 2018].

The maximum deflection of the beam is recorded at the mid-span and plotted for each load increment in Figure 6. The red solid lines denote the nonlinear solutions, while red dashed lines correspond to the linear ones, both corresponding to the 2D reference model. As seen, the present results from the 1D strain gradient beam model with red circles and diamond markers, corresponding to the linear and nonlinear regimes, respectively, are nearly lying on the curves. This means that the strain gradient beam model captures the bending behavior of the lattice 

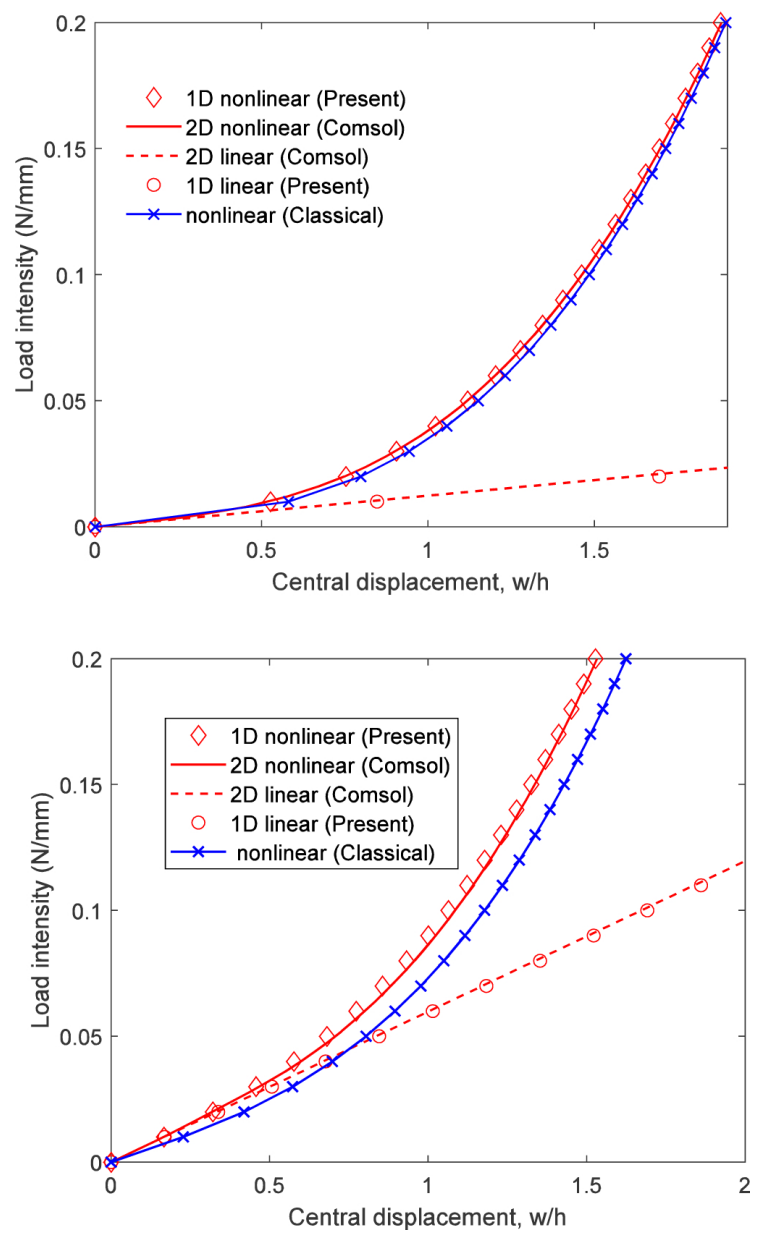

Figure 6. Load displacement curves of a lattice beam under uniform load $q=200 \mathrm{~N} / \mathrm{m}$ with $h=8.66 \mathrm{~mm}, L=180 \mathrm{~mm}$, and $l=1.57 \mathrm{~mm}$ with (top) simply supported and (bottom) clamped boundary conditions.

frame in both linear and nonlinear regimes. Again, the same observation as for Figure 2 is that the predicted deflection of the lattice frame (or the strain gradient beam model) is always smaller than that of the classical beam theory plotted with a blue curve. Furthermore, a comparison of the deflection distribution between the 2D reference and 1D beam simulations is plotted in Figure 7. As observed, the present generalized beam model is in good accordance with the global deflection of the frame represented through the mid-line OA but, naturally, does not describe in detail any local behavior such as bending or buckling of single struts near the clamped end of the frame as shown in Figure 8, bottom. 


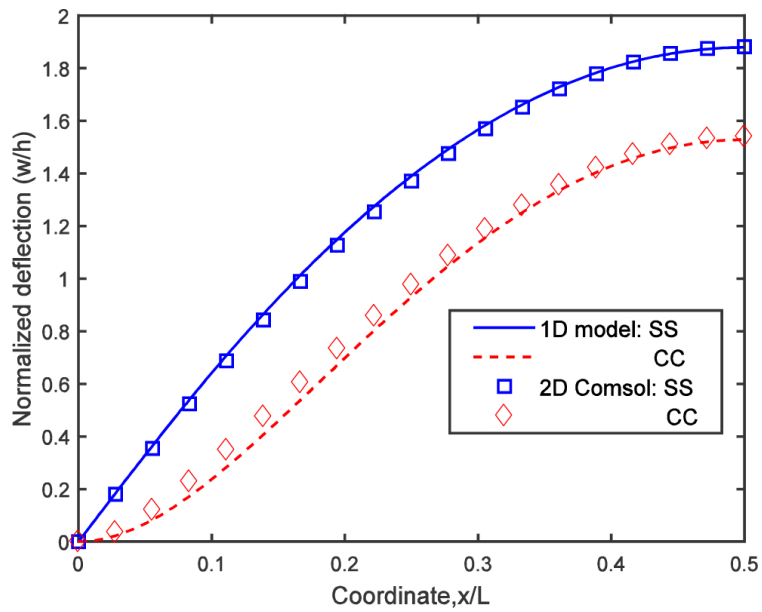

Figure 7. A comparison for the deflection distribution along the mid-line between the $1 \mathrm{D}$ beam model and $2 \mathrm{D}$ reference simulations with clamped (CC) and simply supported (SS) boundary conditions.

\begin{tabular}{|l|rrrr|}
\hline & \multicolumn{2}{|c}{ 2D reference model } & \multicolumn{2}{c|}{ 1D beam model } \\
$N$ & DoFs & time (s) & DoFs & time (s) \\
\hline 2 & 165366 & 258 & & \\
4 & 651562 & 880 & 24 & 0.5 \\
8 & 2586450 & 4416 & & \\
\hline
\end{tabular}

Table 3. Number of DoFs and time consumption for the present 1D beam model and the 2D reference model.

To end this subsection, we discuss the computational efficiency of the present beam model by studying clamped lattice structure strips subjected to a concentrated load $P=30 \mathrm{~N}$ at the mid-span. A series of beam-like lattice structures is formed by scaling up the frame studied above having two $(N=2)$ unit cells in the thickness direction. The subsequent structures of the series have four and eight $(N=4$ and $N=8$ ) unit cells in the thickness direction, whereas the slenderness of the beam-like structures is kept constant: $L / h=20.7$. Table 3 shows the number of degrees of freedom (DoFs) and time consumption in the 2D reference and 1D beam simulations. As seen, in 2D simulations doubling factor $N$ implies an increase of $N^{2}$ DoFs. Meanwhile, the present strain gradient beam model requires 8 elements associated with $24 \mathrm{DoFs}$ (as using quartic B-spline basic functions with $q=4$ ) but still achieves a good agreement with the 2D solutions as shown in Figure 9. (Both programs are compiled on a desktop PC with an Intel Core i7-7600U $2.80 \mathrm{GHz}$ CPU and 16 GB of RAM.) 

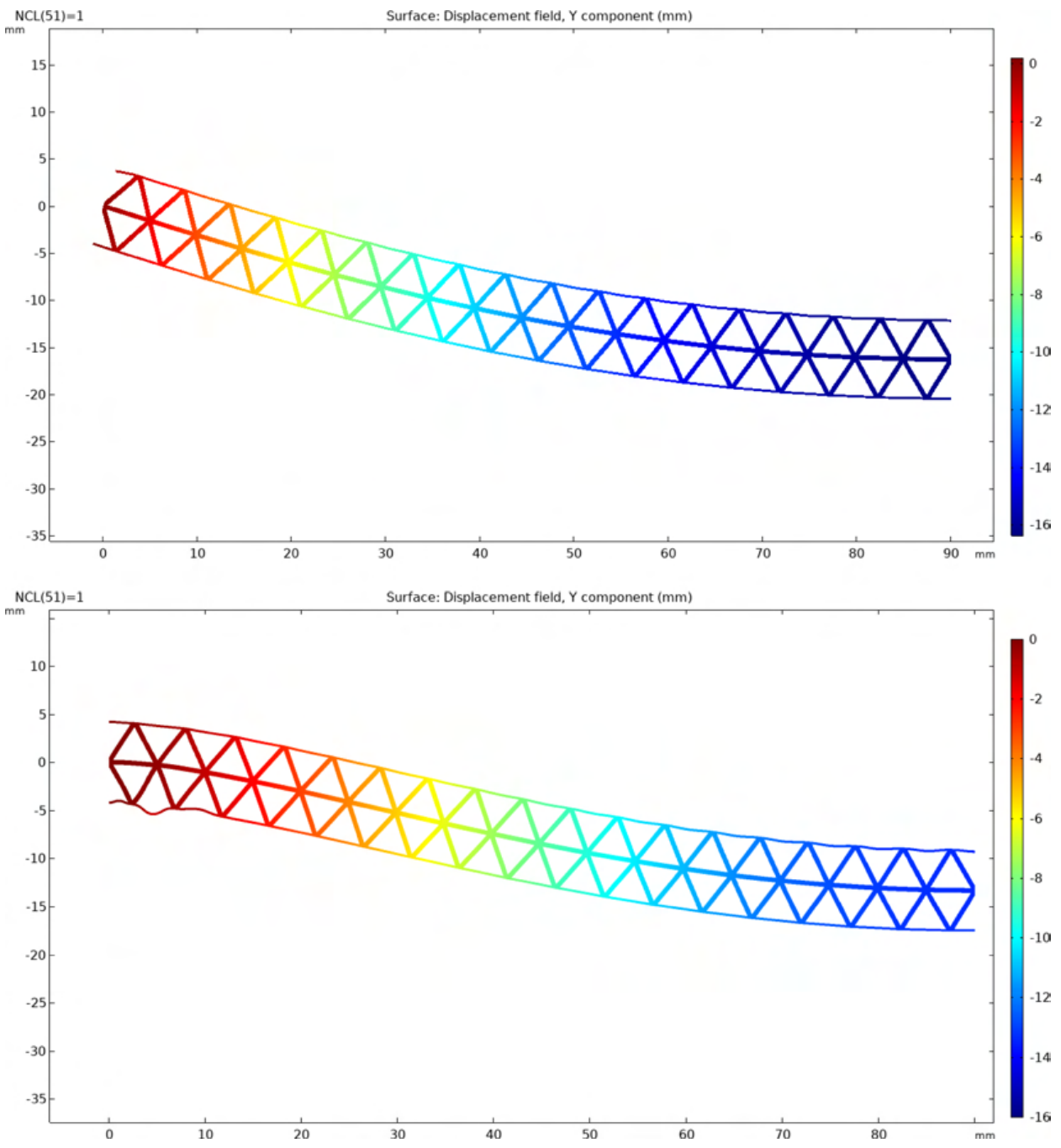

Figure 8. The transverse displacement of a lattice frame modeled by $2 \mathrm{D}$ finite elements (with COMSOL Multiphysics) under simply supported (top) and clamped (bottom) boundary conditions.

\section{Conclusions}

This paper studies the nonlinear bending of the Euler-Bernoulli beam model within Mindlin's strain gradient elasticity theory of form II retrieved with simplified oneparameter beam models of generalized elasticity. In principle, different beam models give different results. In practice, however, by choosing the value of the length scale parameter properly, almost identical results can be obtained - for both linear and nonlinear regimes of deformation. Furthermore, the geometrical nonlinearity 


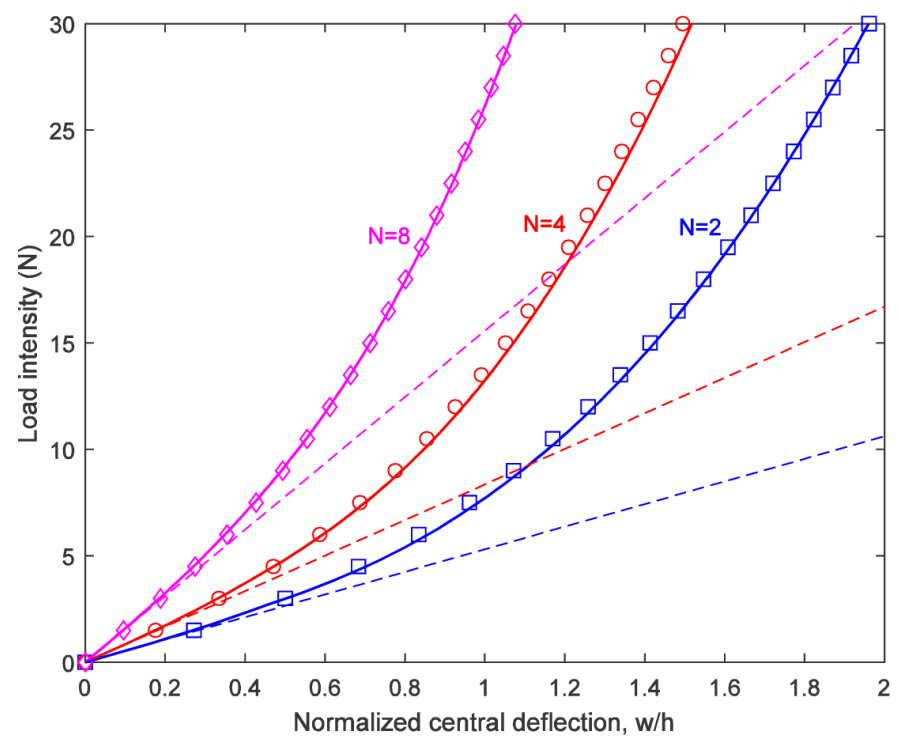

Figure 9. Load displacement curves for structures with $N$ unit cells in the thickness direction: linear solutions (dashed lines), nonlinear solutions with 2D reference model (solid lines), and 1D beam model (markers).

and size effects reduce the deflection of the beams as compared to the classical theory of elasticity, especially as the material length scale parameter becomes comparable to the beam thickness. It is noted that according to the von Kármán strain assumptions nonlinear deformations are limited to small or moderate rotations. More general large deformations are left for further works.

For the computational part, besides analytical results for some benchmark problems, a conforming and isogeometric B-spline Galerkin discretization is adopted for numerical solutions. With basis functions of order $p \geq 3$, the method naturally satisfies the stringent $C^{2}$-continuity required by the strain gradient beam model.

Finally, we demonstrate the advantage of the present beam model by studying 2D lattice frame structures. With a $1 \mathrm{D}$ beam model, we significantly reduce the number of DoFs but still maintain a good level of accuracy as compared with 2D reference simulations. However, the beam model as a homogenized model describing the mid-line of the beam does not describe such behavior as local bending or buckling of single lattice struts in the vicinity of concentrated loads or constrained boundaries. A homogenization method considering initial imperfections for geometrically nonlinear analysis [Reinaldo Goncalves et al. 2016] could be considered to treat this issue in future studies. 


\section{Appendix: Analytical solution for linear statics of strain-gradient-elastic thin beams}

First, let us consider a singly simply supported beam with a concentrated load $Q$ at the mid-span. The governing equation related to the transverse displacement can be derived from (23) by setting $f_{y}=0$, giving

$$
\left(E I+\alpha_{2} A\right) w^{(4)}-\alpha_{1} I w^{(6)}=0 .
$$

The analytical solution of (34) is given by

$$
w(x)=c_{0}+c_{1} x+c_{2} x^{2}+c_{3} x^{3}+c_{4} e^{\beta x}+c_{5} e^{-\beta x}
$$

in which $\beta=\sqrt{\left(E I+\alpha_{2} A\right) / \alpha_{1} I}$ and the six integration constants $c_{I}(I=0,1, \ldots, 5)$ are determined from the essential and natural boundary conditions. In this beam problem, due to symmetry, a half of the beam is considered with boundary conditions

$$
\begin{aligned}
w(0) & =0, & \bar{V}(L / 2) & =Q / 2, \\
\bar{M}(0) & =0, & w^{\prime}(L / 2) & =0, \\
w^{\prime \prime \prime}(0) & =0, & w^{\prime \prime \prime}(L / 2) & =0 .
\end{aligned}
$$

With these constrains, the integration constants are defined as

$$
\begin{array}{ll}
c_{0}=\frac{6 c_{3}}{\beta^{3} \sinh (\beta L / 2)}(1-\cosh (\beta L / 2)), & c_{3}=\frac{-Q}{12\left(E I+\alpha_{2} A\right)}, \\
c_{1}=c_{3}\left(\frac{6}{\beta^{2}}-\frac{3 L^{2}}{4}\right), & c_{4}=\frac{-3 c_{3}}{\beta^{3} \sinh (\beta L / 2)}\left(1-e^{-\beta L / 2}\right), \\
c_{2}=0, & c_{5}=\frac{-3 c_{3}}{\beta^{3} \sinh (\beta L / 2)}\left(1-e^{\beta L / 2}\right) .
\end{array}
$$

Second, analogously, a singly clamped beam with a concentrated load at the mid-span satisfies the boundary conditions

$$
\begin{aligned}
w(0) & =0, & \bar{V}(L / 2) & =Q / 2, \\
w^{\prime}(0) & =0, & w^{\prime}(L / 2) & =0, \\
w^{\prime \prime \prime}(0) & =0, & w^{\prime \prime \prime}(L / 2) & =0 .
\end{aligned}
$$


The integration constants are accordingly defined as

$$
\begin{aligned}
c_{0} & =\frac{6 c_{3}}{\beta^{3} \sinh (\beta L / 2)}(1-\cosh (\beta L / 2)), & c_{3} & =\frac{-Q}{12\left(E I+\alpha_{2} A\right)}, \\
c_{1} & =\frac{6 c_{3}}{\beta^{2}}, & c_{4} & =\frac{-3 c_{3}}{\beta^{3} \sinh (\beta L / 2)}\left(1-e^{-\beta L / 2}\right), \\
c_{2} & =-\frac{3 L c_{3}}{4}, & c_{5} & =\frac{-3 c_{3}}{\beta^{3} \sinh (\beta L / 2)}\left(1-e^{\beta L / 2}\right) .
\end{aligned}
$$

Third, a cantilever beam subjected to loading $Q$ at the free end gets the integration constants of (41) which satisfy the boundary conditions

$$
\begin{aligned}
w(0) & =w^{\prime}(0)=0, \\
w^{\prime \prime \prime}(0) & =w^{\prime \prime \prime}(L)=0, \\
\bar{V}(L) & =Q, \quad \bar{M}(L)=0
\end{aligned}
$$

and integration constants

$$
\begin{aligned}
c_{0} & =\frac{6 c_{3}}{\beta^{3} \sinh (\beta L)}(1-\cosh (\beta L)), & c_{3} & =\frac{-Q}{6\left(E I+\alpha_{2} A\right)}, \\
c_{1} & =6 c_{3} / \beta^{2}, & c_{4} & =\frac{-3 c_{3}}{\beta^{3} \sinh (\beta L)}\left(1-e^{-\beta L}\right), \\
c_{2} & =-3 c_{3} L, & c_{5} & =\frac{-3 c_{3}}{\beta^{3} \sinh (\beta L)}\left(1-e^{\beta L}\right),
\end{aligned}
$$

\section{References}

[Abdel-Rahman et al. 2002] E. M. Abdel-Rahman, M. I. Younis, and A. H. Nayfeh, "Characterization of the mechanical behavior of an electrically actuated microbeam", J. Micromech. Microeng. 12:6 (2002), 759-766.

[Abdoul-Anziz and Seppecher 2018] H. Abdoul-Anziz and P. Seppecher, "Strain gradient and generalized continua obtained by homogenizing frame lattices", Math. Mech. Complex Syst. 6:3 (2018), 213-250.

[Aifantis 1992] E. C. Aifantis, "On the role of gradients in the localization of deformation and fracture”, Internat. J. Engrg. Sci. 30:10 (1992), 1279-1299.

[Altan and Aifantis 1997] B. S. Altan and E. C. Aifantis, "On some aspects in the special theory of gradient elasticity”, J. Mech. Behav. Mat. 8:3 (1997), 231-282.

[Asghari et al. 2010] M. Asghari, M. H. Kahrobaiyan, and M. T. Ahmadian, "A nonlinear Timoshenko beam formulation based on the modified couple stress theory", Internat. J. Engrg. Sci. 48:12 (2010), 1749-1761.

[Asghari et al. 2012] M. Asghari, M. H. Kahrobaiyan, M. Nikfar, and M. T. Ahmadian, "A sizedependent nonlinear Timoshenko microbeam model based on the strain gradient theory", Acta Mech. 223:6 (2012), 1233-1249.

[Askes and Aifantis 2009] H. Askes and E. C. Aifantis, "Gradient elasticity and flexural wave dispersion in carbon nanotubes", Phys. Rev. B 80:19 (2009), art. id. 195412. 
[Balobanov and Niiranen 2018] V. Balobanov and J. Niiranen, "Locking-free variational formulations and isogeometric analysis for the Timoshenko beam models of strain gradient and classical elasticity", Comput. Methods Appl. Mech. Engrg. 339 (2018), 137-159.

[Bauer et al. 2016] A. M. Bauer, M. Breitenberger, B. Philipp, R. Wüchner, and K.-U. Bletzinger, "Nonlinear isogeometric spatial Bernoulli beam", Comput. Methods Appl. Mech. Engrg. 303 (2016), 101-127.

[Boutin et al. 2017] C. Boutin, F. dell'Isola, I. Giorgio, and L. Placidi, "Linear pantographic sheets: asymptotic micro-macro models identification”, Math. Mech. Complex Syst. 5:2 (2017), 127-162.

[Chang et al. 2007] W.-J. Chang, H.-L. Lee, and T. Y.-F. Chen, "Study of the sensitivity of the first four flexural modes of an AFM cantilever with a sidewall probe", Ultramicroscopy 108:7 (2007), 619-624.

[Cosserat and Cosserat 1909] E. Cosserat and F. Cosserat, Théorie des corps déformables, Hermann, Paris, 1909.

[Dadgar-Rad and Beheshti 2017] F. Dadgar-Rad and A. Beheshti, "A nonlinear strain gradient finite element for microbeams and microframes”, Acta Mech. 228:5 (2017), 1941-1964.

[dell'Isola et al. 2016] F. dell'Isola, A. Della Corte, I. Giorgio, and D. Scerrato, "Pantographic 2D sheets: discussion of some numerical investigations and potential applications", Int. J. Nonlin. Mech. 80 (2016), 200-208.

[dell'Isola et al. 2019a] F. dell'Isola, P. Seppecher, J. J. Alibert, and et al., "Pantographic metamaterials: an example of mathematically driven design and of its technological challenges", Contin. Mech. Thermodyn. 31:4 (2019), 851-884.

[dell'Isola et al. 2019b] F. dell'Isola, P. Seppecher, M. Spagnuolo, E. Barchiesi, F. Hild, T. Lekszycki, I. Giorgio, L. Placidi, U. Andreaus, M. Cuomo, S. R. Eugster, A. Pfaff, K. Hoschke, R. Langkemper, E. Turco, R. Sarikaya, A. Misra, M. De Angelo, F. D’Annibale, A. Bouterf, X. Pinelli, A. Misra, B. Desmorat, M. Pawlikowski, C. Dupuy, D. Scerrato, P. Peyre, M. Laudato, L. Manzari, P. Göransson, C. Hesch, S. Hesch, P. Franciosi, J. Dirrenberger, F. Maurin, Z. Vangelatos, C. Grigoropoulos, V. Melissinaki, M. Farsari, W. Muller, B. E. Abali, C. Liebold, G. Ganzosch, P. Harrison, R. Drobnicki, L. Igumnov, F. Alzahrani, and T. Hayat, "Advances in pantographic structures: design, manufacturing, models, experiments and image analyses", Contin. Mech. Thermodyn. 31:4 (2019), $1231-1282$.

[Eringen 1999] A. C. Eringen, "Theory of micropolar elasticity”, Chapter 5, pp. 101-248 in Microcontinuum field theories, vol. I: Foundations and solids, Springer, 1999.

[Fleck et al. 1994] N. A. Fleck, G. M. Muller, M. F. Ashby, and J. W. Hutchinson, "Strain gradient plasticity: theory and experiment", Acta. Metall. Mater. 42:2 (1994), 475-487.

[Greco 2020] L. Greco, "An iso-parametric $\mathrm{G}^{1}$-conforming finite element for the nonlinear analysis of Kirchhoff rod, I: The 2D case”, Contin. Mech. Thermodyn. 32:5 (2020), 1473-1496.

[Greco and Cuomo 2014] L. Greco and M. Cuomo, "An implicit $G^{1}$ multi patch B-spline interpolation for Kirchhoff-Love space rod”, Comput. Methods Appl. Mech. Engrg. 269 (2014), 173-197.

[Greco et al. 2017] L. Greco, M. Cuomo, L. Contrafatto, and S. Gazzo, "An efficient blended mixed B-spline formulation for removing membrane locking in plane curved Kirchhoff rods", Comput. Methods Appl. Mech. Engrg. 324 (2017), 476-511.

[Green and Rivlin 1964] A. E. Green and R. S. Rivlin, "Multipolar continuum mechanics", Arch. Rational Mech. Anal. 17:2 (1964), 113-147.

[Hassanpour et al. 2010] P. A. Hassanpour, E. Esmailzadeh, W. L. Cleghorn, and J. K. Mills, "Nonlinear vibration of micromachined asymmetric resonators", J. Sound Vib. 329:13 (2010), 25472564. 
[Hu et al. 2004] Y. C. Hu, C. M. Chang, and S. C. Huang, "Some design considerations on the electrostatically actuated microstructures”, Sensor. Actuator. A Phys. 112:1 (2004), 155-161.

[Hughes et al. 2005] T. J. R. Hughes, J. A. Cottrell, and Y. Bazilevs, "Isogeometric analysis: CAD, finite elements, NURBS, exact geometry and mesh refinement", Comput. Methods Appl. Mech. Engrg. 194:39-41 (2005), 4135-4195.

[Kahrobaiyan et al. 2011] M. H. Kahrobaiyan, M. Asghari, M. Rahaeifard, and M. T. Ahmadian, "A nonlinear strain gradient beam formulation", Internat. J. Engrg. Sci. 49:11 (2011), 1256-1267.

[Khakalo and Niiranen 2018] S. Khakalo and J. Niiranen, "Form II of Mindlin's second strain gradient theory of elasticity with a simplification: for materials and structures from nano- to macroscales", Eur. J. Mech. A Solids 71 (2018), 292-319.

[Khakalo and Niiranen 2019] S. Khakalo and J. Niiranen, "Lattice structures as thermoelastic strain gradient metamaterials: evidence from full-field simulations and applications to functionally stepwise-graded beams", Compos. Part B Eng. 177 (2019), art. id. 107224.

[Khakalo and Niiranen 2020] S. Khakalo and J. Niiranen, "Anisotropic strain gradient thermoelasticity for cellular structures: plate models, homogenization and isogeometric analysis", J. Mech. Phys. Solids 134 (2020), art. id. 103728.

[Khakalo et al. 2018] S. Khakalo, V. Balobanov, and J. Niiranen, "Modelling size-dependent bending, buckling and vibrations of 2D triangular lattices by strain gradient elasticity models: applications to sandwich beams and auxetics", Internat. J. Engrg. Sci. 127 (2018), 33-52.

[Kiendl et al. 2015] J. Kiendl, F. Auricchio, T. J. R. Hughes, and A. Reali, "Single-variable formulations and isogeometric discretizations for shear deformable beams", Comput. Methods Appl. Mech. Engrg. 284 (2015), 988-1004.

[Kong et al. 2009] S. Kong, S. Zhou, Z. Nie, and K. Wang, "Static and dynamic analysis of micro beams based on strain gradient elasticity theory", Internat. J. Engrg. Sci. 47:4 (2009), 487-498.

[Lam et al. 2003] D. C. C. Lam, F. Yang, A. C. M. Chong, J. Wang, and P. Tonga, "Experiments and theory in strain gradient elasticity", J. Mech. Phys. Solids 51:8 (2003), 1477-1508.

[Lazar and Po 2015] M. Lazar and G. Po, "The non-singular Green tensor of Mindlin's anisotropic gradient elasticity with separable weak non-locality", Phys. Lett. A 379:24-25 (2015), 1538-1543.

[Lazopoulos 2004] K. A. Lazopoulos, "On the gradient strain elasticity theory of plates", Eur. J. Mech. A Solids 23:5 (2004), 843-852.

[Lazopoulos 2012] A. K. Lazopoulos, "Dynamic response of thin strain gradient elastic beams", Int. J. Mech. Sci. 58:1 (2012), 27-33.

[Lazopoulos and Lazopoulos 2010] K. A. Lazopoulos and A. K. Lazopoulos, "Bending and buckling of thin strain gradient elastic beams", Eur. J. Mech. A Solids 29:5 (2010), 837-843.

[Lazopoulos and Lazopoulos 2011] K. A. Lazopoulos and A. K. Lazopoulos, "Nonlinear strain gradient elastic thin shallow shells", Eur. J. Mech. A Solids 30:3 (2011), 286-292.

[Lazopoulos et al. 2014] A. K. Lazopoulos, K. A. Lazopoulos, and G. Palassopoulos, "Nonlinear bending and buckling for strain gradient elastic beams", Appl. Math. Model. 38:1 (2014), 253-262.

[Lun et al. 2006] F.-y. Lun, P. Zhang, F.-b. Gao, and H.-g. Jia, "Design and fabrication of microopto-mechanical vibration sensor”, Microfab. Tech. 2006:1 (2006), 61-64.

[Lurie and Solyaev 2018] S. Lurie and Y. Solyaev, "Revisiting bending theories of elastic gradient beams", Internat. J. Engrg. Sci. 126 (2018), 1-21.

[Luu et al. 2015] A.-T. Luu, N.-I. Kim, and J. Lee, "Bending and buckling of general laminated curved beams using NURBS-based isogeometric analysis", Eur. J. Mech. A Solids 54 (2015), 218231. 
[Ma et al. 2008] H. M. Ma, X.-L. Gao, and J. N. Reddy, "A microstructure-dependent Timoshenko beam model based on a modified couple stress theory", J. Mech. Phys. Solids 56:12 (2008), 33793391.

[Mindlin 1964] R. D. Mindlin, "Micro-structure in linear elasticity", Arch. Rational Mech. Anal. 16 (1964), 51-78.

[Mindlin and Eshel 1968] R. D. Mindlin and N. N. Eshel, "On first strain-gradient theories in linear elasticity”, Int. J. Solids Struct. 4:1 (1968), 109-124.

[Moghimi Zand and Ahmadian 2009] M. Moghimi Zand and M. T. Ahmadian, "Vibrational analysis of electrostatically actuated microstructures considering nonlinear effects", Commun. Nonlinear Sci. Numer. Sim. 14:4 (2009), 1664-1678.

[Niiranen et al. 2016] J. Niiranen, S. Khakalo, V. Balobanov, and A. H. Niemi, "Variational formulation and isogeometric analysis for fourth-order boundary value problems of gradient-elastic bar and plane strain/stress problems", Comput. Methods Appl. Mech. Engrg. 308 (2016), 182-211.

[Niiranen et al. 2017] J. Niiranen, J. Kiendl, A. H. Niemi, and A. Reali, "Isogeometric analysis for sixth-order boundary value problems of gradient-elastic Kirchhoff plates", Comput. Methods Appl. Mech. Engrg. 316 (2017), 328-348.

[Niiranen et al. 2019] J. Niiranen, V. Balobanov, J. Kiendl, and S. B. Hosseini, "Variational formulations, model comparisons and numerical methods for Euler-Bernoulli micro- and nano-beam models", Math. Mech. Solids 24:1 (2019), 312-335.

[Park and Gao 2006] S. K. Park and X.-L. Gao, "Bernoulli-Euler beam model based on a modified couple stress theory", J. Micromech. Microeng. 16:11 (2006), 2355-2359.

[Piegl and Tiller 1997] L. Piegl and W. Tiller, The NURBS book, 2nd ed., Springer, 1997.

[Ramezani 2012] S. Ramezani, "A micro scale geometrically non-linear Timoshenko beam model based on strain gradient elasticity theory”, Int. J. Nonlin. Mech. 47:8 (2012), 863-873.

[Ramezani 2013] S. Ramezani, "Nonlinear vibration analysis of micro-plates based on strain gradient elasticity theory", Nonlinear Dynam. 73:3 (2013), 1399-1421.

[Reinaldo Goncalves et al. 2016] B. Reinaldo Goncalves, J. Jelovica, and J. Romanoff, "A homogenization method for geometric nonlinear analysis of sandwich structures with initial imperfections", Int. J. Solids Struct. 87 (2016), 194-205.

[Rickert et al. 2019] W. Rickert, E. N. Vilchevskaya, and W. H. Müller, "A note on Couette flow of micropolar fluids according to Eringen's theory”, Math. Mech. Complex Syst. 7:1 (2019), 25-50.

[Stölken and Evans 1998] J. S. Stölken and A. G. Evans, "A microbend test method for measuring the plasticity length scale", Acta. Mater. 46:14 (1998), 5109-5115.

[Thai et al. 2014] C. H. Thai, S. Kulasegaram, L. V. Tran, and H. Nguyen-Xuan, "Generalized shear deformation theory for functionally graded isotropic and sandwich plates based on isogeometric approach", Comput. Struct. 141 (2014), 94-112.

[Thai et al. 2017] H.-T. Thai, T. P. Vo, T.-K. Nguyen, and S.-E. Kim, “A review of continuum mechanics models for size-dependent analysis of beams and plates", Compos. Scruct. 177 (2017), 196-219.

[Timoshenko and Goodier 1970] S. P. Timoshenko and J. N. Goodier, Theory of elastic stability, 3rd ed., McGraw-Hill, New York, 1970.

[Tran and Kim 2018] L. V. Tran and S.-E. Kim, "Stability analysis of multi-layered plates subjected to partial edge compression with and without initial imperfection", Compos. Scruct. 205 (2018), 26-41. 
[Tran et al. 2013] L. V. Tran, A. J. M. Ferreira, and H. Nguyen-Xuan, "Isogeometric analysis of functionally graded plates using higher-order shear deformation theory", Compos. Part B Eng. 51 (2013), 368-383.

[Tran et al. 2015] L. V. Tran, J. Lee, H. Nguyen-Van, H. Nguyen-Xuan, and M. A. Wahaba, "Geometrically nonlinear isogeometric analysis of laminated composite plates based on higher-order shear deformation theory", Int. J. Nonlin. Mech. 72 (2015), 42-52.

[Turner and Wiehn 2001] J. A. Turner and J. S. Wiehn, "Sensitivity of flexural and torsional vibration modes of atomic force microscope cantilevers to surface stiffness variations", Nanotechnology 12:3 (2001), 322-330.

[Vo and Nanakorn 2020] D. Vo and P. Nanakorn, "Geometrically nonlinear multi-patch isogeometric analysis of planar curved Euler-Bernoulli beams", Comput. Methods Appl. Mech. Engrg. 366 (2020), art. id. 113078.

[Wang et al. 2010] B. Wang, J. Zhao, and S. Zhou, "A micro scale Timoshenko beam model based on strain gradient elasticity theory", Eur. J. Mech. A Solids 29:4 (2010), 591-599.

[Xia et al. 2010] W. Xia, L. Wang, and L. Yin, "Nonlinear non-classical microscale beams: static bending, postbuckling and free vibration”, Internat. J. Engrg. Sci. 48:12 (2010), 2044-2053.

[Yang et al. 2002] F. Yang, A. C. M. Chong, D. C. C. Lam, and P. Tong, "Couple stress based strain gradient theory for elasticity”, Int. J. Solids Struct. 39:10 (2002), 2731-2743.

Received 21 Nov 2019. Revised 18 May 2020. Accepted 23 Jun 2020.

LOC V. TRAN: tranvinhloc@tdtu.edu.vn

Faculty of Civil Engineering, Ton Duc Thang University, Ho Chi Minh City, Vietnam

JARKKO NIIRANEN: jarkko.niiranen@aalto.fi

Department of Civil Engineering, Aalto University, Espoo, Finland 



\section{Guidelines for Authors}

Authors may submit manuscripts in PDF format on-line at the submission page.

Originality. Submission of a manuscript acknowledges that the manuscript is original and and is not, in whole or in part, published or under consideration for publication elsewhere. It is understood also that the manuscript will not be submitted elsewhere while under consideration for publication in this journal.

Language. Articles in MEMOCS are usually in English, but articles written in other languages are welcome.

Required items. A brief abstract of about 150 words or less must be included. It should be selfcontained and not make any reference to the bibliography. If the article is not in English, two versions of the abstract must be included, one in the language of the article and one in English. Also required are keywords and a Mathematics Subject Classification or a Physics and Astronomy Classification Scheme code for the article, and, for each author, postal address, affiliation (if appropriate), and email address if available. A home-page URL is optional.

Format. Authors are encouraged to use $\mathrm{IAT}_{\mathrm{E} X}$ and the standard amsart class, but submissions in other varieties of $\mathrm{T}_{\mathrm{E}} \mathrm{X}$, and exceptionally in other formats, are acceptable. Initial uploads should normally be in PDF format; after the refereeing process we will ask you to submit all source material.

References. Bibliographical references should be complete, including article titles and page ranges. All references in the bibliography should be cited in the text. The use of $\mathrm{BIBT}_{\mathrm{E}} \mathrm{X}$ is preferred but not required. Tags will be converted to the house format, however, for submission you may use the format of your choice. Links will be provided to all literature with known web locations and authors are encouraged to provide their own links in addition to those supplied in the editorial process.

Figures. Figures must be of publication quality. After acceptance, you will need to submit the original source files in vector graphics format for all diagrams in your manuscript: vector EPS or vector PDF files are the most useful.

Most drawing and graphing packages - Mathematica, Adobe Illustrator, Corel Draw, MATLAB, etc. - allow the user to save files in one of these formats. Make sure that what you are saving is vector graphics and not a bitmap. If you need help, please write to graphics@msp.org with as many details as you can about how your graphics were generated.

Bundle your figure files into a single archive (using zip, tar, rar or other format of your choice) and upload on the link you been provided at acceptance time. Each figure should be captioned and numbered so that it can float. Small figures occupying no more than three lines of vertical space can be kept in the text ("the curve looks like this:"). It is acceptable to submit a manuscript with all figures at the end, if their placement is specified in the text by means of comments such as "Place Figure 1 here". The same considerations apply to tables.

White Space. Forced line breaks or page breaks should not be inserted in the document. There is no point in your trying to optimize line and page breaks in the original manuscript. The manuscript will be reformatted to use the journal's preferred fonts and layout.

Proofs. Page proofs will be made available to authors (or to the designated corresponding author) at a Web site in PDF format. Failure to acknowledge the receipt of proofs or to return corrections within the requested deadline may cause publication to be postponed. 
Mathematics and Mechanics of Complex Systems vol. 8 no. 4

On a stochastic approach to model the double phosphorylation/dephosphorylation cycle

Alberto Maria Bersani, Alessandro Borri, Francesco Carravetta,

Gabriella Mavelli and Pasquale Palumbo

A new comprehensive approach for bone remodeling under medium and high mechanical load based on cellular activity

Daniel George, Rachele Allena, Céline Bourzac, Stéphane Pallu, Morad Bensidhoum, Hugues Portier and Yves Rémond

Models for drug release of gentamicin in a polylactic acid matrix

Anna S. Morozova, Elena N. Vilchevskaya, Wolfgang H. Müller and Nikolay M. Bessonov

Analytical mechanics allows novel vistas on mathematical epidemic dynamics modeling

\section{Paul Steinmann}

A geometrically nonlinear Euler-Bernoulli beam model within strain gradient elasticity with isogeometric analysis and lattice structure applications

Loc V. Tran and Jarkko Niiranen

MEMOCS is a journal of the International Research Center for the Mathematics and Mechanics of Complex Systems at the Università dell' Aquila, Italy.

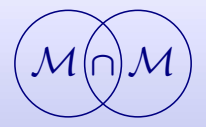

